TRANSACTIONS OF THE

AMERICAN MATHEMATICAL SOCIETY

Volume 351, Number 9 , Pages 3441-3497

S 0002-9947(99)02215-1

Article electronically published on April 27, 1999

\title{
NONLINEAR EQUATIONS AND WEIGHTED NORM INEQUALITIES
}

\author{
N. J. KALTON AND I. E. VERBITSKY
}

Abstract. We study connections between the problem of the existence of positive solutions for certain nonlinear equations and weighted norm inequalities. In particular, we obtain explicit criteria for the solvability of the Dirichlet problem

$$
\begin{gathered}
-\Delta u=v u^{q}+w, \quad u \geq 0 \quad \text { on } \Omega, \\
u=0 \text { on } \partial \Omega,
\end{gathered}
$$

on a regular domain $\Omega$ in $\mathbf{R}^{n}$ in the "superlinear case" $q>1$. The coefficients $v, w$ are arbitrary positive measurable functions (or measures) on $\Omega$. We also consider more general nonlinear differential and integral equations, and study the spaces of coefficients and solutions naturally associated with these problems, as well as the corresponding capacities.

Our characterizations of the existence of positive solutions take into account the interplay between $v, w$, and the corresponding Green's kernel. They are not only sufficient, but also necessary, and are established without any a priori regularity assumptions on $v$ and $w$; we also obtain sharp two-sided estimates of solutions up to the boundary. Some of our results are new even if $v \equiv 1$ and $\Omega$ is a ball or half-space.

The corresponding weighted norm inequalities are proved for integral operators with kernels satisfying a refined version of the so-called $3 G$-inequality by an elementary "integration by parts" argument. This also gives a new unified proof for some classical inequalities including the Carleson measure theorem for Poisson integrals and trace inequalities for Riesz potentials and Green potentials.

\section{INTRODUCTION}

The main goal of this paper is to obtain explicit criteria for the existence of positive solutions for a class of "superlinear" Dirichlet problems on a regular domain $\Omega \subset \mathbf{R}^{n}$. In particular we are interested in the solvability of the Dirichlet problem

$$
-\Delta u=v(x) u^{q}+w(x)
$$

on $\Omega$ where $n \geq 3$ subject to the conditions $u \geq 0$ and $u=0$ on $\partial \Omega$, when $q>1$ and $v, w$ are given positive measurable functions. By a solution of (1.1) we understand (more precise definitions are given below) a nonnegative measurable function $u$ satisfying a.e. on $\Omega$ the equivalent integral equation $u=G\left(v u^{q}\right)+G w$ which follows by applying the corresponding Green's potential $G=(-\Delta)^{-1}$ to both sides

Received by the editors March 1, 1997 and, in revised form, August 15, 1997.

1991 Mathematics Subject Classification. Primary 35J60, 42B25, 47H15; Secondary 31B15.

The first author was partially supported by NSF grant DMS-9500125, and the second by NSF grant DMS-9401493 and the University of Missouri Research Board grant RB-96029. 
of (1.1). It is also of interest to replace $v$ and $w$ by positive measures, so that the equation becomes

$$
-\Delta u=\sigma u^{q}+\omega
$$

where $\sigma$ and $\omega$ are arbitrary positive Borel measures on $\Omega$, and consider a more general boundary condition $u=\phi$ on $\partial \Omega$ for a nonnegative measurable function $\phi$; then solutions of (1.2) are understood in the analogous sense and are defined $\sigma$-a.e. on $\Omega$.

We observe that in the case $q=1$ these equations turn into the inhomogeneous Schrödinger equation with potentials $v$ and $\sigma$ respectively. Thus (1.1) and (1.2) may be referred to as the $q$-Schrödinger equations, and the techniques needed to treat them are reminiscent of those employed in [12], [13], [16], [17], [23], [35], [47], etc.

Equations of this type are widely used in differential geometry, physics, astronomy, and numerous applied problems with "nonlinear sources" (heat transfer, fluid flow, control theory, etc.; see [32] and the bibliography therein). They fall into the class of equations with convex nonlinearities which generally are known to be more difficult to investigate than equations with concave operators (see [29]). In a sense, (1.1) and (1.2) have become model problems for nonlinear analysts, especially after H. Brezis and L. Nirenberg studied in [8] the homogeneous problem related to (1.1) with $w \equiv 0$ and $v \equiv 1$ in the difficult "critical case" $q=(n+2) /(n-2)$.

The solvability of the inhomogeneous problems (1.1) and (1.2) with variable coefficients has been studied extensively, mostly under strong additional assumptions on the potentials and data. We mention sufficient conditions of solvability for a general class of boundary value problems with unbounded coefficients and domains $\Omega$ established by M. Schechter (see [47]). However, the necessary conditions for solvability (criteria of "nonsolvabilty") which match sufficiency results are more difficult to obtain. This problem is solved in the present paper for a wide class of nonlinear differential and integral equations.

A starting point for us in studying (1.2) was the following sharp criterion for the existence of solutions in the case $\sigma \equiv 1$ and $\omega$ compactly supported in $\Omega$ due to D. R. Adams and M. Pierre [3]. (Note that as explained below the "critical index" for this problem is $q=n /(n-2)$.)

Theorem 1.1 [3]. Suppose that $1<q<\infty, \sigma \equiv 1$, and $\omega$ is a compactly supported measure on a bounded regular domain $\Omega$.

(1) If there exists a solution for (1.2) which vanishes at the boundary, then

$$
|E|_{\omega} \leq C \operatorname{Cap}_{p}(E), \quad E \Subset \Omega,
$$

where $C$ is independent of compact sets $E$ and $\operatorname{Cap}_{p}(\cdot)$ is the capacity associated with the Sobolev space $W^{2, p}\left(\mathbf{R}^{n}\right), 1 / p+1 / q=1$.

(2) Conversely, (1.2) has a solution with zero boundary values if (1.3) holds with a small enough constant $C<C(q, n, \omega, \Omega)$.

We observe that Theorem 1.1 provides not only sufficient but also necessary conditions for solvability in explicit geometric terms. The classes of measures characterized by estimates of type (1.3) are well studied in potential theory starting from the work of V. G. Maz'ya [34] on spectral problems for Schrödinger operators in the early sixties. (We cite the books [2], [24], [35], and [57] as an invaluable source on capacities, potential theory, related function spaces and applications to 
partial differential equations.) There also are equivalent alternative characterizations of these classes which do not involve capacities and play an important role in the sequel (see [27], [37], [44], [45], [46], [52]).

Note that if $1<q<n /(n-2)$, then $\inf _{E} \operatorname{Cap}_{p}(E)>0$ for all $E \subset \operatorname{supp} \omega$ so that $q=n /(n-2)$ is a critical index. In the more interesting case $q \geq n /(n-2)$ however, for compactly supported $\omega$, one deals essentially with $\Omega=\mathbf{R}^{n}$ where the capacities $\operatorname{Cap}_{p}(\cdot)$ are applicable. They are not adequate for the analysis of solutions and coefficients up to the boundary.

The study of the boundary behavior for nonlinear problems of this type requires new methods. Even in the case $\sigma \equiv 1$ on a bounded domain with smooth boundary the solvability problem for (1.2) was open for noncompactly supported $\omega$. A solution to this and more general problems in both capacitary and non-capacitary form is given below.

We remark that similar questions for the Dirichlet problem

$$
\left\{\begin{array}{l}
\Delta u=\sigma u^{q}+\omega, \quad u \geq 0 \quad \text { on } \Omega \\
u=\phi \quad \text { on } \partial \Omega
\end{array}\right.
$$

with $\Delta$ in place of $-\Delta$ are in the center of the current work of probabilists. They consider (1.4) in the case $\sigma \equiv 1$ to study the so-called superdiffusions (see [30], [14], [15]) for $1<q \leq 2$. Unfortunately, at the moment no probabilistic models seem to be known for (1.2), or (1.4) with $q>2$. Criteria of solvability for (1.2) and (1.4), at least in the well studied case $\sigma \equiv 1$ and $\operatorname{supp} \omega \Subset \Omega$, are known to be different: solutions for (1.4) exist under much weaker assumptions (absolute continuity of $\omega$ with respect to the corresponding capacity) than for (1.2).

In order to study the problems stated above we first develop a general technique for studying a class of nonlinear operator equations. We then consider certain nonlinear integral equations related to (1.1), (1.2), or more general differential equations via Green's functions and the corresponding potential theory. This approach which is applicable to many other similar problems is developed in Sections 2-5.

Before discussing our approach in detail we would like to make some general comments. We do not use any variational theory, weighted Sobolev spaces, CalderonZygmund decompositions, or maximal function inequalities. In this respect our approach resembles some ideas of the original proof of T. Wolff's inequality which appeared in the context of the spectral synthesis problem for Sobolev spaces in [23]. (Note that the subsequent alternative proofs of Wolff's inequality due to Per Nilsson, J. L. Lewis, and D. R. Adams, see [2], p. 126, are not enough for our purposes.) We develop a new discrete decomposition for integral operators with respect to an arbitrary measure. This leads to sharp estimates of the nonlinear iterations of Green's potentials with precise estimates of the constants involved which is most important in this paper.

We also would like to point out interesting connections (in the easier part which involves $L^{p}$-estimates for integral operators) with the Hilbert space proof of the Carleson measure theorem due to S. A. Vinogradov (see [40]) and its weighted analogue used recently by S. Treil and A. Volberg [50] in the vector valued version of the Hunt-Muckenhoupt-Wheeden theorem in case $p=2$.

However, we avoid using dyadic decompositions, sophisticated capacitary inequalities, or any known tests for boundedness of integral operators or quadratic forms. Our proofs here are based on a quite elementary argument which resembles the proof of Hardy's inequality and works for all $1<p<\infty$. In particular it 
yields a simple proof of some deep classical results (Carleson measure inequality [10], trace inequalities of Maz'ya-D. Adams-Dahlberg [2], [35]) and their generalizations. Another application of these ideas to the problem of $\Lambda_{p}$ sets for Legendre and Jacobi polynomials is given in [26] where the underlying space is assumed to be discrete. Similar inequalities also appear in the problem of solvability for multidimensional Riccati's equations [22], spectral estimates for Schrödinger operators and multipliers of Sobolev spaces [37], etc.

We now turn to a more detailed discussion of our main results. Let $X$ be a metric space and suppose $\sigma$ is a fixed positive sigma-finite Borel measure on $X$. Suppose $K: X \times X \rightarrow[0,+\infty]$ is a Borel kernel function. We write

$$
K f(x)=K(f d \sigma)(x)=\int_{X} K(x, y) f(y) d \sigma(y)
$$

for any measurable function $f \geq 0$. Occasionally we write $K^{\sigma} f$ rather than $K f$ to emphasize the role of the underlying measure $\sigma$. In particular, if $f \equiv 1$, we have $K \mathbf{1}=K^{\sigma} \mathbf{1}=K \sigma$. Similarly, for any Borel measure $\omega$ on $X$, we set

$$
K \omega(x)=K^{\omega} \mathbf{1}(x)=\int_{X} K(x, y) d \omega(y),
$$

which hopefully does not lead to any confusion.

The problems (1.1), (1.2) and many similar problems can be transformed into "superlinear" integral equation of the type

$$
u(x)=K u^{q}(x)+f(x) \quad(\sigma \text {-a.e. })
$$

where $f \geq 0$ is given and we require a nonnegative solution. Let us denote by $S_{q, K}$ the set of all $f$ such that (1.5) has a solution (i.e. there is a measurable $u \geq 0$ satisfying (1.5)). This problem has been considered in several places in the literature (cf. [3], [6], [29], [32], [52] ). In the examples we wish to consider, the kernel $K$ has an additional property which we term the quasi-metric assumption i.e. $K$ is symmetric, $K(x, y)>0$ for all $x, y$, and there is a constant $\kappa$ such that for $x, y, z \in X$ we have

$$
\frac{1}{K(x, y)} \leq \kappa\left(\frac{1}{K(x, z)}+\frac{1}{K(z, y)}\right) .
$$

Then we can introduce a quasi-metric structure via $\rho(x, y)=K(x, y)^{-1}$. Note however that we do not require $\rho(x, x)=0$. If we define the $\rho$-ball $B_{a}(x)=\{y$ : $\rho(x, y) \leq a\}$, then we can write

$$
K^{\sigma} f(x)=K \nu(x)=\int_{0}^{\infty} \frac{\left|B_{t}(x)\right|_{\nu}}{t^{2}} d t
$$

where $d \nu=f d \sigma, f \geq 0$.

We remark that in our main results we are able to avoid the usual assumption of the theory of homogeneous spaces in the sense of Coifman and Weiss [11] that there exists a doubling measure with respect to $\rho$ on $X$. Under that assumption our results are applicable to equations with generalized fractional integral operators of $[20]$.

Returning to (1.5) we seek to characterize those functions $f$ so that for some $\epsilon>0$ we have $\epsilon f \in S_{q, K}$ i.e. there is a solution to the equation

$$
u=K u^{q}+\epsilon f .
$$


In this context it is natural to introduce the solution space $\mathcal{Z}_{q, K}$ of all measurable functions $f$ such that for some $\epsilon>0$ we have $\epsilon|f| \in S_{q, K}$. If we have the quasimetric condition (1.6), then $\mathcal{Z}_{q, K}$ either reduces to $\{0\}$ or is a Banach function space on $(X, \sigma)$ with associated norm $\|f\|_{\mathcal{Z}}=\inf \left\{\lambda>0: f \in \lambda S_{q, K}\right\}$, and all solutions of the equation belong to $\mathcal{Z}_{q, K}$.

In Section 2, which consists mainly of background material, we develop a general theory of the solution space $\mathcal{Z}=\mathcal{Z}_{q, T}$ associated to the equation $u=T u^{q}+f$ where $T$ is an arbitrary positive operator on the space of measurable functions. Most results on nonlinear operator equations of this type we have found in the literature (see e.g. [29], [32]) contain only sufficient conditions for solvability in particular function spaces which are not intrinsically related to the equation. To bridge this gap, we use techniques borrowed from the Banach lattice theory and in particular some facts from the Nikishin-Maurey theory (see [31], [33], [39]) to identify $\mathcal{Z}_{q, T}$ as a Banach function space naturally associated with the problem.

Under mild assumptions, we demonstrate a number of different characterizations of this space. For example we show that $\mathcal{Z}$ is invariant under the mapping $f \rightarrow$ $\mathcal{A} f=T f^{q}$ and that $0 \leq f \in \mathcal{Z}$ if and only if

$$
\limsup _{n \rightarrow \infty}\left(\mathcal{A}^{n} f\right)^{1 / q^{n}} \in L^{\infty}(\sigma) .
$$

We also show that earlier results of P. Baras and M. Pierre [6] can be recast as identifying the predual space of $\mathcal{Z}$. The Köthe dual $\mathcal{Z}^{\prime}$ of $\mathcal{Z}$ is shown to be the predual and its norm is given by the formula:

$$
\|g\|_{\mathcal{Z}^{\prime}}=p q^{p-1} \inf \left\{\int \frac{h^{p}}{\left(T^{*} h\right)^{p-1}} d \sigma: h \geq|g|\right\} .
$$

Here $T^{*}$ is the adjoint of the operator $T, 1 / p+1 / q=1$, and the equation $u=\mathcal{A} u+f$ is solvable if and only if $f \geq 0$ lies in the unit ball of $\mathcal{Z}$. This is quite similar to a result proved by Baras and Pierre, who consider more general convex functions than $x \rightarrow x^{q}$. It should be noted that our result applies to general positive operators (not simply operators defined by kernels) and that our method of proof is quite different from that of Baras and Pierre, depending only on quite simple duality arguments. Although this characterization of the predual space $\mathcal{Z}^{\prime}$ seems to us both important and interesting, it is not used to establish the main results of the paper.

At the same time in Section 2 we introduce the Banach function space $\mathcal{W}_{p, T}$ of $L^{p}$-weights associated with the corresponding weighted norm inequalities for future use. Its $q$-convexification which contains $\mathcal{Z}_{q, T}$ plays an important role in the sequel and is intimately related to the general theory of $L^{p}$-capacities developed by N. G. Meyers (see [2]).

In Section 3, we carry out the study of the solution space further for the case of an operator defined by a kernel $K$ satisfying the quasi-metric assumption. Our main technical tool here is a decomposition of the operator into its upper and lower parts, the latter being almost constant on quasi-metric balls (Proposition 3.4). We show that if $\omega$ is a $\sigma$-finite Borel measure on $X$ and

$$
K \omega(x)=K^{\omega} \mathbf{1}(x)=\int K(x, y) d \omega(y)
$$


then $K \omega \in \mathcal{Z}$ implies the inequality:

$$
\sup _{\substack{x \in X \\ a>0}}\left(\int_{0}^{a} \frac{\left|B_{t}(x)\right|_{\sigma}}{t^{2}} d t\right)\left(\int_{a}^{\infty} \frac{\left|B_{t}(x)\right|_{\omega}}{t^{2}} d t\right)^{q / p}<\infty
$$

which reflects the interplay between the local regularity of $\sigma$ and the behavior of the "tails" of $K \omega$. It is reminiscent of the Kato class for the potential $\sigma$ as $q \rightarrow 1$ (see [13]). We call (1.9) the infinitesimal inequality because of the method of the proof which boils down to careful pointwise estimates of $\left(\mathcal{A}^{n} f\right)^{1 / q^{n}}$ as $n \rightarrow \infty$; they involve sharp constants depending on $q$ and $\kappa$. In particular if $f \in \mathcal{Z}$, then the infinitesimal inequality holds for the measure $d \omega=f^{q} d \sigma$.

In Section 4, we then turn to the relationship between solutions of (1.2) and weighted norm inequalities of the type:

$$
\int\left(K^{\sigma} g\right)^{p} d \omega \leq C \int g^{p} d \sigma
$$

whenever $g \geq 0$ and $g \in L^{p}(\sigma), 1 / p+1 / q=1$. We prove that if $K \omega \in \mathcal{Z}$, then the weighted norm inequality (1.10) holds, and further that (1.10) is also implied by the inequality

$$
\int\left(K^{\sigma} g\right)^{p}(K \omega)^{q} d \sigma \leq C \int g^{p} d \sigma .
$$

In general $K \omega \in \mathcal{Z}$ is equivalent to a sequence of weighted norm inequalities

$$
\int\left(K^{\sigma} g\right)^{p} d \omega_{j} \leq C_{j} \int g^{p} d \sigma, \quad j=1,2, \ldots,
$$

where $d \omega_{0}=d \omega$ and $d \omega_{j+1}=\left(K \omega_{j}\right)^{q} d \sigma$, with a good control of the imbedding constants: one should require $\limsup _{j \rightarrow \infty} C_{j}^{1 / q^{j}}<\infty$ (Theorem 4.7); however this result is a little unwieldy.

Our main result of the section (Theorems 4.8 and 4.9 ) is that $K \omega \in \mathcal{Z}$ if and only if both the infinitesimal inequality (1.9) and the weighted norm inequality (1.10) hold, which is also equivalent to the pointwise inequality $K(K \omega)^{q} \leq C K \omega$. Also in these results it is possible to replace (1.10) by its weak-type analogue, or even the well-known testing condition of Sawyer type (see [44], [45], [46])

$$
\int_{B}\left(K^{\omega} \chi_{B}\right)^{q} d \sigma \leq C|B|_{\omega}
$$

for all quasi-metric balls $B=B_{a}(x)$. This leads to the following theorem.

Theorem 1.2. Let $\omega, \sigma \in \mathcal{M}_{+}(X)$ and let $K f=K^{\sigma} f$. Then the following are equivalent:

(1) $K \omega \in \mathcal{Z}_{q, K}$ i.e. the equation $u=K u^{q}+\epsilon K \omega$ has a solution for some $\epsilon>0$.

(2) $\omega$ satisfies both the infinitesimal inequality (1.9) and weighted norm inequality (1.10).

(3) $\omega$ satisfies both the infinitesimal inequality (1.9) and testing inequality (1.11).

(4) There exists a constant $C$ so that $K(K \omega)^{q} \leq C K \omega<\infty \sigma$-a.e.

It is easy to see that if in (4) the constant $C \leq q^{-1} p^{1-q}$, then the equation $u=K u^{q}+K \omega$ has a solution $u$ so that $K \omega \leq u \leq p K \omega$. This remark means that Theorem 1.2 makes it possible to characterize the existence of positive solutions in $L^{r}$ spaces (or any other ideal space).

Similar results hold for all $0 \leq f \in \mathcal{Z}$. 
Theorem 1.3. Let $0 \leq f<\infty \sigma$-a.e. and $d \omega=f^{q} d \sigma$. Then the equation $u=$ $K u^{q}+\epsilon f$ has a solution for some $\epsilon>0$ if and only if any one of statements (2)-(4) of Theorem 1.2 holds.

It follows from the general estimates of Section 2 that if $d \omega=f^{q} d \sigma$ and $K(K \omega)^{q} \leq q^{-q} p^{q(1-q)} K \omega<\infty$ as in statement (4) of Theorem 1.3, then the equation $u=K u^{q}+f$ has a solution so that $f+K f^{q} \leq u \leq f+p^{q} K f^{q}$.

Note that in the proof of Theorems 1.2 and 1.3 we do not use any known two weight theorems (cf. [44], [45]); our proofs are self-contained and shed new light on the role of weighted norm inequalities and testing conditions of Sawyer type.

In Section 5 we introduce notions of capacity associated to the kernel and then study conditions on the kernel $K$ and the measure $\sigma$ under which the statement $K \omega \in \mathcal{Z}$ becomes equivalent to the weighted norm inequality (1.10). This requires that (1.10) implies (1.9). In fact we give two theorems of this type. In Theorems 5.6 and 5.9 we give conditions on the kernel $K$ so that $K \omega \in \mathcal{Z}$ if (1.10) holds or if a weaker capacitary condition holds:

$$
|E|_{\omega} \leq C \text { Cap } E
$$

where

$$
\text { Cap } E=\inf \left\{\int g^{p} d \sigma: 0 \leq g, K g \geq \chi_{E}\right\} .
$$

It is also possible to replace this condition by a Sawyer-type testing condition. The hypothesis of Theorem 5.6 (which can be shown to be necessary for the conclusion under certain mild assumptions on $K$ ) is that for some constant $C$ and every $x \in X$ and $a>0$ we have

$$
\int_{0}^{a} \frac{\left|B_{t}(x)\right|_{\sigma}}{t^{2}} d t \leq C a^{q-1} \int_{a}^{\infty} \frac{\left|B_{t}(x)\right|_{\sigma}}{t^{1+q}} d t<\infty .
$$

Roughly speaking this condition implies that the behavior of the kernel at infinity dominates the behavior locally. The hypothesis of Theorem 5.9 replaces this by the assumption that for some $C$ and every $x \in X, a>0$ we have both:

$$
\int_{0}^{2 a} \frac{\left|B_{t}(x)\right|_{\sigma}}{t^{2}} d t \leq C \int_{0}^{a} \frac{\left|B_{t}(x)\right|_{\sigma}}{t^{2}} d t
$$

and

$$
\sup _{y \in B_{a}(x)} \int_{0}^{a} \frac{\left|B_{t}(y)\right|_{\sigma}}{t^{2}} d t \leq C \int_{0}^{a} \frac{\left|B_{t}(x)\right|_{\sigma}}{t^{2}} d t .
$$

Conditions (1.14) and (1.15) essentially are assumptions that measure $\sigma$ is close to being invariant for the kernel $K$. For convenience we state these results as the following theorem.

Theorem 1.4. Let $K$ be a quasi-metric kernel. Assume that there is a constant $C$ so that for every $x \in X$ and $a>0$, either (1.13), or both (1.14) and (1.15) hold. Then $\mathcal{Z}_{q, K} \neq\{0\}$ and the following statements are equivalent:

(1) $\omega$ satisfies the weighted norm inequality (1.10).

(2) $\omega$ satisfies the capacity condition $|E|_{\omega} \leq$ Cap E for all Borel sets E. (Equivalently the weak-type analogue of (1.10) holds.)

(3) $\omega$ satisfies the testing condition (1.11).

(4) $K \omega \in \mathcal{Z}_{q, K}$ i.e. for some $\epsilon>0$ there is a solution $u$ of the equation $u=$ $K u^{q}+\epsilon K \omega$. 
(5) There is a constant $C$ so that $K(K \omega)^{q} \leq C K \omega$.

The proof of Theorem 1.4 involves a quantity which under mild assumptions is equivalent to the capacity of a ball. The two-sided estimates of Cap $B$ are obtained in Theorem 5.4 without any restrictions on the underlying measure $\sigma$ for a wide class of $K$. (This is a generalization of D. Adams's formula for the weighted capacity of a ball proved in [1] in the case of Riesz potentials for $\sigma \in A_{\infty}$; an upper estimate for arbitrary $\sigma$ can be found in [51].) This should be compared to a similar estimate of $\left\|\chi_{B}\right\|_{\mathcal{Z}_{q, K}}$ in Section 4 which clarifies the role of the infinitesimal inequality.

In Sections 6 and 7 we give some applications of our ideas to concrete problems of the type introduced earlier. Section 6 is devoted to convolution operators on $\mathbf{R}^{n}$. In particular we translate our results for the Riesz potential of order $\alpha$ i.e. $I_{\alpha}=(-\Delta)^{-\alpha / 2}$. The results developed in Sections 3, 4 and 5 can be translated directly to this setting taking into account the change between ordinary Euclidean distance and the quasi-distance induced by the quasi-metric $\rho$ (see Theorem 6.2 below). We also apply our results to the Poisson kernel to derive an extension of the recent result of Treil and Volberg [50] mentioned above.

In Section 7 we return to the problem which motivated this research, i.e. the equations (1.1) and (1.2). We consider more general differential operators and inhomogeneous boundary conditions. If $G$ denotes the Green's kernel for the Laplacian $\Delta$ on $\Omega$, then (1.1) can be transformed to the equation:

$$
u=G\left(v u^{q}\right)+G w .
$$

If we let $d \sigma=v(x) d x$, then we can consider this equation as being in the form of (1.5). It is easy to see that the Green's kernel $G$ fails to satisfy the quasi-metric assumption (1.6) in general, even for the simplest domains (e.g. the Euclidean ball or the half-space). However $G$ does satisfy the so-called $3 G$-inequality (see [12]) i.e.

$$
\frac{G(x, y) G(y, z)}{G(x, z)} \leq C\left(|x-y|^{2-n}+|y-z|^{2-n}\right) .
$$

In [7], Theorem 3.6 it is claimed that for Lipschitz domains one can replace the right-hand side by $G(x, y)+G(y, z)$ which would establish (1.6) for the Green's kernel but there is an error in the proof.

However if the boundary $\partial \Omega$ is smooth enough, the problem can be transformed to meet the condition (1.6). If $\partial \Omega$ is $C^{1,1}$, then the Naïm kernel (introduced in an equivalent form by Linda Naïm [38] in the theory related to Martin's kernels) is defined by

$$
N(x, y)=\frac{G(x, y)}{\delta(x) \delta(y)}
$$

where $\delta(x)=d(x, \partial \Omega)$ is the distance to the boundary. We show that $N$ does indeed satisfy (1.6), and this enables us to transform (1.16) to an equation for which our general theory is applicable. We observe that this inequality is sharper than (1.17) and gives the right estimates of the Green's kernel at the boundary.

These methods can be applied to more general second-order non-divergence uniformly elliptic differential operators $L$ with regular (bounded Hölder-continuous) coefficients in place of the Laplacian. Under these assumptions, it follows from the 
well-known estimates of the Green's kernels (see [55], [56], [5], [25], and the discussion in Section 7) that the corresponding Naïm kernel given by (1.18) satisfies condition (1.6).

By using this method we are able to give very general results on the solvability of the equations (1.1) and (1.2) as well as a characterization of trace inequalities for Green's potentials. In Theorem 7.5 we show that the equation

$$
-\Delta u=\sigma u^{q}+\epsilon \omega
$$

with $u \geq 0$ and $u=0$ on $\partial \Omega$ has a solution for some $\epsilon>0$ if and only if for some constant $C$ we have

$$
G\left[\sigma(G \omega)^{q}\right] \leq C G \omega,
$$

where $G \omega(x)=\int_{\Omega} G(x, y) d \omega(y)$. Here $\sigma$ and $\omega$ are arbitrary nonnegative measurable functions (or positive measures) on $\Omega$. We also give equivalent formulations in geometric terms involving the infinitesimal inequality and the testing inequality quoted above.

Finally we return to the Adams-Pierre theorem (Theorem 1.1) and show that our methods in this situation give a complete solution (up to a constant) to the problem of the existence of positive solutions. This corresponds to the case $\sigma \equiv 1$, but similar results are proved for arbitrary $\sigma$. We can then apply Theorem 1.4 and show that the solvability of (1.19) can be characterized in terms of a capacitary condition. We are thus able to remove the assumption that $\omega$ is compactly supported in $\Omega$.

Let

$$
\text { Cap } E=\inf \left\{\int_{\Omega} g^{p} \delta(x)^{1-p} d x: G g(x) \geq \delta(x) \chi_{E}(x), g \geq 0\right\}
$$

for any set $E \subset \Omega$.

Theorem 1.5. Let $\omega \in \mathcal{M}_{+}(\Omega)$ and $\sigma \equiv 1$. Then the Dirichlet problem (1.19) has a solution for some $\epsilon>0$ if and only if there is a constant $C$ so that

$$
\int_{E} \delta(x) d \omega(x) \leq C \text { Cap } E
$$

for every compact set $E$.

Moreover, (1.21) is equivalent to the pointwise condition (1.20).

As was mentioned above, if in (1.20) the constant $C \leq q^{-1} p^{1-q}$, then for $0<$ $\epsilon \leq 1$ (1.19) has a solution $u$ so that $G \omega \leq u \leq p G \omega$.

In the case when $\omega$ is compactly supported, the capacitary characterization of Theorem 1.5 reduces to the Adams-Pierre theorem since the capacity defined above can be then shown equivalent to the nonlinear Newtonian capacity associated with the Sobolev space $W^{2, p}\left(\mathbf{R}^{n}\right)$ used in [3].

After this paper was finished, we learned that H. Brezis and X. Cabre [9] considered very recently another special case of (1.19) where the inhomogeneous term $\omega$ is a positive uniformly bounded function on $\Omega .{ }^{1}$ In particular, they proved in a different way the following "nonsolvability" result (for bounded domains $\Omega$ with smooth boundary): (1.19) with bounded $\omega$ has no solutions unless $G\left(\delta^{q} \sigma\right) \in L^{\infty}(\Omega)$. Note

\footnotetext{
${ }^{1}$ See also the Addendum at the end of this paper.
} 
that in this case $G \omega(x) \asymp \delta(x)$, and our characterization (1.20) boils down to a sharper necessary and sufficient condition

$$
G\left(\delta^{q} \sigma\right)(x) \leq C \delta(x), \quad x \in \Omega .
$$

By Theorem 7.5 (see Sec. 7), it follows that (1.22) also characterizes completely the solvability of (1.19) with $\omega \in L^{\infty}(\Omega)$ for more general uniformly elliptic second order differential operators in place of the Laplacian.

All the results in Section 7 apply to much more general situations as explained therein. For instance, the only property of the differential operator with Green's function $G$ which is important for us is the fact that the kernel

$$
N(x, y)=s(x) G(x, y) s(y)^{-1}
$$

has the quasi-metric property for some weight function $s>0$; then the pointwise condition (1.20) which is invariant under this transformation of the kernel characterizes the solvability of the corresponding Dirichlet problem. We conjecture that this holds true for a wide class of differential operators with bounded measurable coefficients and non-smooth domains $\Omega$. There are many other potential applications of these ideas which we plan to explore in future work.

We would like to express our thanks to our colleague Zhongxin Zhao for his helpful comments concerning the $3 G$-inequalities.

\section{Superlinear PROBlems AND RELATED FUnCtion SPACES}

In this section we will introduce certain Banach function spaces which will play an important role in the later sections of the paper. We will also give an alternative approach to some results of Baras and Pierre [6] on the solvability of the "superlinear" problem $u=T u^{q}+f$ discussed in the introduction.

Let $X$ be a metric space and suppose $\sigma$ is a $\sigma$-finite measure on $X$. We denote by $L^{0}(\sigma)$ the space of all (equivalence classes) of real-valued Borel functions on $X$. The topology of $L^{0}(\sigma)$ is the usual topology of convergence in measure on sets of finite measure. We use $L_{+}^{0}$ to denote the positive cone $\left\{f: f \in L^{0}, f \geq 0\right\}$.

Let us first state two fundamental results which will be used in the sequel:

Theorem 2.1. Let $H$ be a closed bounded convex subset of $L_{+}^{0}$. Then:

(1) (Nikishin [39]) There exists a weight function $w \in L_{+}^{0}$ with $w>0$ a.e. such that $\sup _{f \in H} \int f w d \sigma<\infty$.

(2) (Komlos [28]) If $\left(f_{n}\right)$ is a sequence in $H$, then $\left(f_{n}\right)$ has a subsequence $\left(g_{n}\right)$ such that $\frac{1}{n}\left(g_{1}+\cdots+g_{n}\right)$ is a.e. convergent.

Nikishin's result is [39], Theorem 4, or see Maurey [33], Théorème 13. We observe that Komlos's result is usually stated for bounded sequences in $L^{1}$ but our statement follows from the usual Komlos theorem in view of (1). Also note that the conclusion of Komlos's theorem is much stronger than we have stated here: one can ensure that every subsequence of $\left(g_{n}\right)$ is Cesàro convergent a.e. to some fixed $h$. The version we will use can be established by much more simple means. In fact we do not need Cesàro means; any suitable sequence of convex combinations suffices.

We say that a convex subset $H$ of $L_{+}^{0}$ is solid if $f \in H$ and $0 \leq g \leq f$ a.e. implies that $g \in H$. We will also say that $H$ is nondegenerate if there exists $f \in H$ with $f>0$ a.e. Our next two results are surely well-known to specialists but we know of no reference where they are established in exactly this form. 
Proposition 2.2. Let $H$ be a solid convex subset of $L_{+}^{0}$. Then $H$ is closed if and only if whenever $f_{n} \uparrow f$ a.e. with $f_{n} \in H$ for all $n$, then $f \in H$.

Proof. Suppose $h_{n} \in H$ and $h_{n} \rightarrow f$ a.e. Then by applying Egoroff's theorem we can find an increasing sequence of Borel subsets $E_{m}$ of $X$ so that $h_{n} \rightarrow f$ uniformly on each $E_{m}, f \geq m^{-1}$ on $E_{m}$ and $\bigcup E_{m}=\operatorname{supp} f$. Now for fixed $m$ we can find $\epsilon_{n} \downarrow 0$ so that $\left(1-\epsilon_{n}\right) f \leq h_{n} \leq f$ on $E_{m}$. It follows that each $\left(1-\epsilon_{n}\right) f \chi_{E_{m}} \in H$ and hence we can apply the hypothesis twice to obtain $f \in H$.

If $H$ is a solid closed bounded convex subset of $L_{+}^{0}$, then we can define a Banach function space $\mathcal{X}=\mathcal{X}_{H}$ associated to $H$. Precisely if $f \in L^{0}$, we set

$$
\|f\|_{\mathcal{X}}=\inf \{\lambda:|f| \in \lambda C\}
$$

and then $\mathcal{X}=\left\{f:\|f\|_{\mathcal{X}}<\infty\right\}$. It is easily checked that $\mathcal{X}$ is a Banach lattice continuously embedded into $L^{0}$. Notice that the norm \|\|$_{\mathcal{X}}$ has the Fatou property i.e. $0 \leq f_{n} \uparrow f$ implies $0 \leq\left\|f_{n}\right\|_{X} \uparrow\|f\|_{\mathcal{X}}$. We shall say that $\mathcal{X}$ is nondegenerate if it contains a strictly positive function: this is easily seen to be equivalent to nondegeneracy of $H$.

For any convex subset $H$ of $L_{+}^{0}$ we can define

$$
H^{\prime}=\left\{g \in L_{+}^{0}: \int f g d \sigma \leq 1, \forall f \in H\right\} .
$$

It is clear that $H^{\prime}$ is closed solid and convex. If $H$ is bounded, then by Nikishin's Theorem 2.1 (1) $H^{\prime}$ is nondegenerate; if $H$ is nondegenerate, then $H^{\prime}$ is bounded. When $H$ is nondegenerate bounded convex and solid, then $\mathcal{X}_{H^{\prime}}$ is Köthe dual space

$$
\mathcal{X}^{\prime}=\left\{f: \int|f||g| d \sigma<\infty \quad \forall g \in \mathcal{X}\right\}
$$

of $\mathcal{X}$, equipped with the dual norm. The following observation is important for later applications so we display it as a lemma:

Lemma 2.3. If $H$ is a nondegenerate bounded closed solid convex set, then $H=$ $H^{\prime \prime}$ and $\mathcal{X}=\mathcal{X}^{\prime \prime}$ with equality of norms.

Proof. Using Nikishin's theorem again there exists $u \in H^{\prime}$ with $u>0$ a.e. Then $H$ is closed in $L^{1}(u d \sigma)$ or equivalently $u H$ is closed in $L^{1}$. Hence

$$
u H=\left\{f \geq 0: \int f g \leq 1 \forall 0 \leq g \in u^{-1} H^{\prime} \cap L^{\infty}\right\}
$$

and the lemma follows.

Next we define a generalized notion of a positive operator. Let $L_{+}^{0, \#}$ denote the space of (equivalence classes of) measurable functions $f: X \rightarrow[0, \infty]$. We define a positive operator to be a map $T: L_{+}^{0, \#} \rightarrow L_{+}^{0, \#}$ such that:

(P1) $T(\alpha f+\beta g)=\alpha T f+\beta T g$ if $\alpha, \beta \geq 0$, and $f, g \in L_{+}^{0, \#}$.

(P2) If $0 \leq f_{n} \uparrow f$ a.e., then $T f_{n} \uparrow T f$ a.e.

(P3) There exists $f$ with $f>0$ a.e. such that $T f<\infty$ a.e.

We say that $T$ is strictly positive if in addition $T f=0$ a.e. implies $f=0$ a.e.

In all our applications $T$ will be given by a kernel function $K$. That is, we will be given a nonnegative Borel function $K$ on $X \times X$ and then

$$
T f(x)=K f(x)=\int K(x, y) f(y) d \sigma(y) .
$$


Conditions (P1) and (P2) are then obvious and (P3) is a condition on the kernel.

The domain of $T$ is defined by $\mathcal{D}(T)=\{f: T|f|<\infty$ a.e. $\}$. It is clear that $T$ extends to a positive linear operator $T: \mathcal{D}(T) \rightarrow L^{0}$.

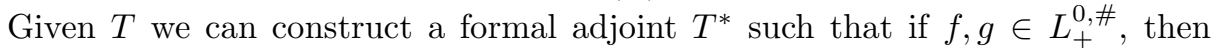
$\int(T f) g d \sigma=\int\left(T^{*} g\right) f d \sigma$. This can be done using the Radon-Nikodym theorem and it is not difficult to check that $T^{*}$ is also a positive operator. To verify (P3) for $T^{*}$ one needs only observe that if $f$ is chosen as in (P3) for $T$ and if $\int u(T f)<\infty$, then $T^{*} u<\infty$ a.e.

We first discuss weighted norm inequalities for $T$. Fix $1<p<\infty$, and let $\frac{1}{p}+\frac{1}{q}=1$.

Let $H$ be the set of $f \in L_{+}^{0}$ such that there exists $0 \leq g \in L^{p}(\sigma)$ with $\|g\|_{p} \leq 1$ and $f \leq(T g)^{p}$.

Lemma 2.4. $H$ is a solid closed convex set. $H$ is bounded if and only if $L^{p} \subset \mathcal{D}(T)$. $H$ is nondegenerate if and only if whenever $f \in L_{+}^{0}$ with $T^{*} f=0$ a.e., then $f=0$ a.e. (i.e. $T^{*}$ is strictly positive).

Proof. Let us prove convexity. If $f_{1}, f_{2} \in H$ and $0 \leq t \leq 1$, we first find $0 \leq g_{j}$ with $T g_{j}^{p} \geq f_{j}$ and $\left\|g_{j}\right\|_{p} \leq 1$ for $j=1,2$. Then let $g=\left(t g_{1}^{p}+(1-t) g_{2}^{p}\right)^{1 / p}$. Then $\|g\|_{p} \leq 1$ and $T g^{p} \geq t f_{1}+(1-t) f_{2}$. $H$ is easily seen to be also solid.

We next check $H$ is closed. Suppose $f_{n} \in H$ and $f_{n} \uparrow f$ a.e. Then there exist $0 \leq g_{n} \in L^{p}$ with $\left\|g_{n}\right\|_{p} \leq 1$ and $\left(T g_{n}\right)^{p} \geq f_{n}$. Since $L^{p}$ is reflexive when $1<p<\infty$, we can find a weak limit point $h$ of $\left(g_{n}\right)$ and a sequence $g_{n}^{\prime} \in$ co $\left\{g_{n}, g_{n+1}, \ldots\right\}$ such that $g_{n}^{\prime} \rightarrow g$ in $L^{p}$-norm. Then $T g_{n}^{\prime} \rightarrow T h$ in $L^{0}$. Now

$$
\left(T g_{n}^{\prime}\right)^{p} \geq \inf \left\{\left(T g_{k}\right)^{p}: k \geq n\right\} \geq f_{n}
$$

so that $(T h)^{p} \geq f$. It follows that $f \in H$ and by Proposition 2.2 the set $H$ is closed.

Clearly if $0 \leq f \in L^{p}$ and $E=\{x: T f(x)=\infty\}$, then $\alpha \chi_{E} \in H$ for every $\alpha>0$. Hence if $H$ is bounded, $L^{p} \subset \mathcal{D}(T)$. Conversely assume $L^{p} \subset \mathcal{D}(T)$. To show $H$ is bounded, let us suppose $2^{n} f_{n} \in H$. The above argument used to show $H$ is closed shows the existence of $0 \leq h \in L^{p}$ with $T h \geq \sum_{n=1}^{\infty} f_{n}$ so that $f_{n} \rightarrow 0$ a.e.

If $H$ is nondegenerate, then there exists $0 \leq f \in L^{p}$ with $T f>0$ a.e. If $T^{*} h=0$ a.e., then $\int h(T f) d \sigma=0$ so that $h=0$ a.e.

Conversely let us show $H$ is nondegenerate, under the hypothesis that $T^{*} f=0$ a.e. implies $f=0$ a.e. Let $\left(f_{n}\right)$ be a sequence dense in the positive quadrant of the unit ball of $L^{p}$. Let $g=\left(\sum_{n=1}^{\infty} 2^{-n} f_{n}^{p}\right)^{1 / p}$. Let $E=\{T g=0\}$. Then $\chi_{E} T f_{n}=0$ a.e. for all $n$ whence $T^{*} \chi_{E}=0$ so that $|E|_{\sigma}=0$.

Definition. The space $\mathcal{V}=\mathcal{V}_{p, T}$ consists of all $f \in L^{0}$ such that there exists $h \in L_{p,+}$ with $|f| \leq(T h)^{p}$ a.e. We define

$$
\|f\|_{\mathcal{V}}=\inf \left\{\int h^{p} d \sigma: 0 \leq h,|f| \leq(T h)^{p}\right\} .
$$

If $L^{p} \subset \mathcal{D}(T)$, then $\mathcal{V}$ is a Banach function space. $\mathcal{V}$ is a nondegenerate Banach function space if in addition $T$ is strictly positive.

Definition. The space $\mathcal{W}=\mathcal{W}_{p, T}$ of $L^{p}$-weights for $T$ is defined to be the space of functions $f \in L^{0}$ such that for some $\gamma$ we have

$$
\int|f||T g|^{p} d \sigma \leq \gamma \int|g|^{p} d \sigma
$$


for all $g \in L^{p}$. We define $\|f\|_{\mathcal{W}}$ to be the least constant $\gamma$ for which the preceding inequality holds.

It is clear that if we have both $L^{p} \subset \mathcal{D}(T)$ and $T$ strictly positive, then $\mathcal{W}$ is the Köthe dual of $\mathcal{V}$, and is thus a Banach function space for the norm $\|f\|_{\mathcal{W}}$. Note that this implies $\mathcal{W}^{\prime}=\mathcal{V}$ as we observed above in Lemma 2.3.

We will also introduce the $q$-convexification of $\mathcal{W}_{p, T}$, say $\mathcal{Y}_{p, T}=\mathcal{W}_{p, T}^{1 / q}$.

Definition. $\mathcal{Y}_{p, T}$ is the space of $w \in L^{0}$ such that $|w|^{q} \in \mathcal{W}_{p, T}$ with the associated norm

$$
\|w\|_{\mathcal{Y}}=\left\||w|^{q}\right\|_{\mathcal{W}}^{1 / q}
$$

This is a Banach function space whenever $\mathcal{W}_{p, T}$ is a Banach function space.

Note that $\|w\|_{\mathcal{Y}}$ is the least constant $\gamma$ for which the inequality

$$
\int w^{q}|T g|^{p} d \sigma \leq \gamma^{q} \int|g|^{p} d \sigma
$$

holds for all $g \in L^{p}$.

We next consider the nonlinear equation

$$
u=T u^{q}+f
$$

where $T$ is, as before, a positive operator and $1<q<\infty$. We suppose $f \geq 0$ and seek a positive solution $u \in L^{0}$. With the restriction that $T$ is a kernel operator but for more general convex functions in place of $u \rightarrow u^{q}$ this problem was previously considered by Baras and Pierre [6].

Let us start with some very elementary observations. We denote by $\mathcal{A}: L_{+}^{0, \#} \rightarrow$ $L_{+}^{0, \#}$ the nonlinear map $\mathcal{A} f=T f^{q}$ and rewrite (2.1) as

$$
u=\mathcal{A} u+f .
$$

Note that $\mathcal{A}$ is a convex operator: $\mathcal{A}(t f+(1-t) g) \leq t \mathcal{A} f+(1-t) \mathcal{A} g$ for all $f, g \geq 0$ and $0<t<1$.

Proposition 2.5. Suppose $f \in L_{+}^{0}$. Define $u_{0}=0$ and then $u_{n}=\mathcal{A} u_{n-1}+f$ for $n \geq 1$. Then the following are equivalent:

(1) There exists $v \in L_{+}^{0}$ with $v=\mathcal{A} v+f$.

(2) The sequence $\left(u_{n}\right)$ is bounded in $L^{0}$.

(3) $\sup u_{n}<\infty$ a.e. $n \geq 0$

(4) There exists $w \in L_{+}^{0}$ with $w \geq \mathcal{A} w+f$.

Proof. Of course (1) implies (4). (4) implies (3) since we will have $0 \leq u_{n} \leq w$ for all $n$. (3) trivially implies (2). If $(2)$ holds, then since $\left(u_{n}\right)$ is increasing we have $v=\sup _{n \geq 0} u_{n} \in L^{0}$ solving $(2.1)$.

Definition. We define $S=S_{q, T}$ to be the set of all $f \geq 0$ so that (2.1) has a solution.

Notice that the iterative procedure of Proposition 2.5 yields a minimal solution $u$ of (2.1) corresponding to each $f \in S$ and that the map $f \rightarrow u$ (where $u$ is the minimal solution) is monotone.

Proposition 2.6. The set $S$ is a solid convex set. 
Proof. This follows from 2.5. The only part requiring proof is convexity. If $f, g \in S$ and $0 \leq t \leq 1$, then there exist $u, v$ with $u=\mathcal{A} u+f, v=\mathcal{A} v+g$. But then using the convexity of $\mathcal{A}$ we have $t u+(1-t) v \geq \mathcal{A}(t u+(1-t) v)+t f+(1-t) g$ so that $t f+(1-t) g \in S$.

Proposition 2.7. (1) Suppose $f \in S$. Then $\sup _{n \geq 0} \mathcal{A}^{n} f<\infty$ a.e.

(2) Suppose co $\left\{\mathcal{A}^{n} f: n \geq 0\right\}$ is bounded in $L^{0}$. Then $p^{-1} q^{1-p} f \in S$. In particular, if $\sup _{n \geq 0} \mathcal{A}^{n} f<\infty<\infty$ a.e. (e.g. if for some $n$ we have $\mathcal{A}^{n} f \leq \mathcal{A}^{n-1} f$ ), then $p^{-1} q^{1-p} f \in S$.

(3) If $\mathcal{A} f \leq q^{-1} p^{1-q} f$, then (2.1) has a solution u such that $f \leq u \leq p f$.

(4) If $\mathcal{A}^{2} f \leq q^{-q} p^{q(1-q)} \mathcal{A} f$, then (2.1) has a solution u such that

$$
f+\mathcal{A} f \leq u \leq f+p^{q} \mathcal{A} f .
$$

Proof. (1) is immediate. If $u=\mathcal{A} u+f$, then $\mathcal{A}^{n} f \leq \mathcal{A}^{n} u \leq u$.

For (2) let $G$ be the set co $\left\{\mathcal{A}^{n} f: n \geq 0\right\}$. Define $u_{0}=0$ and then $u_{n}=$ $\mathcal{A} u_{n-1}+p^{-1} q^{1-p} f$ for $n \geq 1$. We will show by induction that there is a sequence $v_{n} \in G$ such that $u_{n} \leq q^{-p / q} v_{n}$. This is trivial for $n=0$; now assume it is proved for $n=k$. Then $\mathcal{A} u_{k} \leq q^{-p} \mathcal{A} v_{k}$ so that

$$
u_{k+1}=\mathcal{A} u_{k}+p^{-1} q^{1-p} f \in\left((q-1) q^{-p}+q^{-p}\right) G=q^{1-p} G=q^{-p / q} G,
$$

i.e. $u_{n} \leq q^{-p / q} v_{n}$ for all $n$. Hence $\left(u_{n}\right)$ is bounded in $L^{0}$ and we can apply Proposition 2.5.

For (3) define $u_{0}=0$ and $u_{n}=\mathcal{A} u_{n-1}+f$. Then for $n \geq 1$ by induction $f \leq u_{n} \leq c_{n} f$ where $c_{1}=1$ and

$$
c_{n+1}=q^{-1} p^{1-q} c_{n}^{q}+1 .
$$

Since $x_{0}=p$ is the only root of the equation $x=q^{-1} p^{1-q} x^{q}+1$ and $c_{1}=1$, it is easy to see that $\lim _{n \rightarrow \infty} c_{n}=p$ and hence $v=\sup _{n} u_{n}$ is a solution to (2.1) such that $f \leq v \leq p f$.

To prove (4) we will need the inequality

$$
\mathcal{A}(f+g) \leq\left[(\mathcal{A} f)^{1 / q}+(\mathcal{A} g)^{1 / q}\right]^{q}
$$

for all $q \geq 1$ and $f, g \geq 0$. (For the reverse inequality in case $0<q<1$ see [31], p. 55). Note that if $T$ is a kernel operator, then this follows from Minkowski's inequality. For an arbitrary $T$, using the convexity of $\mathcal{A}$ we have

$$
\mathcal{A}(f+g) \leq \lambda \mathcal{A}\left(\frac{f}{\lambda}\right)+\mu \mathcal{A}\left(\frac{g}{\mu}\right)=\lambda^{1-q} \mathcal{A} f+\mu^{1-q} \mathcal{A} g
$$

for all $\lambda, \mu>0, \lambda+\mu=1$. It then follows that we have:

$$
\mathcal{A}(f+g) \leq \inf _{\substack{\lambda+\mu=1 \\ \lambda, \mu>0}}\left\{\lambda^{1-q} \mathcal{A} f+\mu^{1-q} \mathcal{A} f\right\} \quad \text { a.e. }
$$

The desired inequality follows for a.e. $x$ by letting

$$
\lambda=\frac{(\mathcal{A} f(x))^{1 / q}}{(\mathcal{A} f(x))^{1 / q}+(\mathcal{A} g(x))^{1 / q}}, \quad \mu=\frac{(\mathcal{A} g(x))^{1 / q}}{(\mathcal{A} f(x))^{1 / q}+(\mathcal{A} g(x))^{1 / q}},
$$

when $\mathcal{A} f(x), \mathcal{A} g(x)>0$. 
Now for $u_{0}=f$ and $u_{n}=\mathcal{A} u_{n-1}+f$ obviously $u_{n} \geq f+\mathcal{A} f$ if $n \geq 1$. To get the upper estimate we show by induction that $u_{n} \leq f+c_{n} \mathcal{A} f$ where

$$
c_{n}=\left(1+q^{-1} p^{1-q} c_{n-1}\right)^{q} .
$$

This is true for $n=1$ since $c_{1}=0$ and $c_{2}=1$. Assuming it is true for $n=k$, we have

$$
\begin{aligned}
u_{k+1}=\mathcal{A} u_{k}+f & \leq \mathcal{A}\left(f+c_{k} \mathcal{A} f\right)^{q}+f \\
& \leq\left[(\mathcal{A} f)^{1 / q}+c_{k}\left(\mathcal{A}^{2} f\right)^{1 / q}\right]^{q}+f \\
& \leq\left(1+q^{-1} p^{1-q} c_{k}\right)^{q} \mathcal{A} f+f .
\end{aligned}
$$

Hence $u_{k+1} \leq f+c_{k+1} \mathcal{A} f$ where $c_{k+1}=\left(1+q^{-1} p^{1-q} c_{k}\right)^{q}$. It remains to note that $x_{0}=p^{q}$ is obviously the only root of the equation $x=\left(1+q^{-1} p^{1-q} x\right)^{q}$ and $\lim _{n \rightarrow \infty} c_{n}=p^{q}$. Thus $v=\sup _{n} u_{n}$ is a solution to (2.1) with the desired pointwise estimates.

Remark. It is not difficult to see that all the constants in Proposition 2.7 are sharp.

Proposition 2.8. If the set $S$ is bounded, then it is also closed.

Proof. We need only check that if $0 \leq f_{n} \uparrow f$ a.e. and each $f_{n} \in S$, then $f \in S$. Let $u_{n}$ be the minimal solution corresponding to $f_{n}$. Then $\mathcal{A} u_{n} \leq u$ so that, by Proposition 2.6, $p^{-1} q^{1-p} u_{n} \in S$. Thus $\left(u_{n}\right)$ is an increasing sequence which is bounded in $L^{0}$. Let $u=\sup _{n} u_{n}$. Then $u=\mathcal{A} u+f$.

Definition. We define the solution space $\mathcal{Z}_{q, T}$ to be the space of all $f \in L^{0}$ so that for some $\epsilon>0$ we have $\epsilon|f| \in S$. We define

$$
\|f\|_{\mathcal{Z}}=\inf \left\{\alpha>0: \alpha^{-1}|f| \in S\right\} .
$$

We note that $S$ is nondegenerate if there exists $u>0$ a.e. with $K u^{q} \leq u<\infty$ a.e. Let us assume that $S$ is both bounded and nondegenerate. Then $\mathcal{Z}$ is a Banach function space for the norm \|\|$_{Z}$.

Proposition 2.9. Suppose $f \in L_{+}^{0}$. Then $f \in \mathcal{Z}$ if and only if there exists $u \geq f$ and $C>0$ so that $u \in L_{+}^{0}$ and $\mathcal{A} u \leq C u$.

Proof. If $f \in \mathcal{Z}$, then $f /\|f\|_{\mathcal{Z}} \in S$ and so there exists $v \geq f /\|f\|_{\mathcal{Z}}$ with $\mathcal{A} v \leq v$. But then let $u=\|f\|_{\mathcal{Z}} v$. We have $\mathcal{A} u \leq\|f\|_{\mathcal{Z}}^{q-1} u$. Conversely if $\mathcal{A} u \leq C u$, then let $v=C^{-1 /(q-1)}$ so that $\mathcal{A} v \leq v$. Then, by Proposition 2.6, we have $p^{-1} q^{1-p} v \in U$ so that $u \in \mathcal{Z}$.

Remark. If we define

$$
|f|_{\mathcal{Z}}=\inf \left\{\alpha>0: T|f|^{q} \leq \alpha^{q-1}|f|\right\},
$$

then $|f|_{\mathcal{Z}}$ is an equivalent norm on $\mathcal{Z}$.

We next state properties of $\mathcal{Z}$ which will be useful later.

Theorem 2.10. (1) For any $f \in L_{+}^{0}$ we have $f \in \mathcal{Z}$ if and only if $\mathcal{A} f \in \mathcal{Z}$ and

$$
\|f\|_{\mathcal{Z}}^{q} \leq\|\mathcal{A} f\|_{\mathcal{Z}} \leq p q^{p-1}\|f\|_{\mathcal{Z}}^{q}
$$


(2) Suppose $\mathcal{X}$ is a Banach function space which contains $\mathcal{Z}$. Then for any $f \in \mathcal{X}_{+}$we have $f \in \mathcal{Z}$ if and only if $\sup _{n \geq 0}\left\|\mathcal{A}^{n} f\right\|_{\mathcal{X}}^{1 / q^{n}}<\infty$ and

$$
\limsup _{n \rightarrow \infty}\left\|\mathcal{A}^{n} f\right\|_{\mathcal{X}}^{1 / q^{n}} \leq\|f\|_{\mathcal{Z}} \leq p q^{p-1} \limsup _{n \rightarrow \infty}\left\|\mathcal{A}^{n} f\right\|_{\mathcal{X}}^{1 / q^{n}}
$$

(3) If $f \in L_{+}^{0}$, then $f \in Z$ if and only if $\limsup _{n \rightarrow \infty}\left(\mathcal{A}^{n} f\right)^{1 / q^{n}} \in L^{\infty}$ and then

$$
\left\|\limsup _{n \rightarrow \infty}\left(\mathcal{A}^{n} f\right)^{1 / q^{n}}\right\|_{\infty} \leq\|f\|_{\mathcal{Z}} \leq p q^{p-1}\left\|\limsup _{n \rightarrow \infty}\left(\mathcal{A}^{n} f\right)^{1 / q^{n}}\right\|_{\infty}
$$

(4) If $\mathcal{X}$ is as in (2) then,

$$
\limsup _{n \rightarrow \infty}\left\|\mathcal{A}^{n} f\right\|_{\mathcal{X}}^{1 / q^{n}}=\left\|\limsup _{n \rightarrow \infty}\left(\mathcal{A}^{n} f\right)^{1 / q^{n}}\right\|_{\infty}
$$

Proof. (1) Assume first that $f \in \mathcal{Z}$; we may assume $\|f\|_{\mathcal{Z}}=1$. Then there exists $u$ with $u=\mathcal{A} u+f$. Then $u^{q} \geq(\mathcal{A} u)^{q}+f^{q}$ and so $\mathcal{A} u \geq \mathcal{A}^{2} u+\mathcal{A} f$ so that $\|\mathcal{A} f\|_{\mathcal{Z}} \leq 1$. Conversely if $\|\mathcal{A} f\|_{\mathcal{Z}}=1$, then we conclude that there exists $u$ with $u=\mathcal{A} u+\mathcal{A} f$. But then $\mathcal{A}^{n} f \leq u$ for $n \geq 1$, so that by Proposition 2.7, $p^{-1} q^{1-p} f \in S$ or $\|f\|_{\mathcal{Z}} \leq p q^{p-1}$. The result follows by homogeneity.

(2) Note that if $f \in \mathcal{Z}$, then there exists $u \in Z$ with $u \geq f /\|f\|_{\mathcal{Z}}$ with $\mathcal{A} u \leq u$. Hence $\mathcal{A}^{n} f \leq\|f\|_{\mathcal{Z}}^{q^{n}} u$. It follows that

$$
\limsup _{n \rightarrow \infty}\left\|\mathcal{A}^{n} f\right\|_{\mathcal{X}}^{1 / q^{n}} \leq\|f\|_{\mathcal{Z}}
$$

Conversely suppose $\lim \sup _{n \rightarrow \infty}\left\|\mathcal{A}^{n} f\right\|_{\mathcal{X}}^{1 / q^{n}}=a<\infty$. Then if $b>a$, we have that $\left\{\mathcal{A}^{n}\left(b^{-1} f\right)\right\}_{n \geq 0}$ is bounded in $\mathcal{X}$. Applying Proposition 2.7(2) this yields that $p^{-1} q^{1-p} b^{-1} f \in S$ i.e. $f \in \mathcal{Z}$ and $\|f\|_{\mathcal{Z}} \leq b p q^{p-1}$.

(3) Assume $f \in \mathcal{Z}$. Then there exists $u \in \mathcal{Z}_{+}$so that $\mathcal{A}^{n}\left(f /\|f\|_{\mathcal{Z}}\right) \leq u$. Hence

$$
\left(\mathcal{A}^{n} f\right)^{1 / q^{n}} \leq\|f\|_{\mathcal{Z}} u^{1 / q^{n}}
$$

so that

$$
\limsup _{n \rightarrow \infty}\left\|\left(\mathcal{A}^{n} f\right)^{1 / q^{n}}\right\|_{\infty} \leq\|f\|_{\mathcal{Z}}
$$

Conversely if $f \in L_{+}^{0}$ and $\left\|\lim \sup _{n \rightarrow \infty}\left(\mathcal{A}^{n} f\right)^{1 / q^{n}}\right\|_{\infty}=a$, then for any $b>a$ we have $\limsup _{n} \mathcal{A}^{n}\left(b^{-1} f\right)<\infty$ a.e. It follows from Proposition 2.7 that $\|f\|_{\mathcal{Z}} \leq$ $p q^{p-1} b$ and the result follows.

(4) The convexity of the map $\mathcal{A}$ is easily seen to imply that both

$$
\left\|\limsup _{n \rightarrow \infty}\left(\mathcal{A}^{n} f\right)^{1 / q^{n}}\right\|_{\infty} \text { and } \quad \limsup _{n \rightarrow \infty}\left\|\mathcal{A}^{n} f\right\|_{\mathcal{X}}^{1 / q^{n}}
$$

define equivalent norms on $\mathcal{Z}$. Both norms satisfy the identity $\|\mathcal{A} f\|=\|f\|^{q}$. This clearly makes them identical, since one obtains $\left\|\mathcal{A}^{n} f\right\|=\|f\| q^{n}$ and the two norms are equivalent.

Proposition 2.11. $\mathcal{Z}^{\prime}$ is an order-continuous Banach function space so that $\mathcal{Z}^{\prime}$ is separable and $\mathcal{Z}=\mathcal{Z}^{\prime \prime}$ can be identified as the dual space of $\mathcal{Z}^{\prime}$.

Proof. We show that $\mathcal{Z}$ is $q$-convex i.e. there exists a constant $C$ so that if $f_{1}, \ldots, f_{n} \in \mathcal{Z}_{+}$, then $\left\|\left(\sum_{k=1}^{n} f_{k}^{q}\right)^{1 / q}\right\|_{\mathcal{Z}} \leq C\left(\sum_{k=1}^{n}\left\|f_{k}\right\|_{\mathcal{Z}}^{q}\right)^{1 / q}$. Indeed we note as 
in the preceding proposition that $\mathcal{A} f_{k} \leq\left\|f_{k}\right\|_{\mathcal{Z}}^{q-1} f_{k}$. Let $g=\left(\sum_{k=1}^{n} f_{k}^{q}\right)^{1 / q}$. Then

$$
\mathcal{A} g \leq \sum_{k=1}^{n}\left\|f_{k}\right\|_{\mathcal{Z}}^{q-1} f_{k} \leq\left(\sum_{k=1}^{n}\left\|f_{k}\right\|_{\mathcal{Z}}^{q}\right)^{1 / p} g
$$

by Hölder's inequality. Hence again arguing as in the preceding proposition if $h=g /\left(\sum_{k=1}^{n}\left\|f_{k}\right\|_{\mathcal{Z}}^{q}\right)^{1 / q}$, then $\mathcal{A} h \leq h$ so that by Proposition 2.5 , we have $\|h\|_{\mathcal{Z}} \leq$ $p q^{p-1}$. Thus we obtain the desired inequality with constant $C=p q^{p-1}$. (Note that we have in effect proved that the norm $f \rightarrow|f|_{\mathcal{Z}}$ is $q$-convex with constant one.) Now ([31]) this implies that $\mathcal{Z}^{\prime}$ is $p$-concave and in particular order-continuous.

We will now prove a result which is except for certain technical assumptions the same as the main result of Baras and Pierre [6], but our approach is completely different.

Theorem 2.12. If $g \in \mathcal{Z}^{\prime}$, then

$$
\|g\|_{\mathcal{Z}^{\prime}}=p q^{p-1} \inf \left\{\int \frac{h^{p}}{\left(T^{*} h\right)^{p-1}} d \sigma: h \in \mathcal{Z}^{\prime}, h \geq|g|\right\} .
$$

Remark. It follows that if $f \geq 0$ a.e., then $f \in S$ if and only if we have $\int f h d \sigma \leq$ 1 whenever $h \in \mathcal{Z}_{+}^{\prime}$ and $\int\left(h^{p} /\left(T^{*} h\right)^{p-1}\right) d \sigma \leq p^{-1} q^{1-p}$. This is similar to the formulation in [6]. Notice that we do not assume that $T$ is given by a kernel function $K$, which is necessary for the Baras-Pierre approach. Our arguments are purely functional analytic, and depend only on duality. Of course we are restricting our attention to the case of functions of the type $x \rightarrow x^{q}$ while Baras and Pierre consider more general convex functions.

Proof. Let us define $V \subset \mathcal{Z}$ by $V=\left\{f: \exists u \in \mathcal{Z}_{+}: u \geq T u^{q}+f\right\}$. Note that $V \cap \mathcal{Z}_{+}=S$, and that $V$ is convex.

We first show that $V$ is weak*-closed. To do this, by the Banach-Dieudonné theorem it suffices to show that $V \cap \alpha B_{\mathcal{Z}}$ is weak*-closed for all $\alpha>0$. Since $\mathcal{Z}^{\prime}$ is separable, it suffices to consider a sequence $f_{n} \in V \cap \alpha B_{\mathcal{Z}}$ converging to some $f \in \mathcal{Z}$ for the weak*-topology. Thus there exist $u_{n} \in \mathcal{Z}_{+}$with $u_{n}-T u_{n}^{q} \geq f_{n}$. Now since $\alpha^{-1}\left|f_{n}\right| \in S$, there exists $v_{n} \in \mathcal{Z}_{+}$with $v_{n}-T v_{n}^{q}=\alpha^{-1}\left|f_{n}\right|$. Let $(1+\alpha) w_{n}=u_{n}+\alpha v_{n}$. Then by convexity $(1+\alpha) T w_{n}^{q} \leq T u_{n}^{q}+\alpha T v_{n}^{q}$. Hence

$$
(1+\alpha)\left(w_{n}-T w_{n}^{q}\right) \geq f_{n}+\left|f_{n}\right| \geq 0 .
$$

It follows that $\left\|v_{n}\right\|_{\mathcal{Z}},\left\|w_{n}\right\|_{\mathcal{Z}} \leq p q^{p-1}$ and hence $\left\|u_{n}\right\|_{\mathcal{Z}} \leq(1+2 \alpha) p^{-1} q^{1-p}$. The sequence $\left(u_{n}\right)$ is thus also bounded in $\mathcal{Z}$.

By Komlos's theorem we can now pass to a common subsequence, which we still denote by $\left(u_{n}\right)$ and $\left(f_{n}\right)$ so that the sequences $z_{n}=\frac{1}{n}\left(u_{1}+\cdots+u_{n}\right)$ and $g_{n}=\frac{1}{n}\left(f_{1}+\cdots+f_{n}\right)$ are a.e. convergent. If $\varphi \in \mathcal{Z}^{\prime}$ and $\varphi>0$ a.e., then $\left(g_{n}\right)$ is weakly convergent to $f$ in $L^{1}(\varphi)$ and hence it must be also a.e. convergent to $f$.

If $z=\lim _{n} z_{n}$ a.e., then $T z^{q} \leq \liminf _{n} T z_{n}^{q}$ so that $z-T z^{q} \geq \lim \sup _{n} z_{n}-T z_{n}^{q}$. However $z_{n}-T z_{n}^{q} \geq \frac{1}{n} \sum_{k=1}^{n} u_{k}-T u_{k}^{q} \geq g_{n}$. Hence $z-T z^{q} \geq f$ and $f \in V$. This completes the proof that $V$ is weak ${ }^{*}$-closed.

Now let $V^{0}=\left\{h \in \mathcal{Z}^{\prime}: \int h f d \sigma \leq 1 \forall f \in V\right\}$. We show that $h \in V^{0}$ if and only if $h \in \mathcal{Z}_{+}^{\prime}$ and

$$
\int \frac{h^{p}}{\left(T^{*} h\right)^{p-1}} d \sigma \leq p q^{p-1}
$$

First note that it is obvious that $V^{0} \subset \mathcal{Z}_{+}^{\prime}$. 
Suppose $h \in V^{0}$. Select a sequence $u_{n} \in \mathcal{Z}_{+}$so that $u_{n} \uparrow q^{1-p}(h / T h)^{p-1}$ a.e. (Here $0 / 0$ is interpreted as 0.) Then

$$
\int\left(u_{n}-T u_{n}^{q}\right) h d \sigma \leq 1 .
$$

Noting that $u_{n} h \in L^{1}$ we can rewrite this as

$$
\int\left(u_{n} h-u_{n}^{q} T^{*} h\right) d \sigma \leq 1 .
$$

Pointwise we note that $u_{n} h-u_{n}^{q} T^{*} h \uparrow p^{-1} q^{1-p} h^{p} /\left(T^{*} h\right)^{p-1}$. So by the Monotone Convergence Theorem

$$
\int \frac{h^{p}}{\left(T^{*} h\right)^{p-1}} d \sigma \leq p q^{p-1}
$$

Conversely suppose $h \geq 0$ and

$$
\int \frac{h^{p}}{\left(T^{*} h\right)^{p-1}} d \sigma \leq p q^{p-1}
$$

Then if $f \leq u-T u^{q}$ where $u \in \mathcal{Z}_{+}$, we have

$$
\begin{aligned}
\int f h d \sigma & \leq \int\left(u h-h T u^{q}\right) d \sigma \\
& =\int\left(u h-u^{q} T^{*} h\right) d \sigma \\
& \leq \int p^{-1} q^{1-p} \frac{h^{p}}{\left(T^{*} h\right)^{p-1}} d \sigma \leq 1 .
\end{aligned}
$$

Now by the theorem of bipolars (or the Hahn-Banach theorem) we have $V^{00}=V$. We also have $\left(V^{0}-\mathcal{Z}_{+}^{\prime}\right)^{0}=V \cap \mathcal{Z}_{+}=S$. From this we obtain by the Hahn-Banach theorem that $S^{0}$ is the closure in $\mathcal{Z}^{\prime}$ of the convex set $V^{0}-\mathcal{Z}_{+}^{\prime}$. In particular if $g \in S^{\prime}=S^{0} \cap \mathcal{Z}_{+}^{\prime}$ and $\epsilon>0$, then there exist $h \in V^{0}$ and $g^{\prime} \in \mathcal{Z}_{+}^{\prime}$ so that $\left\|g^{\prime}\right\|_{\mathcal{Z}^{\prime}} \leq \epsilon$ and $h+g^{\prime} \geq g$.

Fix $g=g_{0} \in S^{\prime}$ and $\epsilon>0$. Then by induction we can construct sequences $\left(g_{n}\right)$ and $\left(h_{n}\right)$ so that $h_{n} \in \epsilon^{n-1} V^{0},\left\|g_{n}\right\|_{\mathcal{Z}^{\prime}} \leq \epsilon^{n}$ and $g \leq h_{1}+\cdots+h_{n}+g_{n}$. Clearly $g \leq \sum_{n=1}^{\infty} h_{n}=h$ say.

Now

$$
\begin{aligned}
\int \frac{h^{p}}{\left(T^{*} h\right)^{p-1}} d \sigma & \leq\left(\sum_{n=1}^{\infty}\left(\int \frac{h_{n}^{p}}{\left(T^{*} h\right)^{p-1}} d \sigma\right)^{1 / p}\right)^{p} \\
& \leq p q^{p-1}\left(\sum_{n=1}^{\infty} \epsilon^{(n-1) / p}\right)^{p} \\
& \leq p q^{p-1}\left(1-\epsilon^{1 / p}\right)^{-p}
\end{aligned}
$$

Since $\epsilon>0$ is arbitrary, this completes the proof.

\section{QUASI-METRIC KERNELS AND INFINITESIMAL INEQUALITIES}

We will now specialize the positive operator $T$ considered in the previous section. Let $K$ be a positive Borel kernel function $K: X \times X \rightarrow(0, \infty]$ (note that $K(x, y)>0$ 
for all $x, y$ and that $K(x, y)=\infty$ is allowed). We will say that $K$ satisfies the quasimetric inequality if $K$ is symmetric and there is a constant $\kappa \geq 1$ such that for all $x, y$ we have

$$
\frac{1}{K(x, y)} \leq \kappa\left(\frac{1}{K(x, z)}+\frac{1}{K(z, y)}\right)
$$

Under these conditions it is natural to introduce the quasi-metric $\rho(x, y)=$ $(K(x, y))^{-1}$. Note however that we do not assume that $K(x, x)=\infty$ and so $\rho(x, x)>0$ is possible. We can then also introduce the ball of radius $r>0$ i.e.

$$
B_{r}(x)=\{y: \rho(x, y) \leq r\}
$$

but note that this set can be empty.

One large class of examples is created by taking $d$ as a metric on $X$ and $K(x, y)=$ $d(x, y)^{-\alpha}$ for some $\alpha>0$; this kernel defines an operator of fractional integral type. We will refer to a Borel set $B \subset X$ as bounded if $\sup _{x, y \in B} \rho(x, y)<\infty$.

We suppose as in the previous section that there is given a $\sigma$-finite Borel measure $\sigma$ on $X$. Let $\mathcal{M}_{+}(X)$ be the space of all positive $\sigma$-finite Borel measures on $X$. For each $\omega \in \mathcal{M}_{+}(X)$ we can define $K \omega \in L_{+}^{0, \#}(X, \sigma)$ by

$$
K \omega(x)=\int_{X} K(x, y) d \omega(y)
$$

For $f \in L_{+}^{0}(X, \sigma)$ we define

$$
K f(x)=\int_{X} K(x, y) f(y) d \sigma(y)=K \omega(x)
$$

where $d \omega=f d \sigma$. (Thus we identify $L_{+}^{0}$ as a subset of $\mathcal{M}_{+}(X)$.) Sometimes we will write $K f=K^{\sigma} f$ to emphasize that $K$ is defined on $L_{+}^{0}(X, \sigma)$.

It is natural to require that $K$ define a positive operator as described in Section 2 ; this requires only the existence of a strictly positive function $w$ so that $K w<\infty$ a.e.; however, this assumption does not affect the results of the paper except to eliminate triviality.

For $1 \leq s \leq \infty$ we say $f \in L_{\text {loc }}^{s}$ if $f \chi_{B} \in L^{s}$ for every bounded Borel set $B$. We say that $\omega \in \mathcal{M}_{+}(X)$ is locally finite if $|B|_{\omega}<\infty$ for every bounded Borel set $B$.

Our first proposition gives an alternative formula for $K \omega$.

Proposition 3.1. Let $\omega \in \mathcal{M}_{+}(X)$. Then

$$
K \omega(x)=\int_{0}^{\infty} \frac{\left|B_{r}(x)\right|_{\omega}}{r^{2}} d r, \quad x \in X
$$

Proof. For a fixed $x \in X$, we can rewrite $K \omega(x)$, using the distribution function of $K(x, \cdot)$, as

$$
K \omega(x)=\int_{X} K(x, y) d \omega(y)=\int_{0}^{\infty}|\{y: K(x, y)>t\}|_{\omega} d t .
$$

Then the substitution $r=1 / t$ gives

$$
K \omega(x)=\int_{0}^{\infty} \frac{\left|B_{r}(x)\right|_{\omega}}{r^{2}} d r
$$

which proves Proposition 3.1.

Proposition 3.2. If $K$ satisfies the quasi-metric assumption, then $K \omega<\infty$ a.e. implies that $\omega$ is locally finite. 
Proof. If $B$ is a bounded set and $|B|_{\omega}=\infty$, then we can apply the representation 3.2 to deduce that $K \omega(x)=\infty$ everywhere. Indeed for any $x$ there is a large enough $r$ so that $B \subset B_{r}(x)$.

One of our main tools is the following decomposition of $K$ into its "lower" and "upper" parts. For any $a>0$, let

$$
L_{a}(x, y)=\min \left[K(x, y), a^{-1}\right], \quad(x, y) \in X \times X .
$$

If $K$ satisfies the quasi-metric assumption (3.1), then obviously, $L_{a}$ also satisfies (3.1) with the same constant $\kappa$. We now split the kernel into the lower part $L_{a}$ and the upper part $U_{a}=K-L_{a}$.

Proposition 3.3. Suppose $\omega \in \mathcal{M}_{+}(X)$. Then

$$
L_{a} \omega(x)=\int_{a}^{\infty} \frac{\left|B_{r}(x)\right|_{\omega}}{r^{2}} d r, \quad x \in X
$$

and

$$
U_{a} \omega(x)=\int_{0}^{a} \frac{\left|B_{r}(x)\right|_{\omega}}{r^{2}} d r, \quad x \in X .
$$

Proof. By Proposition 3.1, we have

$$
L_{a} \omega(x)=\int_{0}^{\infty} \frac{\left|\widetilde{B_{r}}(x)\right|_{\omega}}{r^{2}} d r,
$$

where $\widetilde{B_{r}}(x)$ is a ball associated with the kernel $L_{a}$. Obviously, $\widetilde{B_{r}}(x)=B_{r}(x)$ if $r>a$, and $\widetilde{B_{r}}(x)=\emptyset$ if $r \leq a$. This yields (3.4), and hence

$$
U_{a} \omega(x)=K \omega(x)-\int_{a}^{\infty} \frac{\left|B_{r}(x)\right|_{\omega}}{r^{2}} d r=\int_{0}^{a} \frac{\left|B_{r}(x)\right|_{\omega}}{r^{2}} d r .
$$

The proof of Proposition 3.2 is complete.

As we will see in the next proposition, the truncated kernel $L_{a}$ or lower part of the operator has certain stability properties for kernels satisfying the quasi-metric inequality: in particular $L_{a} \omega$ obeys a Harnack-type inequality on a ball $B_{a}(x)$ and $\left(L_{a} \omega\right)^{-1}$ is a quasi-concave function of $a>0$.

Proposition 3.4. Suppose that $K$ satisfies the quasi-metric condition (3.1) with a constant $\kappa \geq 1$. Suppose $\omega \in \mathcal{M}_{+}(X)$.

(1) For all $a, b>0$,

$$
L_{a} \omega(x) \leq \max \left(1, \frac{b}{a}\right) L_{b} \omega(x) .
$$

(2) For all balls $B_{a}(x)$,

$$
\frac{1}{2 \kappa} \sup _{B_{a}(x)} L_{a} \omega \leq L_{a} \omega(x) \leq 2 \kappa \inf _{B_{a}(x)} L_{a} \omega .
$$

Remark. It follows from (ii) that if $K$ is an operator in the sense defined in Section 2 so that for some $w>0$ a.e. we have $K w<\infty$ a.e., then we must have that $\sigma$ is locally finite. 
Proof. If $a \geq b$, then obviously $L_{a} \omega \leq L_{b} \omega$. Suppose $a<b$, so that $\delta=b / a>1$. Then the substitution $t=\delta r$ gives

$$
\begin{aligned}
L_{a} \omega(x) & =\int_{a}^{\infty} \frac{\left|B_{r}(x)\right|_{\omega}}{r^{2}} d r=\delta \int_{b}^{\infty} \frac{\left|B_{t / \delta}(x)\right|_{\omega}}{t^{2}} d t \\
& \leq \delta \int_{b}^{\infty} \frac{\left|B_{t}(x)\right|_{\omega}}{t^{2}} d t=\frac{b}{a} L_{a} \omega(x) .
\end{aligned}
$$

This proves statement (1).

To prove statement (2), notice that if $y \in B_{a}(x)$, then by (3.1) $B_{r}(y) \subset B_{2 \kappa r}(x)$ for all $r \geq a$. Hence

$$
\begin{aligned}
& L_{a} \omega(y)=\int_{a}^{\infty} \frac{\left|B_{r}(y)\right|_{\omega}}{r^{2}} d r \leq \int_{a}^{\infty} \frac{\left|B_{2 \kappa r}(x)\right|_{\omega}}{r^{2}} d r \\
= & 2 \kappa \int_{2 \kappa a}^{\infty} \frac{\left|B_{r}(x)\right|_{\omega}}{r^{2}} d r=2 \kappa K_{2 \kappa a} \omega(x) \leq 2 \kappa L_{a} \omega(x) .
\end{aligned}
$$

This proves the lower estimate in (3.7). The upper estimate is proved in a similar manner. The proof of Proposition 3.3 is complete.

Now let us fix $1<q<\infty$ and consider the nonlinear equation

$$
u=K u^{q}+f
$$

for $u \geq 0, f \geq 0$. As in the previous section let $S=S_{q, K}$ be the set of $f$ for which (3.8) has a solution $u \in L_{+}^{0}$.

Proposition 3.5. If $K$ satisfies the quasi-metric assumption, then either $S=\{0\}$ or $S$ is bounded and nondegenerate. Furthermore, if $S \neq\{0\}$, then for any bounded Borel set $B$ there exists $\epsilon>0$ so that $\epsilon \chi_{B} \in S$.

Proof. If $S \neq\{0\}$, then there exists $u \neq 0$ such that $u \geq K u^{q}$. But $K u^{q}>0$ a.e. so that $u>0$ a.e. Now by Proposition 2.7 we have $p q^{p-1} u \in S$ since $\mathcal{A}^{n} u \leq u$ for all $n \in \mathbb{N}$. This shows that $S$ is nondegenerate.

To check boundedness fix $x \in X$ and suppose that for some $a>0$ we have $\left|B_{a}(x)\right|_{\sigma}>0$. Suppose $0 \neq f \in S$ and so $f \leq u$ where $K u^{q} \leq u$. Then $u>0$ a.e., and $u \in L_{l o c}^{q}$. Further

$$
L_{a} u^{q}(x) \geq a^{-1} \int_{B_{a}(x)} u(x)^{q} d \sigma .
$$

By Proposition 3.4 this yields that

$$
u \geq L_{a} u^{q} \geq \frac{1}{2 \kappa a}\left(\int_{B_{a}(x)} u^{q} d \sigma\right) \chi_{B_{a}(x)} .
$$

We deduce immediately that $\left|B_{a}(x)\right|_{\sigma}<\infty$ (so that $\sigma$ must be locally finite if $S \neq\{0\})$. We also note that $\epsilon \chi_{B_{a}(x)} \leq u$ for some $\epsilon>0$ which justifies the last statement in the proposition.

We continue the proof that $S$ is bounded; we have

$$
\int_{B_{a}(x)} u^{q} d \sigma \leq 2 \kappa a\left|B_{a}(x)\right|_{\sigma}^{-1} \int_{B_{a}(x)} u d \sigma .
$$


Applying Hölder's inequality

$$
\int_{B_{a}(x)} u d \sigma \leq\left|B_{a}(x)\right|_{\sigma}^{1 / p}\left(\int_{B_{a}(x)} u^{q} d \sigma\right)^{1 / q} .
$$

Combining we get an estimate:

$$
\int_{B_{a}(x)} u^{q} d \sigma \leq(2 \kappa a)^{p}\left|B_{a}(x)\right|_{\sigma}^{-p / q}
$$

Since this holds for any such ball, it follows that the set $S$ is bounded in $L^{0}$.

Thus $\mathcal{Z}_{q, K}$ either reduces to $\{0\}$ or is a nondegenerate Banach function space with the norm induced by $S_{q, K}$. We also have $\chi_{B} \in \mathcal{Z}$ for any metric ball $B=B_{a}(x)$. We now prove an estimate on $\left\|\chi_{B}\right\|_{\mathcal{Z}}$; this is somewhat more complicated and requires some preliminary work.

Lemma 3.6. Suppose $x \in X$ and $a>0$. Let $B=B_{a}(x)$ and then $B_{j}=B_{a 2^{-j}}(x)$ for $j \in \mathbb{N}$. Let $c_{j}=(4 \kappa a)^{-1} 2^{j}\left|B_{j}\right|_{\sigma}$. Then $K \chi_{B} \geq \phi_{B}$ where $\phi_{B}=\sum_{j \geq 0} c_{j} \chi_{B_{j}}$.

Proof. Let $b=4 \kappa a$. We have

$$
\begin{aligned}
K \chi_{B}(y) & =\int_{0}^{\infty} \frac{\left|B \cap B_{r}(y)\right|_{\sigma}}{r^{2}} d r \\
& \geq \sum_{j=0}^{\infty} \int_{b 2^{-(j+1)}}^{b 2^{-j}} \frac{\left|B \cap B_{r}(y)\right|_{\sigma}}{r^{2}} d r \\
& \geq \frac{1}{b} \sum_{j=0}^{\infty} 2^{j}\left|B \cap B_{b 2^{-(j+1)}}(y)\right|_{\sigma} \\
& \geq \frac{1}{b} \sum_{j=0}^{\infty} 2^{j}\left|B_{j}\right|_{\sigma} \chi_{B_{j}}(y)
\end{aligned}
$$

since if $y \in B_{j}$, then $B_{j} \subset B \cap B_{b 2-(j+1)}$.

Lemma 3.7. Suppose $0<s<\infty$. Then

$$
K \phi_{B}^{s} \geq \frac{1}{(s+1)} \phi_{B}^{s+1} .
$$

Proof. Let $\phi=\phi_{B}$. We also let $\beta_{j}=\sum_{i=0}^{j} c_{i}$ (and $\beta_{-1}=0$ ). We will use the inequality that $\beta_{j}^{s+1}-\beta_{j-1}^{s+1} \leq(s+1) c_{j} \beta_{j}^{s}$.

We start with the observation that

$$
\phi^{s}=\sum_{j=0}^{\infty} \alpha_{j} \chi_{B_{j}}
$$


where $\alpha_{j}=\beta_{j}^{s}-\beta_{j-1}^{s}$. Note that $K \chi_{B_{j}} \geq \sum_{i=j}^{\infty} c_{i} \chi_{B_{i}}$ by Lemma 3.6. It follows that

$$
\begin{aligned}
K \phi^{s} & \geq \sum_{j=0}^{\infty} \alpha_{j} \sum_{i=j}^{\infty} c_{i} \chi_{B_{i}} \\
& =\sum_{i=0}^{\infty} c_{i} \sum_{j=0}^{i} \alpha_{j} \chi_{B_{i}} \\
& =\sum_{i=0}^{\infty} c_{i} \beta_{i}^{s} \chi_{B_{i}} \\
& \geq \frac{1}{s+1} \sum_{i=0}^{\infty}\left(\beta_{i}^{s+1}-\beta_{i-1}^{s+1}\right) \chi_{B_{i}} \\
& =\frac{1}{s+1} \phi^{s+1} .
\end{aligned}
$$

This completes the proof.

Now we introduce the quantity

$$
M(x, a)=\int_{0}^{a} \frac{\left|B_{r}(x)\right|_{\sigma}}{r^{2}} d r=U_{a}(\mathbf{1})(x) .
$$

Here $\mathbf{1}=\chi_{X}$. Thus $U_{a}(\mathbf{1})=U_{a} \sigma$.

Theorem 3.8. If $\mathcal{Z} \neq\{0\}$ and $B=B_{a}(x)$, then

$$
C(q)\left(\frac{M(x, a)}{4 \kappa}\right)^{p / q} \leq\left\|\chi_{B}\right\|_{\mathcal{Z}}
$$

Proof. This is trivial if $|B|_{\sigma}=0$. Otherwise, define $\phi=\phi_{B}$ as in Lemmas 3.6 and 3.7. Let $\mathcal{A} f=K f^{q}$ as in Section 2. Then $\mathcal{A} \chi_{B} \geq \phi$ and $\mathcal{A} \phi^{s} \geq(s q+1)^{-1} \phi^{s q+1}$. It follows by induction that

$$
\begin{aligned}
\mathcal{A}^{n} \chi_{B} & \geq \prod_{j=1}^{n-1}\left(1+q+q^{2}+\cdots+q^{j}\right)^{-q^{n-j-1}} \phi^{1+q+\cdots+q^{n-1}} \\
& =C(n, q) \phi^{\left(q^{n}-1\right) /(q-1)} .
\end{aligned}
$$

Then

$$
\limsup _{n \rightarrow \infty}\left(\mathcal{A}^{n} \chi_{B}\right)^{1 / q^{n}} \geq \lim _{n \rightarrow \infty} C(n, q)^{1 / q^{n}} \phi^{-1 /(q-1)} .
$$

Clearly,

$$
\begin{aligned}
\lim _{n \rightarrow \infty} C(n, q)^{1 / q^{n}} & =\prod_{j=1}^{\infty}\left(1+q+q^{2}+\cdots+q^{j}\right)^{-q^{-j-1}} \\
& =\prod_{j=1}^{\infty} q^{-j q^{-j-1}} \prod_{j=1}^{\infty}\left(1+q^{-1}+q^{-2}+\cdots+q^{-j}\right)^{-q^{-j-1}} \\
& \geq \prod_{j=1}^{\infty} q^{-j q^{-j-1}} \prod_{j=1}^{\infty}\left(1-q^{-1}\right)^{q^{-j-1}} \\
& =q^{-(q-1)^{2}}\left(1-q^{-1}\right)^{1 / q(q-1)}
\end{aligned}
$$


Thus

$$
\limsup _{n \rightarrow \infty}\left(\mathcal{A}^{n} \chi_{B}\right)^{1 / q^{n}} \geq q^{-(q-1)^{2}}\left(1-q^{-1}\right)^{1 / q(q-1)} \phi^{1 /(q-1)} .
$$

Appealing to Theorem 2.10 we obtain

$$
\left\|\chi_{B}\right\|_{\mathcal{Z}} \geq q^{-(q-1)^{2}}\left(1-q^{-1}\right)^{1 / q(q-1)}\|\phi\|_{\infty}^{p / q} .
$$

It remains to notice that by definition of $\phi$

$$
\begin{aligned}
\|\phi\|_{\infty} & =\sum_{j=0}^{\infty} c_{j} \\
& =\frac{1}{4 \kappa a} \sum_{j \geq 0} 2^{j}\left|B_{j}\right|_{\sigma} \\
& \geq \frac{1}{4 \kappa} \int_{0}^{a} \frac{\left|B_{r}(x)\right|_{\sigma}}{r^{2}} d r .
\end{aligned}
$$

This completes the proof.

Corollary 3.9. A necessary condition for $\mathcal{Z} \neq\{0\}$ is that for any a $>0$ we have $M^{*}(x, a)=\sup _{y \in B_{a}(x)} M(y, a) \in L_{l o c}^{\infty}$.

Proof. Note that if $y \in B_{a}(x)$, then $B_{a}(y) \subset B_{2 \kappa a}(x)$ so that

$$
M(y, a)^{p / q} \leq C(q)(4 \kappa)^{p / q}\left\|\chi_{B_{2 \kappa a}(x)}\right\|_{\mathcal{Z}}
$$

The following theorem introduces an inequality we name the infinitesimal inequality; this is a necessary condition on a measure $\omega$ so that $K \omega \in \mathcal{Z}$.

Theorem 3.10 (The infinitesimal inequality). Assume that $K$ satisfies the quasimetric condition. Then there is a constant $C=C(\kappa)$ so that if $\omega \in \mathcal{M}_{+}(X)$ satisfies $K \omega \in \mathcal{Z}$, then, for every $x \in X$,

$$
\sup _{a>0}\left\{\int_{0}^{a} \frac{\left|B_{t}(x)\right|_{\sigma}}{t^{2}} d t\right\}^{p / q}\left\{\int_{a}^{\infty} \frac{\left|B_{t}(x)\right|_{\omega}}{t^{2}} d t\right\} \leq C\|K \omega\|_{\mathcal{Z}}
$$

Remarks. (a) The conclusion is that (3.9) holds everywhere: however, when working with the infinitesimal inequality later it will only be necessary to assume that (3.9) holds $\sigma$-a.e. for each $a>0$.

(b) There is an appealing alternative form of this inequality (in the almost everywhere form), namely

$$
\sup _{a>0}\left\|\left(U_{a} \sigma\right)^{1 / q}\left(L_{a} \omega\right)^{1 / p}\right\|_{\infty} \leq C^{\prime}\|K \omega\|_{\mathcal{Z}}^{1 / p} .
$$

Proof. We note that for all $a>0, x, y \in X$ if $B=B_{a}(x)$, then

$$
\chi_{B}(y) L_{a} \omega(x) \leq 2 \kappa L_{a} \omega(y)
$$

by Proposition 3.4. Hence

$$
L_{a} \omega(x)\left\|\chi_{B}\right\|_{\mathcal{Z}} \leq 2 \kappa\|K \omega\|_{\mathcal{Z}}
$$

and by Theorem 3.8

$$
L_{a} \omega(x) M(x, a)^{p / q} \leq C\|K \omega\|_{\mathcal{Z}}
$$

where $C=C(\kappa)$. This yields (3.9). 
Corollary 3.11. There is a constant $C=C(\kappa)$ so that if $f \in \mathcal{Z}_{+}$, then for every $x \in X$, if $d \omega=f^{q} d \sigma$

$$
\sup _{a>0}\left\{\int_{0}^{a} \frac{\left|B_{t}(x)\right|_{\sigma}}{t^{2}} d t\right\}^{1 / q}\left\{\int_{a}^{\infty} \frac{\left|B_{t}(x)\right|_{\omega}}{t^{2}} d t\right\}^{1 / p} \leq C\|f\|_{\mathcal{Z}}^{q-1} .
$$

Proof. Here $K \omega=K f^{q} \in \mathcal{Z}$ so that by Theorem 3.10

$$
\sup _{a>0}\left\{\int_{0}^{a} \frac{\left|B_{t}(x)\right|_{\sigma}}{t^{2}} d t\right\}^{1 / q}\left\{\int_{a}^{\infty} \frac{\left|B_{t}(x)\right|_{\omega}}{t^{2}} d t\right\}^{1 / p} \leq C\left\|K f^{q}\right\|_{\mathcal{Z}}^{1 / p}
$$

However $\left\|K f^{q}\right\| \leq p q^{p-1}\|f\|_{\mathcal{Z}}^{q}$ (Theorem 2.10) and so the result follows.

\section{WEIGHTED NORM INEQUALITIES AND NONLINEAR INTEGRAL EQUATIONS}

In this section we prove some of the main results of the paper (Theorems 4.8 and 4.9). We develop connections between the solvability of (2.1) and weighted norm inequalities, which clarifies the role of the infinitesimal inequalities and testing conditions of Sawyer type. Simple criteria of Koosis type (see [18]) are given for $\mathcal{W}_{p, K}$ and $\mathcal{Z}_{q, K}$ to be nondegenerate Banach function spaces. Sharp estimates of $\left\|\chi_{B}\right\|_{\mathcal{Z}_{q, K}}$ for a ball $B=B_{r}(x)$ are obtained. We retain the assumption that $K$ satisfies the quasi-metric condition (3.1).

We recall from Section 2 that $f \in L^{0}$ is an $L^{p}$-weight for $K$ if there is a constant $\gamma>0$ such that

$$
\int|f||K g|^{p} d \sigma \leq C \int|g|^{p} d \sigma
$$

for all $g \in L^{p}(\sigma)$. The space of $L^{p}$-weights is denoted $\mathcal{W}_{p, K}$. Since $K(x, y)>0$ for all $x, y$ if $K$ defines a positive operator, it is strictly positive and we have that $\mathcal{W}_{p, K}$ under the norm $f \rightarrow\|f\|_{\mathcal{W}}=\inf \{C:$ (4.1) holds $\}$ is a nondegenerate Banach function space on $(X, \sigma)$ provided the assumption that $L^{p} \subset \mathcal{D}(K)$ holds (and this condition is clearly also necessary). Even without this assumption it is clear that $\left\{f:\|f\|_{\mathcal{W}} \leq 1\right\}$ is a bounded subset of $L^{0}$. We recall also that $\mathcal{Y}=\mathcal{Y}_{p, K}=\left\{f:|f|^{q} \in \mathcal{W}_{p, K}\right\} ;$ if $\mathcal{W}$ is a Banach function space, then so is $\mathcal{Y}$ with the associated norm $f \rightarrow\left\||f|^{q}\right\|_{\mathcal{W}}^{1 / q}$.

We first prove a simple criterion for $\mathcal{W}_{p, K}$ to be a Banach function space on $(X, \sigma)$. To do this we introduce the quantity

$$
N(x, a)=\int L_{a}(x, y)^{q} d \sigma(y)=\int_{a}^{\infty} \frac{\left|B_{t}(x)\right|_{\sigma}}{t^{q+1}} d t .
$$

It is immediate that if $N(x, a)<\infty$ for some $x, a$, then $\sigma$ is locally finite and that $N(y, b)<\infty$ for every $y \in X$, and $b>0$. This follows from applying Proposition 3.4 to the modified kernel $K(x, y)^{q}$.

Proposition 4.1. (1) If $L^{p} \subset \mathcal{D}(K)$, then $\sigma$ is locally finite and for every $a>0$, $N(x, a)<\infty$.

(2) If for every $a>0$ and $x \in X$ we have $N(x, a)<\infty$ and $M^{*}(x, a)<\infty$, then $L^{p} \subset \mathcal{D}(K)$ (and hence there exists $w \in \mathcal{W}_{p, K}$ with $w>0$ a.e.). Furthermore we have the estimate

$$
\left\|\chi_{B_{a}(x)}\right\|_{\mathcal{W}} \leq C\left(\left|B_{a}(x)\right|_{\sigma} N(x, a)^{p / q}+M^{*}(x, 2 \kappa a)^{p}\right)
$$

where $C$ depends only on $\kappa$. 
Proof. (1) Pick a ball $B=B_{a}(x)$ with $|B|_{\sigma}>0$. Then we can apply Proposition 3.4 again to get that

$$
K f \geq(2 \kappa)^{-1} L_{a} f(x) \chi_{B}
$$

If $L^{p} \subset \mathcal{D}(K)$, this implies that $L_{a} f(x)<\infty$ for all $f \in L^{p}$ so that $N(x, a)<\infty$. This contradiction implies $N(x, a)<\infty$ for all $x \in X$ and $a>0$.

(2) It will suffice to prove that for every ball $B=B_{a}(x)$ we have $\chi_{B} \in \mathcal{W}$. Note first that if $y \in B$, then by Proposition 3.4 and Hölder's inequality

$$
L_{a} f(y) \leq 2 \kappa L_{a} f(x) \leq 2 \kappa N(x, a)^{1 / q}\|f\|_{p} .
$$

Hence

$$
\int_{B}\left(L_{a} f\right)^{p} d \sigma \leq(2 \kappa)^{p} N(x, a)^{p / q}|B|_{\sigma} \int f^{p} d \sigma .
$$

Now if $y \in B$, then $U_{a} f(y) \leq M(y, a)\|f\|_{\infty} \leq M^{*}(x, a)\|f\|_{\infty}$. Hence

$$
\left\|\chi_{B} U_{a} f\right\|_{\infty} \leq M^{*}(x, a)\|f\|_{\infty}
$$

Also

$$
\int_{B} U_{a} f(y) d \sigma(y) \leq \int_{B_{2 \kappa a}(x)}\left(\int_{B_{a}(y)} K(y, z) d \sigma(y)\right) f(z) d \sigma(y) \leq M^{*}(x, 2 \kappa a)\|f\|_{1} .
$$

By the Riesz interpolation theorem

$$
\int_{B}\left(U_{a} f\right)^{p} d \sigma \leq M^{*}(x, 2 \kappa a)^{p} \int f^{p} d \sigma .
$$

The proposition now follows by combining the estimates for $L_{a} f$ and $U_{a} f$.

Proposition 4.1(2) gives a situation in which $\mathcal{W}$ is nondegenerate which is sufficient to cover our main interests in this paper. However it is possible to prove more general results which can be regarded as extending previous Koosis-type theorems due to Rubio de Francia [42] and Sawyer [43] for the Riesz potential.

Proposition 4.2. Suppose $K$ satisfies the quasi-metric condition and that $\mu$ is a nontrivial $\sigma$-finite Borel measure on $X$ such that for every $x \in X$ and $a>0$ we have

$$
M_{\mu}^{*}(x, a)=\sup _{y \in B_{a}(x)} \int_{0}^{a} \frac{\left|B_{t}(y)\right|_{\mu}}{t^{2}} d t<\infty .
$$

Let $\sigma$ be a $\mu$-continuous $\sigma$-finite Borel measure on $X$. Then for $(X, \sigma)$ we have $\mathcal{W}_{p, K}$ nondegenerate if and only if

$$
N(x, a)=\int_{a}^{\infty} \frac{\left|B_{t}(x)\right|_{\sigma}}{t^{2}} d t<\infty
$$

for some (and hence all) $x \in X, a>0$.

Proof. The necessity of the condition is proved in Proposition 4.1. For the converse, we remark first that we can replace $\mu$ by its $\sigma$-continuous part and hence assume $d \mu=w d \sigma$ for some strictly positive weight function $w$. We must show that if $0 \leq f \in L^{p}(\sigma)$, then $K f<\infty$ a.e. Indeed in this case let $B=B_{a}(x)$ be any ball. Then

$$
\sup _{y \in B} L_{a} f(y) \leq 2 \kappa L_{a} f(x) \leq 2 \kappa N(x, a)^{1 / q}\|f\|_{p} .
$$


Also

$$
\begin{aligned}
\int_{B} w U_{a} f d \sigma & =\int_{y \in B} \int_{B_{2 \kappa a}(x)} U_{a}(x, y) w(y) d \sigma(y) d \sigma(x) \\
& =\int_{B_{2 \kappa a}(x)} M(y, a) f(y) d \sigma(y) \\
& \leq M^{*}(x, 2 \kappa a)\left|B_{2 \kappa a}\right|_{\sigma}^{1 / q}\|f\|_{p} .
\end{aligned}
$$

This shows that $\chi_{B} K f<\infty \sigma$-a.e.

The following result was proved for the special case when $K$ is the Riesz potential in [42] and [43].

Proposition 4.3. Suppose $K$ satisfies the quasi-metric condition and that $\sigma$ is a $\sigma$-finite Borel measure on $X$ such that for every $x \in X$ and $a>0$ we have $M^{*}(x, a)<\infty$. Let $v \in L_{+}^{0}$. In order that there exist $w \in L_{+}^{0}$ with $w>0$ whenever $v>0$ and

$$
\int(K f)^{p} w d \sigma \leq C \int f^{p} v d \sigma
$$

for all $f \in L^{p}(v d \sigma)$, it is necessary and sufficient that

$$
\int L_{a}(x, y)^{q} v(y)^{1-q} d \sigma(y)<\infty
$$

for some (and hence all) $x \in X$ and $a>0$.

Proof. By replacing $X$ by $X_{0}=\{x: v(x)>0\}$ and restricting $\sigma$ we can suppose $v>0$ everywhere. Let $d \nu=v^{1-q} d \sigma$ and let $K^{\nu} f=K\left(v^{-q / p} f\right)$. Then if we put $f=\varphi v^{-q / p}$, then (4.2) becomes equivalent to

$$
\int\left(K^{\nu} \varphi\right)^{p}(w / v) d \nu \leq C \int f^{p} d \nu
$$

and so the result reduces to Proposition 4.2. The necessary and sufficient condition required is that

$$
\int L_{a}(x, y)^{q} d \nu<\infty
$$

for some $x \in X$ and $a>0$.

We may also study weighted norm inequalities for measures $\omega \in \mathcal{M}_{+}(X)$. We define $\widetilde{\mathcal{W}}_{p, K}$ to be the cone of $\omega$ such that for some constant $C>0$ we have

$$
\int|K g|^{p} d \omega \leq C \int|g|^{p} d \sigma
$$

for all $g \in L^{p}$. Of course if $\omega$ is $\sigma$-continuous, then we can write $d \omega=f d \sigma$ and $f$ satisfies (4.1).

The following characterization of (4.3) is due to Sawyer and Wheeden [43], [44] (see also substantial improvements in [45] and [54]) under the hypothesis that $X$ is a homogeneous space in the sense of Coifman-Weiss [11] equipped with a quasimetric $d(x, y)$ and doubling measure $\mu$ such that $\left|B_{2 r}(x)\right|_{\mu} \leq C\left|B_{r}(x)\right|_{\mu}$ (where $\left.B_{r}(x)=\{y: d(x, y)<r\}\right)$ : Suppose $K(x, y)$ is a kernel satisfying the inequalities

$$
K(x, y) \leq C_{1} K\left(x^{\prime}, y\right) \quad \text { if } \quad d\left(x^{\prime}, y\right) \leq C_{2} d(x, y),
$$


and

$$
K(x, y) \leq C_{1} K\left(x, y^{\prime}\right) \quad \text { if } \quad d\left(x, y^{\prime}\right) \leq C_{2} d(x, y)
$$

for some $C_{1}>1$ and $C_{2}>1$. Suppose that all annuli with respect to $d$ are nonempty. Then (4.3) holds if and only if both of the following testing conditions hold:

$$
\int_{X}\left(K \sigma_{B}\right)^{p} d \omega \leq C|B|_{\sigma}
$$

and

$$
\int_{X}\left(K^{*} \omega_{B}\right)^{q} d \sigma \leq C|B|_{\omega}
$$

for all balls $B$ associated with $d$; here $d \sigma_{B}=\chi_{B} d \sigma, K^{*}$ is a formal adjoint, and as usual $1 / p+1 / q=1,1<p<\infty$. Under certain mild assumptions it can be shown that (4.4) and (4.5) are equivalent to similar inequalities with integration over $B$ in place of $X$ on the left-hand sides. (See [46] and [54].)

Observe that if $K(x, y)=1 / d(x, y)$, then $(K 1)$ and $(K 2)$ hold automatically with $C_{1}=C_{2}=1$ and thus the testing inequalities (4.4), (4.5) characterize $\widetilde{\mathcal{W}}_{p, K}$. As is shown in [53], under some assumptions on $X$ every kernel $K$ which satisfies (K1) and (K2) is controlled by its symmetric "dyadic" analogue $K^{d}$ which satisfies the quasi-metric inequality and is pointwise smaller than $K$; moreover, weighted norm inequalities for integral operators generated by $K$ and $K^{d}$ hold simultaneously so in the setting of homogeneous spaces our quasi-metric assumptions and (K1)-(K2) are in a sense equivalent.

Remarks. (a) We emphasize that these deep two weight results with difficult and rather technical proofs are not used in the present paper. For our purposes it suffices to use simpler pointwise characterizations of weighted norm inequalities discussed in this section. However, the second testing condition (4.5), with $B$ in place of $X$ on the left-hand side, plays an important role in our approach to the solvability problem for the equation $u=K u^{q}+f$.

(b) It is easy to give a nonsymmetric version of our solvability results in the framework outlined above; we do not consider it here to avoid unnecessary complications. For the applications we have in mind it is more convenient to restrict ourselves to the case of possibly nonsymmetric kernels $K$ such that $K(x, y) \asymp$ $s_{1}(x) K_{0}(x, y) s_{2}(x)$ where $K_{0}(x, y)$ is a quasi-metric kernel and $s_{1}, s_{2}$ are arbitrary positive weight functions. These generalizations are obtained in Section 7 together with applications to nonlinear Dirichlet problems.

We start with the following proposition which is basic to our results and explains the significance of the quasi-metric assumption for weighted norm inequalities.

Proposition 4.4. Let $K$ be a kernel which satisfies the quasi-metric assumption. Then for any $g \in L_{0_{+}}$, and $1 \leq s<\infty$,

$$
(K g)^{s} \leq C K\left(g(K g)^{s-1}\right)
$$

where $C=s(2 \kappa)^{s-1}$.

Remarks. (a) Proposition 4.4 shows that integral operators with quasi-metric kernels resemble Hardy's operator $K g(x)=\int_{0}^{x} g(t) d t$ which obviously has the property $(K g)^{s}=s K\left(g(K g)^{s-1}\right)$. 
(b) A different proof valid for kernels satisfying assumptions (K1) and (K2) is given in [53].

Proof. Let $d \nu=g d \sigma$. By Proposition 3.1,

$$
K g(x)=K \nu(x)=\int_{0}^{\infty} \frac{\left|B_{r}(x)\right|_{\nu}}{r^{2}} d r
$$

Clearly, we have

$$
\begin{aligned}
(K g(x))^{s} & =\left(\int_{0}^{\infty} \frac{\left|B_{r}(x)\right|_{\nu}}{r^{2}} d r\right)^{s} \\
& =s \int_{0}^{\infty}\left(\int_{r}^{\infty} \frac{\left|B_{t}(x)\right|_{\nu}}{t^{2}} d t\right)^{s-1} \frac{\left|B_{r}(x)\right|_{\nu}}{r^{2}} d r \\
& =s \int_{0}^{\infty} \int_{B_{r}(x)}\left(\int_{r}^{\infty} \frac{\left|B_{t}(x)\right|_{\nu}}{t^{2}} d t\right)^{s-1} d \nu(y) \frac{d r}{r^{2}} .
\end{aligned}
$$

We estimate the inside integral by Proposition 3.3. For $y \in B_{r}(x)$, we have

$$
\int_{r}^{\infty} \frac{\left|B_{t}(x)\right|_{\nu}}{t^{2}} d t=L^{r} g(x) \leq 2 \kappa L^{r} g(y) \leq 2 \kappa K g(y),
$$

where $L^{r} g$ is the "lower part" of $K g$. Then

$$
\begin{aligned}
(K g(x))^{s} & \leq s(2 \kappa)^{s-1} \int_{0}^{\infty} \frac{\int_{B_{r}(x)}\left(K^{\sigma} g\right)^{s-1} d \nu(y)}{r^{2}} d r \\
& =s(2 \kappa)^{s-1} K\left(g\left(K^{\sigma} g\right)^{s-1}\right)(x),
\end{aligned}
$$

which completes the proof of Proposition 4.4.

Proposition 4.5. Suppose $K$ satisfies the quasi-metric condition and that $\omega \in$ $\mathcal{M}_{+}(X)$. Suppose $K \omega \in Y_{p, K}$ or equivalently $(K \omega)^{q} \in \mathcal{W}_{p, K}$ is an $L^{p}$-weight for K. Then $\omega \in \widetilde{\mathcal{W}}_{p, K}$ (i.e. (4.3) holds).

Proof. Suppose $0 \leq g \in L^{p}(\sigma)$. Then, if $C$ is the constant of Proposition 4.4,

$$
\begin{aligned}
\int(K g)^{p} d \omega & \leq C \int K\left(g(K g)^{p-1}\right) d \omega \\
& =C \int g(K g)^{p-1} K \omega d \sigma \\
& \leq C\left(\int g^{p} d \sigma\right)^{1 / p}\left(\int(K g)^{p}(K \omega)^{q} d \sigma\right)^{1 / q} \\
& \leq C\left\|(K \omega)^{q}\right\|_{\mathcal{W}}^{1 / q}\|g\|_{p}^{p} \\
& =C\|K \omega\|_{\mathcal{Y}}\left\|_{g}\right\|_{p}^{p} .
\end{aligned}
$$

This completes the proof.

Theorem 4.6. Suppose $f \in L_{+}^{0}$ and $f \in \mathcal{Z}_{q, K}$. Then $f \in \mathcal{Y}_{p, K}$ (so that $f^{q}$ is an $L^{p}$-weight for $\left.K\right)$ and there is a constant $C=C(q, \kappa)$ such that $\|f\|_{\mathcal{Y}} \leq C\|f\|_{\mathcal{Z}}$, or equivalently $\left\|f^{q}\right\|_{\mathcal{W}} \leq C\|f\|_{\mathcal{Z}}^{q}$. 
Proof. Suppose first that $K f^{q} \leq f$. If $f$ is not zero, then $f>0$ a.e.; furthermore under this hypothesis, $\sigma$ is locally finite i.e. $|B|_{\sigma}<\infty$ for all bounded sets $B$. Let $0 \leq g \in L^{\infty}$ with $\|g\|_{p}=1$ be supported in a bounded set $B$. Then note that on $B$

$$
K f(x) \geq \alpha \int_{B} f d \sigma
$$

where $\alpha=\inf \{K(x, y): x, y \in B\}$. Hence $f \geq c>0$ on $B$ and so $\chi_{B} f \leq c^{1-q} f^{q}$ and $K\left(\chi_{B} f\right) \leq c^{1-q} f$. Now for any $1 \leq s<\infty$ if $C_{s}=s(2 \kappa)^{s-1}$,

$$
\begin{aligned}
\int(K g)^{s} f^{q} d \sigma & \leq C_{s} \int K\left(g(K g)^{s-1}\right) f^{q} d \sigma \\
& =C_{s} \int \chi_{B} g(K g)^{s-1} K f^{q} d \sigma \\
& \leq C_{s}\|g\|_{\infty} \int(K g)^{s-1} \chi_{B} f d \sigma . \\
& \leq C_{s} c^{1-q}\|g\|_{\infty} \int(K g)^{s-1} f^{q} d \sigma .
\end{aligned}
$$

Since $|B|_{\sigma}<\infty$, this implies by induction that $K g \in L_{s}$ for every integer $s$ and hence all $s$. We now take $s=p$.

$$
\begin{aligned}
\int(K g)^{p} f^{q} d \sigma & \leq C_{p} \int g(K g)^{p-1} K f^{q} d \sigma \\
& \leq C_{p}\left(\int g^{p} d \sigma\right)^{1 / p}\left(\int(K g)^{p} f^{q} d \sigma\right)^{1 / q} .
\end{aligned}
$$

Since the left-hand side is finite and $\|g\|_{p}=1$, we can now cancel and obtain

$$
\int(K g)^{p} f^{q} d \sigma \leq C_{p}^{p}
$$

so that $f^{q} \in \mathcal{W}_{p, K}$ and $\|f\|_{Y} \leq C=C(p, \kappa)$.

Now if $f \in S=S_{q, K}$, there exists $u \geq f$ with $K u^{q} \leq u$ and hence $\|f\|_{\mathcal{Y}} \leq C$. The general case now follows by homogeneity.

Remark. We conclude that if $\mathcal{Z} \neq\{0\}$, then $\mathcal{W} \neq\{0\}$ and $K$ satisfies the condition $L^{p} \subset \mathcal{D}(K)$. It follows that $W$ and $Y$ are then both Banach function spaces.

It is now possible to characterize the solution space $\mathcal{Z}$ by means of weighted norm inequalities, although the result is rather technical:

Theorem 4.7. Suppose $f \in L_{+}^{0}$. Let $f_{0}=f$, and define $\left(f_{n}\right)$ inductively by $f_{n}=$ $K f_{n-1}^{q}$. In order that $f \in \mathcal{Z}$ it is necessary and sufficient that each $f_{n}^{q} \in \mathcal{W}_{p, K}$ and that if $C_{n}$ is the least constant so that

$$
\int(K g)^{p} f_{n}^{q} d \sigma \leq C_{n} \int g^{p} d \sigma
$$

for all $0 \leq g \in L^{p}$, then $\sup _{n \geq 0} C_{n}^{1 / q^{n}}<\infty$.

Proof. It is a direct consequence of Theorem 2.10 that $f \in \mathcal{Z}$ if and only if $\sup _{n}\left\|\mathcal{A}^{n} f\right\|_{Y}^{1 / q^{n}}<\infty$. (This proof tacitly assumes that $\mathcal{Z} \neq\{0\}$; however this case can be argued similarly since the set of $h$ such that $\|h\|_{\mathcal{W}} \leq 1$ is bounded in $\left.L^{0}\right)$. 
Theorem 4.8. Let $\omega \in \mathcal{M}_{+}(X)$. Consider the following conditions on $\omega$ :

(1) $\omega \in \widetilde{\mathcal{W}}_{p, K}$ i.e. there is a constant $C$ so that

$$
\int(K g)^{p} d \omega \leq C \int g^{p} d \sigma
$$

for all $0 \leq g \in L^{p}(\sigma)$.

(2) $\omega$ satisfies the second testing inequality (4.5) i.e. there is a constant $C$ so that for every ball $B$ we have

$$
\int_{B}\left(K \omega_{B}\right)^{q} d \sigma \leq C|B|_{\omega} .
$$

(3) $\omega$ satisfies the infinitesimal inequality (3.9) a.e., i.e. for some constant $C$ and $\sigma$-a.e. $x \in X$,

$$
\sup _{a>0}\left\{\int_{0}^{a} \frac{\left|B_{t}(x)\right|_{\sigma}}{t^{2}} d t\right\}^{p / q}\left\{\int_{a}^{\infty} \frac{\left|B_{t}(x)\right|_{\omega}}{t^{2}} d t\right\} \leq C .
$$

Then the following are equivalent:

(i) $K \omega \in \mathcal{Z}_{q, K}$.

(ii) For some constant $C$ we have $K(K \omega)^{q} \leq C K \omega<\infty$ a.e.

(iii) $\omega$ satisfies (1) and (3).

(iv) $\omega$ satisfies (2) and (3).

Remarks. (a) It follows from Proposition 2.7 (3) that if $K(K \omega)^{q} \leq q^{-1} p^{1-q} K \omega$, then $K \omega \in S_{q, K}$ i.e. the equation $u=K u^{q}+K \omega$ has a solution $u$ such that $K \omega \leq u \leq p K \omega$.

(b) Let $\mathcal{F}$ be an ideal space of measurable functions (e.g. $\mathcal{F}=L^{r}, 0<r \leq \infty$ ). Then by Theorem 4.8 and the preceding remark, there exists a solution $u \in \mathcal{F}$ of the equation $u=K u^{q}+\epsilon f$ for some $\epsilon>0$ if and only if $K \omega \in \mathcal{F} \cap \mathcal{Z}_{q, K}$.

Proof of Theorem 4.8. That (ii) implies (i) is immediate from Proposition 2.7. For (i) implies (iii), we note first that Theorem 4.7 implies that $K \omega \in \mathcal{Y}_{p, K}$ and so Proposition 4.5 gives $\omega \in \mathcal{W}_{p, K}$ i.e. (1) holds. Theorem 3.10 implies (3) holds. It is trivial that (iii) implies (iv). Hence it remains only to prove that (iv) implies (ii).

We let $C$ denote the constant in both (4.8) and (4.9). Note that if $r>0$,

$$
(K \omega)^{q} \leq 2^{q-1}\left(\left(L_{r} \omega\right)^{q}+\left(U_{r} \omega\right)^{q}\right) .
$$

Let $d \nu=(K \omega)^{q} d \sigma$; for each $r>0$ let $d \mu_{r}=\left(U^{r} \omega\right)^{q} d \sigma$ and $d \lambda_{r}=\left(L_{r} \omega\right)^{q} d \sigma$. Suppose $x \in X$. Let $B_{r}=B_{r}(x)$. Since $K(K \omega)^{q}=K \nu$, we have to prove that

$$
K \nu(x)=\int_{0}^{\infty} \frac{\left|B_{r}\right|_{\nu}}{r^{2}} d r \leq C^{\prime} K \omega(x),
$$

where $C^{\prime}$ depends on $C, q$, and $\kappa$.

It is easy to see that $U_{r} \omega=U_{r} \omega_{B_{2 \kappa r}}$ on $B_{r}$. Using this together with (4.8) we have

$$
\left|B_{r}\right|_{\mu_{r}}=\int_{B_{r}}\left(U_{r} \omega\right)^{q} d \sigma=\int_{B_{r}}\left(U_{r} \omega_{B_{2 \kappa r}}\right)^{q} d \sigma \leq C\left|B_{2 \kappa r}\right|_{\omega} .
$$

Hence

$$
\begin{aligned}
\int_{0}^{\infty} \frac{\left|B_{r}\right|_{\mu_{r}}}{r^{2}} d r & \leq C \int_{0}^{\infty} \frac{\left|B_{2 \kappa r}\right|_{\omega}}{r^{2}} d r \\
& =2 C \kappa K \omega(x) .
\end{aligned}
$$


On the other hand

$$
\left|B_{r}\right|_{\lambda_{r}}=\int_{B_{r}}\left(L_{r} \omega\right)^{q} d \sigma \leq(2 \kappa)^{q}\left(L_{r} \omega(x)\right)^{q}\left|B_{r}\right|_{\sigma}
$$

by Proposition 3.4. Thus

$$
\int_{0}^{\infty} \frac{\left|B_{r}\right|_{\lambda_{r}}}{r^{2}} d r \leq(2 \kappa)^{q} \int_{0}^{\infty}\left(\int_{r}^{\infty} \frac{\left|B_{t}\right|_{\omega}}{t^{2}} d t\right)^{q} \frac{\left|B_{r}\right|_{\sigma}}{r^{2}} d r .
$$

Now we use integration by parts to replace the right-hand side $R$ by

$$
R=q(2 \kappa)^{q} \int_{0}^{\infty}\left(\int_{0}^{t} \frac{\left|B_{\tau}\right|_{\sigma}}{\tau^{2}} d \tau\right)\left(\int_{t}^{\infty} \frac{\left|B_{\tau}\right|_{\omega}}{\tau^{2}} d \tau\right)^{q-1} \frac{\left|B_{t}\right|_{\omega}}{t^{2}} d t .
$$

At this point the infinitesimal inequality (4.9) allows to estimate

$$
R \leq C q(2 \kappa)^{q} \int_{0}^{\infty} \frac{\left|B_{t}\right|_{\omega}}{t^{2}} d t=C q(2 \kappa)^{q} K \omega(x)
$$

Combining the estimates (4.10) and (4.11) and the fact that $\nu \leq 2^{q-1}\left(\lambda_{r}+\mu_{r}\right)$ gives that

$$
\int_{0}^{\infty} \frac{\left|B_{r}\right|_{\nu}}{r^{2}} d r \leq C^{\prime} K \omega(x)
$$

for a suitable constant $C^{\prime}=C C^{\prime \prime}(q, \kappa)$. Thus (iv) implies (ii) as claimed.

Theorem 4.9. Suppose $K$ satisfies the quasi-metric condition. Suppose $f \in L_{+}^{0}$. Then the following conditions are equivalent:

(1) $f \in \mathcal{Z}_{q, K}$ i.e. there exists $\epsilon>0$ such that there is a solution of $u=K u^{q}+\epsilon f$.

(2) There exists $C>0$ such that $K\left(K f^{q}\right)^{q} \leq C K f^{q}<\infty$ a.e.

(3) $f \in \mathcal{Y}_{p, K}$ and the measure $\omega$ given by $d \omega=f^{q} d \sigma$ satisfies the infinitesimal inequality (4.9).

(4) The measure $\omega$ defined by $d \omega=f^{q} d \sigma$ satisfies both the testing condition (4.8) and the infinitesimal condition (4.9).

Remarks. (a) By Proposition 2.7 (4) it follows that if $K\left(K f^{q}\right)^{q} \leq q^{-q} p^{q(1-q)} K f^{q}$, then $f \in S_{q, K}$ i.e. the equation $u=K u^{q}+f$ has a solution $u$ such that

$$
f+K f^{q} \leq u \leq f+p^{q} K f^{q} .
$$

(b) Theorem 4.9 and the preceding remark yield the following criterion of the existence of solutions to the equation $u=K u^{q}+\epsilon f$ belonging to some ideal space of measurable functions $\mathcal{F}$ (e.g. $\mathcal{F}=L^{r}, 0<r \leq \infty$ ): There exists a solution $u \in \mathcal{F}$ (for some $\epsilon \geq 0$ ) if and only if $f, K f^{q} \in \mathcal{Z}_{q, K} \cap \mathcal{F}$.

Proof of Theorem 4.9. This is simply a restatement of Theorem 4.8 once one makes the observation that $f \in \mathcal{Z}_{q, K}$ if and only if $K f^{q} \in \mathcal{Z}_{q, K}$ (either Proposition 2.7 or Theorem 2.10 ).

Theorem 4.10. Suppose $K$ satisfies the quasi-metric condition and that $x \in X$ and $a>0$. Then the following conditions are equivalent:

(1) $\mathcal{Z}_{q, K} \neq\{0\}$.

(2) We have $N(x, a)<\infty$ and $\sup _{r \geq a} r^{-q / p} M^{*}(x, r)<\infty$. 
Proof. Assume (1). Then $\mathcal{W} \supset \mathcal{Z}$ contains a strictly positive function and hence by Proposition 4.1, we have $N(x, a)<\infty$. To prove the second condition we can replace $a$ by any $b \geq a$ where $\left|B_{b}(x)\right|_{\sigma}>0$. Let us therefore suppose $\left|B_{a}(x)\right|_{\sigma}>0$. Then $\chi_{B_{a}(x)} \in \mathcal{Z}$. It follows that we have the infinitesimal inequality for $\chi_{B_{a}(x)}$. Thus

$$
\sup _{y \in X} \sup _{r>0} M(y, r)^{p / q} \int_{r}^{\infty} \frac{\left|B_{a}(x) \cap B_{t}(y)\right|_{\sigma}}{t^{2}} d t \leq C .
$$

Assume $r>a$ and $y \in B_{r}(x)$. Then for $t \geq 2 \kappa r$ we have $B_{t}(y) \supset B_{a}(x)$. Hence

$$
M(y, r)^{p / q}\left|B_{a}(x)\right|_{\sigma} \leq 2 C \kappa r .
$$

This implies the second part of (2).

Conversely assume (2). Then again we can assume that $\left|B_{a}(x)\right|_{\sigma}>0$. By Proposition 4.1 , we have that $\chi_{B_{a}(x)} \in \mathcal{W}$. Now we verify the infinitesimal inequality

$$
\sup _{r>0, y \in X} M(y, r)^{p / q} \int_{r}^{\infty} \frac{\left|B_{a}(x) \cap B_{t}(y)\right|_{\sigma}}{t^{2}} d t<\infty .
$$

Let $C=\sup _{r \geq a} r^{-q / p} M^{*}(x, a)$. Suppose first $y \in B_{2 \kappa a}(x)$. Then if $r \leq a$,

$$
\begin{aligned}
\int_{r}^{\infty} \frac{\left|B_{a}(x) \cap B_{t}(y)\right|_{\sigma}}{t^{2}} d t & \leq \int_{0}^{a} \frac{\left|B_{t}(y)\right|_{\sigma}}{t^{2}} d t+\int_{a}^{\infty} \frac{\left|B_{a}(x)\right|_{\sigma}}{t^{2}} d t \\
& \leq M(y, a)+\frac{\left|B_{a}(x)\right|_{\sigma}}{a} \leq C(\kappa) M^{*}(x, 2 \kappa a) .
\end{aligned}
$$

Hence

$$
M(y, r)^{p / q} \int_{r}^{\infty} \frac{\left|B_{a}(x) \cap B_{t}(y)\right|_{\sigma}}{t^{2}} d t \leq C(\kappa, p) M^{*}(x, 2 \kappa a)^{p} .
$$

If $r>a$,

$$
M(y, r)^{p / q} \int_{r}^{\infty} \frac{\left|B_{a}(x) \cap B_{t}(y)\right|_{\sigma}}{t^{2}} d t \leq M(y, r)^{p / q}\left|B_{a}(x)\right|_{\sigma} r^{-1} \leq C^{p / q}\left|B_{a}(x)\right|_{\sigma} .
$$

Now if $y \notin B_{2 \kappa a}(x)$, we set $b=\rho(x, y) /(2 \kappa)$. It follows from the quasi-metric inequality that $B_{a}(x) \cap B_{t}(y)=\emptyset$ if $t<b$. Hence

$$
\int_{r}^{\infty} \frac{\left|B_{a}(x) \cap B_{t}(y)\right|_{\sigma}}{t^{2}} d t \leq \int_{b}^{\infty} \frac{\left|B_{a}(x)\right|_{\sigma}}{t^{2}} d t=b^{-1}\left|B_{a}(x)\right|_{\sigma} .
$$

Since $y \in B_{4 \kappa b}(x)$, we have

$$
\begin{aligned}
M(y, r)^{p / q} \int_{r}^{\infty} \frac{\left|B_{a}(x) \cap B_{t}(y)\right|_{\sigma}}{t^{2}} d t & \leq b^{-1} M^{*}(x, 4 \kappa b)^{p / q}\left|B_{a}(x)\right|_{\sigma} \\
& \leq 4 \kappa C^{p / q}\left|B_{a}(x)\right|_{\sigma} .
\end{aligned}
$$

Thus the infinitesimal inequality holds and so $\chi_{B_{a}(x)} \in \mathcal{Z}$. But this means that $\mathcal{Z}$ contains a strictly positive function.

There is an implicit two-sided estimate of $\left\|\chi_{B_{a}(x)}\right\|_{\mathcal{Z}}$ in the preceding theorem:

Corollary 4.11. Suppose $K$ satisfies the quasi-metric condition and that $x \in X$ and $a>0$. Let

$$
\begin{gathered}
\Phi(x, a)=\left|B_{a}(x)\right|_{\sigma}^{1 / q} N(x, a)^{p / q^{2}}+M^{*}(x, a)^{p / q} \\
+\left|B_{a}(x)\right|_{\sigma}^{1 / q} \sup _{r \geq a} r^{-1 / q} M^{*}(x, r)^{p / q^{2}} .
\end{gathered}
$$


Then

$$
C_{1} \Phi\left(x, \frac{a}{2 \kappa}\right) \leq\left\|\chi_{B_{a}(x)}\right\|_{\mathcal{Z}} \leq C_{2} \Phi(x, 2 \kappa a),
$$

where $C_{1}$ and $C_{2}$ depend only on $\kappa$ and $p$.

Remarks. (a) It is not difficult to see that if $\sigma$ is doubling, then

$$
\begin{gathered}
\left\|\chi_{B_{a}(x)}\right\|_{\mathcal{Z}} \asymp\left|B_{a}(x)\right|_{\sigma}^{1 / q} N(x, a)^{p / q^{2}}+M^{*}(x, a)^{p / q} \\
+\left|B_{a}(x)\right|_{\sigma}^{1 / q} \sup _{r \geq a} r^{-1 / q} M^{*}(x, r)^{p / q^{2}},
\end{gathered}
$$

where the constants of equivalence depend only on $\kappa$ and $p$.

(b) It follows from Corollary 4.11 that $\|\mathbf{1}\|_{\mathcal{Z}} \asymp\|K \mathbf{1}\|_{L^{\infty}}^{p / q}$.

\section{Capacitary inequalities And CRiteria of Solvability}

In the previous section we saw that if $f \geq 0$ is such that there is a positive solution of the equation

$$
u=K u^{q}+f,
$$

then (if $K$ is a quasi-metric kernel) there is a corresponding weighted norm inequality

$$
\int(K g)^{p} f^{q} d \sigma \leq C \int g^{p} d \sigma
$$

for all $g \geq 0$. This can be rewritten in terms of function spaces as $\mathcal{Z}_{q, K} \subset \mathcal{Y}_{p, K}$.

It is natural to ask for a converse result, i.e. for conditions on $K$ so that the weighted norm inequality (5.2) implies that there exists $\epsilon>0$ such that the equation $u=K u^{q}+\epsilon f$ has a positive solution. This would imply that $\mathcal{Y}_{p, K}=\mathcal{Z}_{q, K}$. The aim of this section is to establish such conditions.

We will use the notion of capacity associated with $(K, p, \sigma)$. We define

$$
\text { Cap } E=\operatorname{Cap}_{p, K}(E)=\inf \left\{\int g^{p} d \sigma: K g \geq \chi_{E}, g \geq 0\right\}
$$

where we stress that we require $K g(x) \geq 1$ for every (not almost every) $x \in E$. The theory of these capacities and the corresponding potential theory (which is usually called nonlinear potential theory in the nonclassical case $p \neq 2$ ) is due to B. Fuglede, N. G. Meyers, V. G. Maz'ya and V. P. Havin, Yu. G. Reshetnyak; a weighted theory, for $d \sigma=v d x$, where $v \in A_{\infty}$, was developed by D. Adams [1] (see also [2], [24], [35], [57], and the literature cited there).

If $\omega \in \mathcal{M}_{+}(X)$, then the capacity condition

$$
|E|_{\omega} \leq C \text { Cap } E
$$

for every Borel set $E$ is easily seen to be equivalent to the weak-type weighted norm inequality,

$$
|\{K g(x) \geq \lambda\}|_{\omega} \leq C \lambda^{-p}\left(\int g^{p} d \sigma\right)^{1 / p}
$$

for all $g \geq 0$. In general (5.3) does not imply the corresponding strong-type inequality

$$
\int(K g)^{p} f^{q} d \omega \leq C \int g^{p} d \sigma
$$


but in certain cases this is true. In particular (5.3) implies (5.5) for Riesz potentials or more general convolution operators with radial decreasing kernels on $\mathbf{R}^{n}$ if $\sigma \in$ $A_{\infty}$; see [2], [21] and [34]. In this section we will find some new classes of measures $\sigma$ for which this implication holds (see also [52]).

We will also introduce another concept of capacity given by

$$
\text { cap } E=\inf \left\{\int g^{p} d \sigma: K g \geq \chi_{E}, \sigma \text {-a.e., } g \geq 0\right\} \text {. }
$$

It is clear cap $E \leq$ Cap $E$; in fact cap $E=\inf \left\{\operatorname{Cap} F: F \subset E,|E \backslash F|_{\sigma}=0\right\}$. We also note that cap $E=\left\|\chi_{E}\right\|_{W^{\prime}}$ by applying the results of Section 2. If $\omega$ is absolutely continuous with respect to $\sigma$, then (5.3) is equivalent to

$$
|E|_{\omega} \leq C \operatorname{cap} E
$$

for every Borel set $B$.

We first observe that the capacity condition implies the second testing inequality.

Proposition 5.1. If $\omega$ satisfies the capacity condition (5.3), then $\omega$ satisfies the testing condition (4.8).

Proof. By the remarks above, and duality $([31]) K: L^{q, 1}(\omega) \rightarrow L^{q}(\omega)$ is bounded where $L^{q, 1}$ is the Lorentz space of all Borel functions $f$ such that

$$
\int_{0}^{\infty} t^{1 / q-1} f^{*}(t) d t<\infty
$$

(here $f^{*}$ is the decreasing rearrangement of $f$ ). One immediately obtains (4.5) by applying $K$ to a characteristic function.

Proposition 5.2. Suppose $E$ is a Borel subset of $X$. Assume that $L^{p} \subset \mathcal{D}(K)$ so that $\mathcal{W}_{p, K}$ is a Banach function space on $X$. Then given any Borel set $E$ with cap $E<\infty$ and any $\epsilon>0$ there exists $w \in L^{1}(E, \sigma)$ with $\int_{E} w d \sigma=$ cap $E$ and so that we have

$$
\int(K f)^{p} w d \sigma \leq(1+\epsilon) \int f^{p} d \sigma
$$

for all $f \in L^{p}(\sigma)$.

Proof. This follows from our earlier identification of $\mathcal{W}^{\prime}=\mathcal{V}$ (see Section 2). From the definition of cap $E$ we have $\left\|\chi_{E}\right\|_{\mathcal{V}}=$ cap $E$ and so there exists $w \in \mathcal{W}$ with $\|w\|_{\mathcal{W}} \leq 1+\epsilon$ and $\int w \chi_{E}=\operatorname{cap} E$. Replacing $w$ by $w \chi_{E}$ gives the result.

Our next result is closely related to the concept of an equilibrium measure (see, for example, [2] in the case of Riesz potentials and $d \sigma=d x)$.

Proposition 5.3. Let us assume that $K$ is a quasi-metric kernel such that $\rho=$ $K^{-1}$ is continuous. Suppose also that $\sigma$ is locally finite. Then for any compact set $E$ with Cap $E<\infty$ there is a Borel measure $\omega$ supported on $E$ such that $|E|_{\omega}=$ Cap $E$ and we have the inequality

$$
\int(K f)^{p} d \omega \leq \int f^{p} d \sigma
$$

for every $f \in L^{p}(\sigma)$. 
Proof. Without loss of generality we may assume Cap $E>0$. Fix any $x_{0} \in X$. For all $r>0$ and $s>0$ it is easily seen that if $f \in L^{p}\left(B_{r}\left(x_{0}\right)\right)$, then $L_{s} f$ is continuous on $E$. Since $L_{s}(x, y)$ is continuous and bounded, this follows immediately from the Dominated Convergence Theorem.

Now consider the convex set $F$ of all functions in the space of continuous realvalued functions on $E, \mathcal{C}(E)$ of the form $\sum_{i=1}^{n}\left(L_{s_{i}} f_{i}\right)^{p}-\chi_{E}$ where $0<s_{i}<a$, $f_{i} \in B_{r_{i}}\left(x_{0}\right)$ for some $a<r_{i}<\infty$ and $\sum \int f_{i}^{p} d \sigma \leq$ Cap $E$. Let $P=\{f \in \mathcal{C}(E)$ : $f(x)>0 \forall x \in E\}$. We claim that $F \cap P=\emptyset$. Indeed if not there exist $s_{i}, f_{i}$ as above and $\epsilon>0$ so that

$$
\sum_{i=1}^{n}\left(L_{s_{i}} f_{i}\right)^{p} \geq(1+\epsilon) \chi_{E}
$$

But then

$$
K\left(\sum_{i=1}^{n} f_{i}^{p}\right)^{1 / p} \geq\left(\sum_{i=1}^{n}\left(K f_{i}\right)^{p}\right)^{1 / p} \geq(1+\epsilon)^{1 / p} \chi_{E}
$$

which contradicts the definition of capacity.

Now since the cone $P$ is open, the Hahn-Banach theorem and Riesz representation theorem combine to give a measure $\omega$ such that $\int h d \omega>0$ for all $f \in P$ and $\int h d \omega \leq 0$ for all $f \in F$. If we normalize so that $|E|_{\omega}=\operatorname{Cap} E$, then we have

$$
\int\left(L_{s} f\right)^{p} d \omega \leq 1
$$

whenever $s>0, f$ has bounded support and $\int f^{p} d \sigma \leq 1$. This implies the result.

Now let us introduce for $x \in X$ and $a>0$ the quantity

$$
N(x, a)=\int_{a}^{\infty} \frac{\left|B_{t}(x)\right|_{\sigma}}{t^{1+q}} d t=\int_{X} L_{a}(x, y)^{q} d \sigma(y) .
$$

Definition. We shall say that a locally finite Borel measure $\mu$ is stable with respect to $K$ if there exist constants $C>0$ and $\delta>0$ so that for every $x \in X$ and $0<r<R<\infty$ we have

$$
\left|B_{2 R}(x)\right|_{\mu} \leq C\left|B_{R}(x)\right|_{\mu} \quad \text { and } \quad\left|B_{r}(x)\right|_{\mu} \leq C\left(\frac{r}{R}\right)^{1+\delta}\left|B_{R}(x)\right|_{\mu} .
$$

We remark that of course if $K$ is a Riesz potential on $\mathbf{R}^{n}$ (i.e. $K(x, y)=$ $c\|x-y\|^{-\alpha}$ where $\left.0<\alpha<n\right)$, then Lebesgue measure $\lambda$ is stable for $K$. More generally if $X$ is a space of homogeneous type with quasi-metric $d$ and a doubling measure $\mu$, then $\mu$ is stable for $K(x, y)=d(x, y)^{-\alpha}$ where $0<\alpha<1$ ([20]).

Theorem 5.4. Suppose $K$ satisfies the quasi-metric condition.

(1) There is a constant $C$ so that for any for any ball $B_{a}(x)$ we have

$$
\text { Cap } B_{a}(x) \leq C N(x, a)^{-p / q} \text {. }
$$

(2) Suppose that there exists a measure $\mu \in \mathcal{M}_{+}(X)$ which is stable for $K$. Then there exists a constant $C$ so that for every ball $B=B_{a}(x)$ we have

$$
C^{-1} N(x, a)^{-p / q} \leq \operatorname{Cap} B_{a}(x) \leq C N(x, a)^{-p / q} .
$$

(3) Suppose that there exists a $\sigma$-continuous measure $\mu$ which is stable for $K$. Then there exists a constant $C$ so that for every ball $B=B_{a}(x)$ we have

$$
C^{-1} N(x, a)^{-p / q} \leq \operatorname{cap} B_{a}(x) \leq \text { Cap } B_{a}(x) \leq C N(x, a)^{-p / q} .
$$


Remarks. In particular we obtain equivalence Cap $B_{a}(x) \sim N(x, a)^{-p / q}$ for the case $\mathbf{R}^{n}$ and any measure $\sigma$; we obtain the further equivalence cap $B_{a}(x) \sim$ $N(x, a)^{-p / q}$ for any $\sigma$ of the form $d \sigma=w(x) d x$ where $w(x)>0$ a.e. For measures in class $A_{\infty}$ this result was previously shown by Adams [1]; the upper estimate (5.6) was proved for arbitrary measures by Turesson [51] in the case of Riesz potentials. For the case $d \sigma=d x$ and more general radial convolution operators, a similar result was obtained by Aikawa (see [4]).

Proof. (1) Let $g(y)=L_{a}(x, y)^{q-1}$. Then

$$
\int g(y)^{p} d \sigma(y)=\int L_{a}(x, y)^{q} d \sigma(y)=N(x, a) .
$$

We also have

$$
K g(x) \geq L_{a} g(x)=\int L_{a}(x, y)^{q} d \sigma(y)=N(x, a) .
$$

Applying Proposition 3.4 we obtain

$$
K g \leq(2 \kappa)^{-1} N(x, a) \chi_{B_{a}(x)} .
$$

Hence Cap $B_{a}(x) \leq(2 \kappa)^{p} N(x, a)^{-p / q}$.

(2) Suppose $g \in L^{p}(\sigma)$ and $K g \geq \chi_{B}$ where $B=B_{a}(x)$. Then

$$
L_{a} g(x) \leq\left(\int L_{a}(x, y)^{q} d \sigma(y)\right)^{1 / q}\left(\int g(y)^{p} d \sigma(y)\right)^{1 / p} .
$$

Then

$$
L_{a} g(x) \leq N(x, a)^{1 / q}\|g\|_{p}
$$

and thus if $y \in B_{a}(x)$,

$$
L_{a} g(y) \leq 2 \kappa N(x, a)^{1 / q}\|g\|_{p}
$$

Hence

$$
\int_{B} L_{a} g d \mu \leq 2 \kappa|B|_{\mu} N(x, a)^{1 / q}\|g\|_{p} .
$$

On the other hand

$$
\begin{aligned}
\int_{B} U_{a} g d \mu & =\int U_{a} \mu_{B} g d \sigma \\
& \leq \int_{B^{*}} U_{a} \mu g d \sigma \\
& \leq\left(\int_{B^{*}}\left(U_{a} \mu\right)^{q} d \sigma\right)^{1 / q}\|g\|_{p},
\end{aligned}
$$

where $B^{*}=B_{2 \kappa a}(x)$.

Now we estimate

$$
U_{a} \mu(y)=\int_{0}^{a} \frac{\left|B_{t}(y)\right|_{\mu}}{t^{2}} d t \leq C_{1}\left|B_{a}(y)\right|_{\mu}
$$

for a suitable constant $C_{1}$ since $\mu$ is stable for $K$. But if $y \in B^{*}$, then $B_{a}(y) \subset$ $B_{\kappa(2 \kappa+1) a}(x)$ so that $\left|B_{a}(y)\right|_{\mu} \leq C_{2}|B|_{\mu}$ again by the stability condition. Hence

$$
\int_{B} U_{a} g d \mu \leq C_{3}|B|_{\mu}\left|B^{*}\right|_{\sigma}^{1 / q}\|g\|_{p}
$$


Observe that $\left|B^{*}\right|_{\sigma}^{1 / q} \leq C_{4} N(x, a)^{1 / q}$ where $C_{4}=C_{4}(\kappa)$. Combining with (5.9) we obtain

$$
\int_{B} K g d \mu \leq C_{5}|B|_{\mu} N(x, a)^{1 / q}\|g\|_{p}
$$

which gives the lower estimate since $K g \geq \chi_{B}$ (everywhere).

(3) The proof is the same as (2) except for the final observation note that if $K g \geq \chi_{B} \sigma$-a.e., then $K g \geq \chi_{B} \mu$-a.e.

Before moving to our main result of this section, let us prove a preliminary lemma.

Lemma 5.5. Suppose $K$ is a quasi-metric kernel with the property that there is a constant $C$ so that for every $x \in X$ and $a>0$ we have $M(x, a) \leq C a^{q-1} N(x, a)$. Then there exists a constant $C^{\prime}$ so that for every $x \in X$ and $a>0$ we have

$$
M(x, a)^{p / q} \int_{a}^{\infty} \frac{N(x, t)^{-p / q}}{t^{2}} d t \leq C^{\prime}
$$

Proof. First we observe if $M(x, a) \leq C a^{q-1} N(x, a)$, then

$$
\int_{0}^{a} \frac{\left|B_{t}(x)\right|}{t^{2}} d t \leq C a^{q-1} \int_{a}^{\infty} \frac{\left|B_{t}(x)\right|}{t^{1+q}} d t \leq C \int_{a}^{\infty} \frac{\left|B_{t}(x)\right|_{\sigma}}{t^{2}} d t
$$

so that for every $x \in X$ we have $\lim _{a \rightarrow \infty} M(x, a)=\infty$. For fixed $x \in X$ and $a>0$ let us define $a^{*}>a$ so that $M\left(x, a^{*}\right)=(1+1 / 2 C) M(x, a)$.

We claim now that $M(x, a) \leq 2 C a^{q-1} N\left(x, a^{*}\right)$. To see this note that

$$
\begin{aligned}
M(x, a) & \leq C a^{q-1} N(x, a) \\
& =C a^{q-1} \int_{a}^{a^{*}} \frac{\left|B_{t}(x)\right|_{\sigma}}{t^{1+q}} d t+C a^{q-1} N\left(x, a^{*}\right) \\
& \leq C\left(M\left(x, a^{*}\right)-M(x, a)\right)+C a^{q-1} N(x, a) \\
& =\frac{1}{2} M(x, a)+C a^{q-1} N(x, a) .
\end{aligned}
$$

The estimate then follows.

Now for fixed $a$ define a sequence $\left(a_{j}\right)_{j=0}^{\infty}$ inductively by $a_{0}=a$ and then $a_{j}=$ $a_{j-1}^{*}$. We have

$$
\begin{aligned}
M(x, a)^{p / q} \int_{a}^{\infty} \frac{N(x, t)^{-p / q}}{t^{2}} d t & =M(x, a)^{p / q} \sum_{j=0}^{\infty} \int_{a_{j}}^{a_{j+1}} \frac{N(x, t)^{-p / q}}{t^{2}} d t \\
& \leq M(x, a)^{p / q} \sum_{j=0}^{\infty} N\left(x, a_{j+1}\right)^{-p / q} a_{j}^{-1} \\
& \leq(2 C)^{p / q} M(x, a)^{p / q} \sum_{j=0}^{\infty} M\left(x, a_{j}\right)^{-p / q} \\
& =(2 C)^{p / q} \sum_{j=0}^{\infty}\left(1+\frac{1}{2 C}\right)^{-p j / q} \\
& =C^{\prime},
\end{aligned}
$$

say. This completes the proof of the lemma. 
We can now state our main theorem of the section:

Theorem 5.6. Let $K$ be a quasi-metric kernel. Assume that $N(x, a)<\infty$ for all $x \in X$ and $a>0$ and that there is a constant $C$ so that for every $x \in X$ and $a>0$,

$$
\int_{0}^{a} \frac{\left|B_{t}(x)\right|_{\sigma}}{t^{2}} d t \leq C a^{q-1} \int_{a}^{\infty} \frac{\left|B_{t}(x)\right|_{\sigma}}{t^{1+q}} d t
$$

(i.e. $\left.M(x, a) \leq C a^{q-1} N(x, a)\right)$. Suppose $\omega \in \mathcal{M}_{+}(X)$. Then $\mathcal{Z}_{q, K} \neq\{0\}$ and the following statements are equivalent:

(1) $\omega \in \widetilde{\mathcal{W}}_{p, K}$ i.e. $\omega$ satisfies the weighted norm inequality

$$
\int|K g|^{p} d \omega \leq C \int|g|^{p} d \sigma
$$

for all $g \in L^{p}(\sigma)$.

(2) $\omega$ satisfies the capacity condition $|E|_{\omega} \leq$ Cap $E$ for all Borel sets B. (Equivalently the weak-type inequality (5.4) holds.)

(3) $\omega$ satisfies the testing condition

$$
\int\left(K \omega_{B}\right)^{q} d \sigma \leq C|B|_{\omega}
$$

for all balls $B$.

(4) $K \omega \in \mathcal{Z}_{q, K}$ i.e. for some $\epsilon>0$ there is a solution $u$ of the equation $u=$ $K u^{q}+\epsilon K \omega$.

(5) There is a constant $C$ so that $K(K \omega)^{q} \leq C K \omega$.

Proof. Let us first prove the nondegeneracy of $\mathcal{Z}_{q, K}$. Fix any $x \in X$ and $a>0$. If $r>a$ and $y \in B_{r}(x)$, then $M(y, r) \leq C r^{q-1} N(y, r) \leq C^{\prime} r^{q-1} N(x, r)$ where $C, C^{\prime}$ do not depend on $r$. Hence $M^{*}(x, r) \leq C^{\prime} r^{q / p} N(x, a)$ and we can apply Theorem 4.10 .

The equivalence of (4) and (5) is proved in Theorem 4.7. We have seen that (1) implies (2) and (2) implies (3) (Lemma 5.1). We also have that (5) implies (1) by Theorem 4.8. It remains to show that (3) implies (5). For this by Theorem 4.8 we need only establish the infinitesimal inequality (4.9). To this end, if $B$ is any ball, note that if $0 \leq f \in L^{p}(\sigma)$ and $K f \geq \chi_{B}$, then

$$
|B|_{\omega} \leq \int \chi_{B} K f d \omega=\int\left(K \omega_{B}\right) f d \sigma .
$$

Hence

$$
|B|_{\omega} \leq C^{1 / q}|B|_{\omega}^{1 / q}\|f\|_{p}
$$

by the testing condition. Thus

$$
|B|_{\omega} \leq C_{1}\|f\|_{p}^{p}
$$

for a suitable $C_{1}$. However we then deduce that

$$
|B|_{\omega} \leq C_{1} \text { Cap } B
$$


Hence

$$
\begin{aligned}
\int_{a}^{\infty} \frac{\left|B_{t}(x)\right|_{\omega}}{t^{2}} d t & \leq C_{1} \int_{a}^{\infty} \frac{\operatorname{Cap} B_{t}(x)}{t^{2}} d t \\
& \leq C_{2} \int_{a}^{\infty} \frac{N(x, t)^{-p / q}}{t^{2}} d t \\
& \leq C_{3} M(x, a)^{-p / q}
\end{aligned}
$$

by Theorem 5.4 and Lemma 5.5. But this implies that

$$
\left(\int_{0}^{a} \frac{\left|B_{t}(x)\right|_{\sigma}}{t^{2}} d t\right)^{p / q}\left(\int_{a}^{\infty} \frac{\left|B_{t}(x)\right|_{\omega}}{t^{2}} d t\right) \leq C_{4}
$$

i.e. the infinitesimal inequality holds.

Let us remark at this point that (5.11) can be equivalently formulated as

$$
M(x, a) \leq C\left|B_{a}(x)\right|_{\sigma}^{1 / p} N(x, a)^{1 / q} .
$$

That (5.12) implies (5.11) follows from the fact that $\left|B_{a}(x)\right|_{\sigma} \leq q a^{q} N(x, a)$. Now assume (5.11), and let $\theta=a M(x, a) / \alpha\left|B_{a}(x)\right|_{\sigma}$, where $\alpha=\max (2,4 C / q)$. Then

$$
M\left(x, \theta^{-1} a\right) \geq M(x, a)-(\theta-1) a^{-1}\left|B_{a}(x)\right|_{\sigma} \geq \frac{1}{2} M(x, a) .
$$

Now

$$
\begin{aligned}
M\left(x, \theta^{-1} a\right) & \leq C \theta^{-q+1} a^{q-1} N\left(x, \theta^{-1} a\right) \\
& \leq C \theta^{-q+1} a^{q-1} N(x, a)+\frac{C}{q} \theta a^{-1}\left|B_{a}(x)\right|_{\sigma} \\
& \leq C \alpha^{q-1} M(x, a)^{1-q}\left|B_{a}(x)\right|_{\sigma}^{q-1} N(x, a)+\frac{C}{q \alpha} M(x, a) \\
& \leq C \alpha^{q-1} M(x, a)^{1-q}\left|B_{a}(x)\right|_{\sigma}^{q-1} N(x, a)+\frac{1}{4} M(x, a) .
\end{aligned}
$$

Thus

$$
M(x, a) \leq 4 C \alpha^{q-1} M(x, a)^{1-q}\left|B_{a}(x)\right|_{\sigma}^{q-1} N(x, a)
$$

which can be reorganized as (5.12).

Theorem 5.7. Under the same hypotheses as in Theorem 5.6, we have $\mathcal{Y}_{p, K}=$ $\mathcal{Z}_{q, K}$ or, equivalently, the following conditions on $f \in L_{+}^{0}$ are equivalent:

(1) There exists $\epsilon>0$ so that the equation $u=K u^{q}+\epsilon f$ has a solution $u \in L_{+}^{0}$.

(2) There is a constant $C$ so that

$$
\int(K g)^{p} f^{q} d \sigma \leq C \int g^{p} d \sigma
$$

for all $g \in L^{p}(\sigma)$.

Proof. Just observe that $f \in \mathcal{Z}$ if and only if $K f^{q} \in \mathcal{Z}$ and apply the equivalence of (1) and (4) in Theorem 5.6.

We now turn to the problem of converses. We will show that under mild conditions (5.11) is necessary for the equivalences of (1) and (4) of Theorem 5.6 or of (1) and (2) in Theorem 5.7. 
Theorem 5.8. (1) Let $K$ be a quasi-metric kernel and suppose there is a stable measure $\mu$ for $K$ which is $\sigma$-continuous. Then if $\mathcal{Y}_{p, K}=\mathcal{Z}_{q, K} \neq\{0\}$ (i.e. (1) and (2) of Theorem 5.7 are equivalent), then (5.11) holds i.e. for some $C$ we have $M(x, a) \leq C a^{q-1} N(x, a)$ for all $x \in X$ and $a>0$.

(2) Suppose $K$ is a continuous quasi-metric kernel such that each ball $B_{a}(x)$ is compact. Suppose that there is a stable measure $\mu$ for $K$. Then if for every measure $\omega \in \widetilde{\mathcal{W}}_{p, K}$ we have $K \omega \in \mathcal{Z}_{q, K}$, then (5.11) holds.

Proof. (1) The hypotheses imply that $\mathcal{Y}_{p, K}=\mathcal{Z}_{q, K}$ are Banach function spaces with equivalent norms. Hence for some constant $C$ and any Borel set $E$ we have

$$
\left\|\chi_{E}\right\|_{\mathcal{Z}} \leq C\left\|_{E}\right\|_{\mathcal{Y}}=\left\|\chi_{E}\right\|_{\mathcal{W}}^{1 / q} \leq|E|_{\sigma}^{1 / q}\left\|\chi_{E}\right\|_{\mathcal{W}^{\prime}}^{-1 / q}=|E|_{\sigma}^{1 / q}(\operatorname{cap} E)^{-1 / q}
$$

If we apply this to a ball $B_{a}(x)$ using Theorem 3.8 , we obtain

$$
M(x, a) \leq C_{1}\left|B_{a}(x)\right|_{\sigma}^{1 / p}\left(\operatorname{cap} B_{a}(x)\right)^{-1 / p}
$$

and hence by Theorem 5.4

$$
M(x, a) \leq C_{2}\left|B_{a}(x)\right|_{\sigma}^{1 / p} N(x, a)^{1 / q} \leq C_{3} a^{q-1} N(x, a) .
$$

(2) The hypotheses can easily be seen to imply the existence of a constant $C$ so that if $\omega$ is any Borel measure such that

$$
\int(K f)^{p} d \omega \leq \int f^{p} d \sigma
$$

for all $f \in L^{p}$, then the infinitesimal inequality (3.9) holds with constant $C$, i.e.

$$
\sup _{a>0}\left\{\int_{0}^{a} \frac{\left|B_{t}(x)\right|_{\sigma}}{t^{2}} d t\right\}^{p / q}\left\{\int_{a}^{\infty} \frac{\left|B_{t}(x)\right|_{\omega}}{t^{2}} d t\right\} \leq C .
$$

Now by Proposition 5.3, we can find a measure $\omega$ supported on $B_{a}(x)$ with

$$
\left|B_{a}(x)\right|_{\omega}=\operatorname{Cap} B_{a}(x)
$$

and so that (5.12) holds. Then we have

$$
M(x, a)^{p / q} \text { Cap } B_{a}(x) \leq C_{1} .
$$

Again appealing to Theorem 5.4 gives the result.

It may happen that the equivalence of (1) and (4) of Theorem 5.6 or (1) and (2) of Theorem 5.7 hold even when condition (5.11) fails, however. Of course this can only happen if there is no stable measure for $K$.

Theorem 5.9. Suppose $K$ is a quasi-metric kernel and that $\sigma$ satisfies the conditions (5.13) and (5.14) for some constant $C$ :

$$
M(x, 2 a) \leq C M(x, a)
$$

and

$$
M(y, a) \leq C M(x, a)
$$

whenever $x, y \in X$ and $\rho(x, y) \leq a$. Suppose also that $N(x, a)<\infty$ for some $x \in X$ and $a>0$. Suppose $\omega \in \mathcal{M}_{+}(X)$. Then $\mathcal{Z}_{q, K} \neq\{0\}$, and the following conditions are equivalent:

(1) $\omega \in \widetilde{\mathcal{W}}_{p, K}$, i.e. $\omega$ satisfies the weighted norm inequality

$$
\int|K g|^{p} d \omega \leq C \int|g|^{p} d \sigma
$$


for all $g \in L^{p}(\sigma)$.

(2) $\omega$ satisfies the capacity condition $|E|_{\omega} \leq$ Cap $E$ for all Borel sets B. (Equivalently the weak-type inequality (5.4) holds.)

(3) $\omega$ satisfies the testing condition (4.8), i.e.

$$
\int\left(K \omega_{B}\right)^{q} d \sigma \leq C|B|_{\omega}
$$

for all balls $B$.

(4) $K \omega \in \mathcal{Z}_{q, K}$, i.e. for some $\epsilon>0$ there is a solution $u$ of the equation $u=K u^{q}+\epsilon K \omega$.

(5) $K \omega \in L_{+}^{0}(\sigma)$ and there is a constant $C$ so that $K(K \omega)^{q} \leq C K \omega$.

Remark. We remark that (5.13) is equivalent to the requirement that we have an estimate $\left|B_{2 a}(x)\right|_{\sigma} \leq C a M(x, a)$ since $M(x, 2 a) \leq(2 a)^{-1}\left|B_{2 a}(x)\right|_{\sigma}+M(x, a) \leq$ $2 M(x, 4 a)$. In particular it is sufficient that we have a doubling estimate $\left|B_{2 a}(x)\right|_{\sigma} \leq$ $C\left|B_{a}(x)\right|_{\sigma}$ for any constant $C$.

Proof. We first establish $\mathcal{Z}_{q, K} \neq\{0\}$. Fix $a>0$ and $x \in X$ so that $N(x, a)<\infty$. Then for $r>a$ we have an estimate $\left|B_{r}(x)\right|_{\sigma} \leq C r^{q}$. Now

$$
M(x, r)=M(x, a)+\int_{a}^{r} \frac{\left|B_{t}(x)\right|}{t^{2}} d t \leq M(x, a)+C^{\prime} r^{q-1} .
$$

Hence by (5.14)

$$
M^{*}(x, r) \leq M(x, a)+C^{\prime} r^{q-1} .
$$

Since $1-q=-q / p$, we have $\sup _{r>a} r^{-q / p} M^{*}(x, r)<\infty$, and Theorem 4.10 applies.

To complete the proof we argue as in Theorem 5.6. It is only necessary to show that if (3) holds, then the infinitesimal inequality (4.9) holds. We note as in Theorem 5.6 that (3) implies the capacity condition

$$
|B|_{\omega} \leq C \text { Cap } B
$$

for any ball $B$.

Let us estimate $\operatorname{Cap} B_{a}(x)$. In fact $K \chi_{B_{2 \kappa a}(x)}(y) \geq M(y, a)$ if $y \in B_{a}(x)$ so that we obtain an estimate

$$
\text { Cap } B_{a}(x) \leq C_{1} M(x, a)^{-p}\left|B_{2 \kappa a}(x)\right|_{\sigma} .
$$

Thus we have

$$
\int_{a}^{\infty} \frac{\left|B_{t}(x)\right|_{\omega}}{t^{2}} d t \leq C_{2} \int_{a}^{\infty} M(x, t)^{-p} \frac{\left|B_{2 \kappa t}(x)\right|_{\sigma}}{t^{2}} d t
$$

Now we obviously have an estimate $M(x, 2 \kappa t) \leq C_{3} M(x, t)$ so that upon substituting $\tau=2 \kappa t$ we can obtain

$$
\int_{a}^{\infty} \frac{\left|B_{t}(x)\right|_{\omega}}{t^{2}} d t \leq C_{4} \int_{2 \kappa a}^{\infty} M(x, \tau)^{-p} \frac{\left|B_{\tau}(x)\right|_{\sigma}}{t^{2}} d t=C_{4}(p-1)^{-1} M(x, 2 \kappa a)^{1-p} .
$$

Using the estimate (5.14) again this gives us the infinitesimal condition (4.9). 


\section{Trace inequalities, Carleson measure theorems, AND NONLINEAR CONVOLUTION EQUATIONS}

In this section we give some applications of the results of the previous section to trace inequalities for Riesz potentials as well as more general convolution operators on $\mathbf{R}^{n}$, and the solvability problem for the corresponding nonlinear convolution equations. We also obtain a weighted version of the Carleson measure theorem for Poisson integrals on $\mathbf{R}_{+}^{n+1}$ which generalizes a result of Treil and Volberg [50] where the case $p=2$ was considered. (The proof in [50] makes use of a test for boundedness of quadratic forms; in the classical unweighted case this idea is due to S.A. Vinogradov [40].) Our approach is closer to the well-known proof of Hardy's inequality (see e.g. [34]) and works for all $1<p<\infty$.

Theorem 6.1. Let $k: \mathbf{R}^{n} \rightarrow \mathbf{R}$ be a positive radial function of the form $k(x)=$ $h(\|x\|)$ for $x \neq\{0\}$ where $h$ is decreasing and

$$
\inf _{r>0} \frac{h(2 r)}{h(r)}>0 .
$$

Suppose $k \in L^{1}+L^{q}$. Let $\lambda$ be Lebesgue measure on $\mathbf{R}^{n}$ and suppose $\omega$ is any locally finite Borel measure. Then the following are equivalent:

(1) There exists $C$ so that we have

$$
\int(k * f)^{p} d \omega \leq C \int f^{p} d \lambda
$$

for all $f \geq 0$.

(2) $k * \omega \in L_{+}^{0}$ and

$$
k *(k * \omega)^{q} \leq C^{\prime} k * \omega
$$

for some constant $C^{\prime}$.

(3) $k * \omega \in \mathcal{Z}$, or equivalently the equation $u=k * u^{q}+\epsilon(k * \omega)$ has a solution for all sufficiently small $\epsilon>0$.

(4) The testing inequality

$$
\int_{B}\left(k * \omega_{B}\right)^{q} d \lambda \leq C|B|_{\omega}
$$

holds for all Euclidean balls $B$ in $\mathbf{R}^{n}$.

(5) There exists $C$ so that

$$
|E|_{\omega} \leq C \operatorname{Cap}_{p, k}(E)
$$

for all compact sets $E$.

Remarks. (a) Characterizations of trace inequalities in terms of capacities are due to V. Maz'ya, D. Adams, and B. Dahlberg in the case of Riesz potentials (see [3], [35]). For radial kernels similar characterizations in terms of capacity inequalities or testing inequalities with totally different and more difficult proofs are due to K. Hansson [21] and E. Sawyer and R. Kerman [27].

(b) A characterization of solvability for the equation in (3) with an arbitrary inhomogeneous term $f \geq 0$ in place of $k * \omega$ is given by $k *\left(k * f^{q}\right)^{q} \leq C k * f^{q}$.

(c) We remark that we make use of Theorem 5.9 and not 5.6 which would only apply to special cases of such convolution operators. 
Proof. If $K(x, y)=k(x-y)$, then $K$ satisfies the quasi-metric assumption. The assumptions on $h$ ensure that $M(x, a)$ and $N(x, a)$ are everywhere finite for $\sigma=\lambda$. In this case $M(x, a)$ is constant for each $a$. Note that in this case $B_{a}(0)=\{x$ : $\left.h(\|x\|) \geq a^{-1}\right\}$ and so we have an estimate that $B_{2 a}(0) \subset C B_{a}(0)$ which implies $\left|B_{2 a}(0)\right|_{\lambda} \leq C^{n}\left|B_{a}(0)\right|_{\lambda}$. By the remarks following Theorem 5.9, this means we can apply this theorem to yield the result. Note that in (5) one can use compact sets in place of Borel sets due to the known capacitability results (see [3], p. 28).

Next we characterize trace inequalities for Riesz potentials of order $\alpha, I_{\alpha}=$ $(-\Delta)^{-\alpha / 2}$, on $\mathbf{R}^{n}$, and the solvability problem for the integral equation

$$
u=I_{\alpha}\left(u^{q} d \sigma\right)+f, \quad 0 \leq u<\infty \quad d \sigma \text {-a.e. }
$$

Note that in contrast to Theorem 6.1 now $\sigma$ is not necessarily Lebesgue measure.

We will make use of the class of $A_{\infty}^{\beta}$-weights introduced in [45], which contains both Muckenhoupt $A_{\infty}$-weights (in case $\beta=n$ ) and (reverse) doubling weights $R D_{\beta}$ such that

$$
\left|B_{r}\right|_{\sigma} \leq C\left(\frac{r}{R}\right)^{\beta}\left|B_{R}\right|_{\sigma}
$$

for all concentric balls $B_{r}$ and $B_{R}$ with $0<r<R<\infty$. We set $\mathcal{Z}_{q, \alpha}=\mathcal{Z}_{q, I_{\alpha}}$ and $S_{q, \alpha}=S_{q, I_{\alpha}}$. We also set

$$
I_{\alpha}^{\sigma} f(x)=I_{\alpha}(f d \sigma)(x)=C(n, \alpha) \int \frac{f(y) d \sigma}{|x-y|^{n-\alpha}},
$$

where $C(n, \alpha)=\pi^{-n / 2} 2^{-\alpha} \Gamma(n / 2-\alpha / 2) \Gamma(\alpha / 2)^{-1}$.

Theorem 6.2. Let $1<q<\infty$ and $0<\alpha<n$. Let $\sigma$ and $\omega$ be locally finite measures on $\mathbf{R}^{n}$, and let $f=I_{\alpha} \omega \in L_{+}^{0}(\sigma)$. Then the following statements are true.

(1) $f \in \mathcal{Z}_{q, \alpha}$ if and only if the inequality

$$
I_{\alpha}^{\sigma}\left(I_{\alpha} \omega\right)^{q} \leq C I_{\alpha} \omega \quad d \sigma \text {-a.e. }
$$

holds. Moreover, if (6.2) holds with $C=p^{1-q} q^{-1}$, then $f \in S_{q, \alpha}$, and (6.1) has a solution $u$ such that $I_{\alpha} \omega \leq u \leq p I_{\alpha} \omega$.

(2) $f \in \mathcal{Z}_{q, \alpha}$ if and only if both the trace inequality

$$
\left\|I_{\alpha}^{\sigma} h\right\|_{L^{p}(\omega)} \leq C\|h\|_{L^{p}(\sigma)}, \quad h \in L^{p}(\sigma)
$$

and the infinitesimal inequality

$$
\sup _{x \in \mathbf{R}^{n}, r>0}\left\{\int_{0}^{r} \frac{\left|B_{t}(x)\right|_{\sigma}}{t^{n-\alpha+1}} d t\right\}^{1 / q}\left\{\int_{r}^{\infty} \frac{\left|B_{t}(x)\right|_{\omega}}{t^{n-\alpha+1}} d t\right\}^{1 / p}<\infty
$$

hold, where $B_{r}(x)$ is a Euclidean ball of radius $r$ centered at $x$.

(3) $f \in \mathcal{Z}_{q, \alpha}$ if and only if both the infinitesimal inequality (6.4) and the testing inequality

$$
\int_{B}\left(I_{\alpha}^{\omega} \chi_{B}\right)^{q} d \sigma \leq C|B|_{\omega}
$$

hold, where $C$ is independent of $B=B_{r}(x)$.

(4) If $\sigma$ satisfies the estimate

$$
\int_{0}^{r} \frac{\left|B_{t}(x)\right|_{\sigma}}{t^{n-\alpha+1}} d t \leq C r^{(n-\alpha)(q-1)} \int_{r}^{\infty} \frac{\left|B_{t}(x)\right|_{\sigma}}{t^{(n-\alpha) q+1}} d t
$$


then $(6.3) \Leftrightarrow(6.5) \Leftrightarrow(6.2)$. Moreover, (6.6) is necessary in order that (6.3) $\Leftrightarrow(6.2)$.

(5) If $\sigma, \omega \in A_{\infty}^{\beta}$ with $\beta>n-\alpha$, then (6.2) is equivalent to the infinitesimal inequality (6.4).

(6) If $\sigma, \omega \in R D_{\beta}$ with $\beta>n-\alpha$, then (6.2) is equivalent to the following condition of Muckenhoupt type:

$$
\sup _{x \in \mathbf{R}^{n}, r>0} \frac{\left|B_{r}(x)\right|_{\sigma}^{1 / q}\left|B_{r}(x)\right|_{\omega}^{1 / p}}{r^{n-\alpha}}<\infty .
$$

Remarks. (a) For $\sigma \in R D_{\beta}$ with $\beta>n-\alpha$ similar results were proved earlier in [52]. Note that in this case (6.6) holds and hence (6.4) follows from (6.5) which simplifies the proofs.

(b) There are analogous criteria of solvability of (6.1) for an arbitrary $f \in L_{+}^{0}$ in place of $I_{\alpha} \omega$ (see Theorem 4.9). In particular, $f \in \mathcal{Z}_{q, \alpha}$ if and only if

$$
I_{\alpha}^{\sigma}\left(I_{\alpha}^{\sigma} f^{q}\right)^{q} \leq C I_{\alpha}^{\sigma} f^{q} .
$$

Moreover, if (6.8) holds with $C=p^{q(1-q)} q^{-q}$, then $f \in S_{q, \alpha}$ i.e. (6.1) has a solution $u$ such that

$$
f+I_{\alpha}^{\sigma} f^{q} \leq u \leq f+p^{q} I_{\alpha}^{\sigma} f^{q} .
$$

Proof. The proofs of statements (1)-(4) of the theorem follow easily from Theorems 4.8, 5.6, and 5.8. One only need notice that the balls $B_{r}(x)$ associated with the Riesz metric $\rho(x, y)=C(n, \alpha)^{-1}|x-y|^{n-\alpha}$ correspond to Euclidean balls with the same center and radius $C(n, \alpha)^{1 /(n-\alpha)} r^{1 /(n-\alpha)}$.

Under the assumptions of statement (5) of the theorem the weighted norm inequality (6.3) is equivalent to (6.7) by Theorem 2 of [45]. To prove (5), note that obviously $(6.4) \Rightarrow(6.7)$, and apply $(2)$.

To prove statement (6), observe that since $\sigma \in R D_{\beta}$, then clearly (6.6) holds. Hence by $(4)(6.2) \Leftrightarrow(6.5)$. It is also well known that in this case (6.7) is necessary and sufficient in order that the weighted norm inequality (6.3) hold (see [41], [45]). Thus $(6.5) \Leftrightarrow(6.7)$ which proves $(6)$.

We now give an application to weighted Carleson measure inequalities for Poisson integrals. Let $\omega$ and $\sigma$ be locally finite Borel measures on $\mathbf{R}_{+}^{n+1}=\mathbf{R}^{n} \times \mathbf{R}_{+}^{n}$ and $\mathbf{R}^{n}$ respectively. Let $1<p<\infty$. We consider the inequality

$$
\left\|P^{\sigma} f\right\|_{L^{p}(\omega)} \leq C\|f\|_{L^{p}(\sigma)}
$$

for the Poisson integral

$$
P^{\sigma} f(x, t)=P[f d \sigma](x, t)=\int_{\mathbf{R}^{n}} P_{t}(x-y) f(y) d \sigma(y) .
$$

Here $(x, t) \in \mathbf{R}_{+}^{n+1}$ and $P_{t}(y)=C_{n} t /\left(\|y\|^{2}+t^{2}\right)^{(n+1) / 2}$ is the Poisson kernel on the upper half-space. Similarly, for $\omega \in M_{+}\left(\mathbf{R}_{+}^{n+1}\right)$ we set

$$
P^{\omega} g(x, t)=\int_{\mathbf{R}_{+}^{n+1}} P_{t+\tau}(x-y) g(y, \tau) d \omega(y, \tau) .
$$

If $\sigma$ is Lebesgue measure, then the Carleson measure theorem [10] says that (6.9) holds if and only if $\omega$ is a Carleson measure, i.e. $|\widehat{B}|_{\omega} \leq C|B|$; here $\widehat{B}$ is the 
cylinder with height $|B|^{1 / n}$ whose base is a ball $B$ in $\mathbf{R}^{n}$. It is easy to see that $\omega$ is a Carleson measure if and only if

$$
P^{\omega} \mathbf{1}(x, t)=\int_{\mathbf{R}_{+}^{n+1}} P_{t+\tau}(x-y) d \omega(y, \tau) \leq C<\infty
$$

where $C$ is independent of $(x, t) \in \mathbf{R}_{+}^{n+1}$. (See [19], [40].) A simple proof of the nontrivial implication $(6.10) \Rightarrow(6.9)$ (in case $\sigma$ is Lebesgue measure) is immediate from the following theorem.

Theorem 6.3. Let $1<p<\infty$ and let $\omega \in M_{+}\left(\mathbf{R}_{+}^{n+1}\right), \sigma \in M_{+}\left(\mathbf{R}^{n}\right)$. Then (6.9) holds if $P^{\sigma} \mathbf{1} \not \equiv+\infty$ and

$$
P^{\omega}\left(P^{\sigma} \mathbf{1}\right)^{p}(x, t) \leq C P^{\sigma} \mathbf{1}(x, t) .
$$

The case $p=2$ of Theorem 6.3 was established in [50]. Note that the pointwise condition (6.11) is used in a dual form so that if $\sigma$ is Lebesgue measure we have $P^{\sigma} \mathbf{1}=\mathbf{1}$; then (6.11) coincides with (6.10).

Proof. Consider $d \omega_{1}(x, t)=t d \omega(x, t)$ and $d \sigma_{1}=\chi_{\mathbf{R}^{n}} d \sigma$ as measures on $X=\overline{\mathbf{R}_{+}^{n+1}}$. Let $K$ be a quasi-metric kernel on $X \times X$ defined by

$$
K(\bar{x}, \bar{y})=\left[\|x-y\|^{2}+(t+\tau)^{2}\right]^{-(n+1) / 2}
$$

where $\bar{x}=(x, t)$ and $\bar{y}=(y, \tau)$.

It is easy to see that (6.11) is equivalent to

$$
K^{\omega_{1}}\left(K^{\sigma_{1}} \mathbf{1}\right)^{p} \leq C K^{\sigma_{1}} \mathbf{1}
$$

Then by Theorem 4.8 applied to $L^{q}\left(\omega_{1}\right)$ in place of $L^{p}(\sigma)$ it follows that

$$
\left\|K^{\omega_{1}} g\right\|_{L^{q}\left(\sigma_{1}\right)} \leq C\|g\|_{L^{q}\left(\omega_{1}\right)}
$$

for all $g \in L^{q}\left(\omega_{1}\right)$, which is obviously equivalent to the inequality dual to (6.9). Note that here we have only used an easy part of Theorem 4.8 which is essentially contained in the elementary estimates of Propositions 4.4 and 4.5 .

\section{EXISTENCE OF POSITIVE SOLUTIONS FOR SUPERLINEAR DIRICHLET PROBLEMS}

In this section, we obtain some estimates for the Green kernels and Naïm kernels related to the so-called $3 G$-inequalities. This makes it possible, as an application of the results of Sections $2-5$, to characterize the problem of the existence of positive solutions for the superlinear Dirichlet problem

$$
\left\{\begin{array}{l}
-\Delta u=v(x) u^{q}+w(x), \quad u \geq 0 \quad \text { on } \Omega, \\
u=\phi \text { on } \partial \Omega,
\end{array}\right.
$$

on a regular domain $\Omega \subset \mathbf{R}^{n}$ in the "superlinear case" $q>1$; here we assume that $v, w \in L_{l o c}^{1}(\Omega)$ and $\phi \in L_{l o c}^{1}(\partial \Omega)$ are arbitrary nonnegative functions. We denote by $G=G_{\Delta, \Omega}$ the Green function of the Laplacian $\Delta$ on $\Omega$, and by $G u$ the Green potential

$$
G u(x)=\int_{\Omega} G(x, y) u(y) d y
$$


By $P \phi$ we denote the Poisson integral (harmonic extension) of $\phi$. The solvability of (7.1) is understood in the sense (see [6], [29]) that $u \in L_{l o c}^{q}(\Omega)$ satisfies the corresponding nonlinear integral equation

$$
u=G\left(v u^{q}\right)+G w+P \phi \quad \text { a.e. on } \Omega .
$$

More generally, we consider the Dirichlet problem

$$
\left\{\begin{array}{l}
-L u=\sigma u^{q}+\omega, \quad u \geq 0 \text { on } \Omega, \\
u=\phi \text { on } \partial \Omega,
\end{array}\right.
$$

where $\sigma, \omega$ are locally finite measures on $\Omega$ (possibly singular with respect to Lebesgue measure), and $L$ is a uniformly elliptic differential operator of second order,

$$
L u=\sum_{i, j=1}^{n} a_{i j}(x) D_{i j} u+\sum_{i=1}^{n} b_{i}(x) D_{i} u+c(x) u,
$$

with the assumptions on the coefficients and $\Omega$ specified below. (See Proposition 7.2 and Lemma 7.1.) Let $G=G_{L, \Omega}$ be the Green function of $L$. We say that $u \in L_{l o c}^{q}(d \sigma, \Omega)$ is a solution to $\left(7.1^{\prime}\right)$ if $u$ satisfies the integral equation

$$
u=G^{\sigma} u^{q}+G^{\omega} \mathbf{1}+P \phi \quad d \sigma \text {-a.e. on } \Omega .
$$

Here $G^{\nu} u(x)$ is the Green potential with respect to a locally finite measure $u d \nu$ defined by

$$
G^{\nu} u(x)=\int_{\Omega} G(x, y) u(y) d \nu(y),
$$

and $P \phi=P_{L, \Omega} \phi$ is the corresponding Poisson integral ( $L$-harmonic extension of $\phi)$.

Our assumptions on $L$ and $\Omega$ will be stated in terms of certain inequalities for the Green function $G=G_{L, \Omega}$. As was mentioned in the Introduction, $G$ does not satisfy our basic quasi-metric assumptions even in the case of the Laplacian on a ball or half-space. In the so-called $3 G$-inequality (see [12], [13]),

$$
\frac{G(x, y) G(y, z)}{G(x, z)} \leq \kappa\left(|x-y|^{2-n}+|y-z|^{2-n}\right)
$$

unfortunately, one cannot replace the right-hand side by a smaller term $G(x, y)+$ $G(y, z)$.

However, we can reduce the solvability problem for $\left(7.2^{\prime}\right)$ to the problems studied above by using the so-called Naïm kernel. For $x \in \Omega$, we denote by $\delta(x)$ the distance from $x$ to the boundary $\partial \Omega$. If $\partial \Omega$ is smooth enough, we set

$$
N(x, y)=\frac{G(x, y)}{\delta(x) \delta(y)} .
$$

Then we will show that $N$ satisfies the inequality

$$
\frac{N(x, y) N(y, z)}{N(x, z)} \leq \kappa[N(x, y)+N(y, z)],
$$

which is obviously equivalent to our quasi-metric assumption. It is worthwhile to note that (7.6) is always stronger than the original $3 G$-inequality (7.4), and takes into account the behavior of the Green kernel at the boundary in a proper way. (See [49] for another refinement of (7.4) in a different direction, which is, however, again not sharp at the boundary.) 
We observe that this approach is applicable at least to operators $L$ with Höldercontinuous coefficients on bounded $C^{1,1}$ domains $\Omega$. For more general domains, as was proposed in [38], one may replace the distance to the boundary $\delta(x)$ in the definition (7.5) by $s(x)=G\left(x, x_{0}\right)$, where $x_{0}$ is a fixed pole in $\Omega$. We conjecture that in this setting (7.6) holds, with obvious modifications, for bounded Lipschitz domains $\Omega$ and operators $L$ with bounded measurable coefficients.

The proof of (7.6) under the assumptions stated above is based on the well known two-sided estimates

$$
G_{L, \Omega}(x, y) \asymp|x-y|^{2-n} \min \left[1, \frac{\delta(x) \delta(y)}{|x-y|^{2}}\right]
$$

for the Green function $G_{L, \Omega}$. The upper estimate in (7.7) is due to K.-O. Widman [55] for $C^{1, \alpha}$ (or more general Dini type) domains, and the lower one was established by Z. Zhao [56] for $C^{1,1}$ domains and $L=\Delta$. For second order uniformly elliptic operators $L$ with Hölder-continuous coefficients, it was proved by Hueber and Sieveking [25] that $G_{L, \Omega} \asymp G_{\Delta, \Omega}$. (See also [5], [49], and the references given there.)

Lemma 7.1. Let $\Omega$ be a bounded $C^{1,1}$ domain in $\mathbf{R}^{n}, n \geq 3$. Let $L$ be a uniformly elliptic second order differential operator (7.3) with bounded Hölder-continuous coefficients, and $c \leq 0$. Then $\rho(x, y)=N(x, y)^{-1}$ defines a quasi-metric on $\Omega$, and thus (7.6) holds.

Remark. In Lemma 7.1, one can replace the restrictions on $c$ and $b_{i}$ by some milder assumptions which guarantee that $G_{L, \Omega} \asymp G_{\Delta, \Omega}$ (see [5], [12], [13]). Analogues of Lemma 7.1 also hold in the cases $n=1,2$ which require usual modifications.

Proof. We first show that (7.7) is equivalent to the estimate

$$
G_{L, \Omega}(x, y) \asymp \frac{\delta(x) \delta(y)}{|x-y|^{n-2}\left[|x-y|^{2}+\delta(x)^{2}+\delta(y)^{2}\right]} .
$$

We have

$$
\begin{gathered}
\min \left[1, \frac{\delta(x) \delta(y)}{|x-y|^{2}}\right]=\frac{1}{\max \left[1, \frac{|x-y|^{2}}{\delta(x) \delta(y)}\right]} \\
\asymp \frac{\delta(x) \delta(y)}{|x-y|^{2}+\delta(x) \delta(y)} .
\end{gathered}
$$

It remains to notice that from the obvious inequality $|\delta(x)-\delta(y)| \leq|x-y|$ it follows

$$
|x-y|^{2}+\delta(x) \delta(y) \asymp|x-y|^{2}+\delta(x)^{2}+\delta(y)^{2} .
$$

Hence $\left(7.7^{\prime}\right)$ holds. Now for the Naïm kernel defined by (7.5) we have

We set

$$
N(x, y) \asymp \frac{1}{|x-y|^{n-2}\left[|x-y|^{2}+\delta(x)^{2}+\delta(y)^{2}\right]} .
$$

$$
d(x, y)=|x-y|^{n-2}\left[|x-y|^{2}+\delta(x)^{2}+\delta(y)^{2}\right],
$$

where $d(x, y) \asymp N(x, y)^{-1}$. Then to prove (7.6) it suffices to show that $d(x, y)$ satisfies the quasi-metric inequality

$$
d(x, y) \leq C[d(x, z)+d(y, z)] .
$$


We notice

$$
d(x, y)=|x-y|^{n}+\delta(x)^{2}|x-y|^{n-2}+\delta(y)^{2}|x-y|^{n-2} .
$$

Estimating the first term on the right from above, we obviously have

$$
|x-y|^{n} \leq 2^{n-1}\left[|x-z|^{n}+|y-z|^{n}\right] \leq 2^{n-1}[d(x, z)+d(y, z)] .
$$

It remains to estimate $\delta(x)^{2}|x-y|^{n-2}$, since a similar bound for $\delta(y)^{2}|x-y|^{n-2}$ follows by interchanging the roles of $x$ and $y$.

To prove the inequality

$$
\delta(x)^{2}|x-y|^{n-2} \leq C[d(x, z)+d(y, z)],
$$

we consider two cases, (i) $\delta(x) \leq \delta(y)$ and (ii) $\delta(y) \leq \delta(x)$.

In case (i), clearly,

$$
\begin{gathered}
\delta(x)^{2}|x-y|^{n-2} \leq 2^{n-3} \delta(x)^{2}\left[|x-z|^{n-2}+|y-z|^{n-2}\right] \\
\leq 2^{n-3} \delta(x)^{2}|x-z|^{n-2}+2^{n-3} \delta(y)^{2}|y-z|^{n-2} \leq 2^{n-3}[d(x, z)+d(y, z)],
\end{gathered}
$$

which implies (7.8).

In case (ii), use the inequality $\delta(x) \leq \delta(y)+|x-y|$. Then we get

$$
\delta(x)^{2}|x-y|^{n-2} \leq 2|x-y|^{n}+2 \delta(y)^{2}|x-y|^{n-2} .
$$

As above, for the first term on the right-hand side of the preceding inequality we have $|x-y|^{n} \leq 2^{n-1}[d(x, z)+d(y, z)]$. Estimating the second term, we have

$$
\begin{gathered}
\delta(y)^{2}|x-y|^{n-2} \leq 2^{n-3}\left[\delta(y)^{2}|x-z|^{n-2}+\delta(y)^{2}|y-z|^{n-2}\right] \\
\leq 2^{n-3}\left[\delta(x)^{2}|x-z|^{n-2}+\delta(y)^{2}|y-z|^{n-2}\right] \leq 2^{n-3}[d(x, z)+d(y, z)] .
\end{gathered}
$$

This proves (7.8) in case (ii). Thus, (7.8) holds, which implies (7.6). The proof of Lemma 7.1 is complete.

Next, we show that the main results of Sections 3-5 hold true in a more general setting where the kernel $G(x, y)$ is not necessarily symmetric and may fail to satisfy the quasi-metric inequality. We assume that there exist positive measurable functions $s_{1}$ and $s_{2}$ such that

$$
K(x, y) \asymp s_{1}(x) G(x, y) s_{2}(y), \quad x, y \in X,
$$

where $K(x, y)$ is symmetric and satisfies the quasi-metric inequality. It follows that this is the case for the Green function $G(x, y)=G_{L, \Omega}$, under the assumptions of Lemma 7.1, if $s_{1}(x)=s_{2}(x)=\delta^{-1}(x)$; then $K$ coincides with the Naïm kernel (7.5). Recall that $f \in \mathcal{Z}_{q, G}$ if the nonlinear integral equation

$$
u=G^{\sigma} u^{q}+\epsilon f \quad d \sigma \text {-a.e. on } X
$$

has a solution for some $\epsilon>0$.

Proposition 7.2. Suppose that $G$ is a kernel on $X \times X$ such that (7.9) holds, where $s_{1}$ and $s_{2}$ are positive measurable function on $X$, and $K(x, y)$ is a symmetric quasi-metric kernel. Then $f \in \mathcal{Z}_{q, G}$ if and only if

$$
G^{\sigma}\left(G^{\sigma} f^{q}\right)^{q} \leq C G^{\sigma} f^{q}<\infty \quad \text { do-a.e. }
$$


Proof. We rewrite (7.10) in the equivalent form

$$
\tilde{u}=K^{\tilde{\sigma}} \tilde{u}^{q}+\tilde{f}
$$

where

$$
\tilde{u}=s_{1} u, \quad \tilde{f}=s_{1} f, \quad d \tilde{\sigma}=s_{2}^{-1} s_{1}^{-q} d \sigma .
$$

By Theorem 4.1, this equation has a solution $\tilde{u}$ if and only if

$$
K^{\tilde{\sigma}}\left(K^{\tilde{\sigma}} \tilde{f}^{q}\right)^{q} \leq C K^{\tilde{\sigma}} \tilde{f}^{q}<\infty \quad d \sigma \text {-a.e. }
$$

Multiplying both sides of the preceding inequality by $s_{1}^{-1}$ and using (7.9) and (7.13), we see that (7.14) is equivalent to (7.11).

Remark. One of the advantages of using pointwise characterizations (7.11) is that they are invariant under the transformation of the kernels given by (7.9). It is easy to see that all other results of Sections 3-5 have complete analogues for kernels $G$ such that $K(x, y) \asymp s_{1}(x) G(x, y) s_{2}(y)$ satisfies the quasi-metric inequality.

However, all geometric conditions should be modified because of the new quasimetric. Moreover, (7.9) leads to a change of weights in the corresponding weighted norm inequalities. Using (7.9) and (7.13) in the same manner as in the proof of Proposition 7.2 , it is easily seen that the weighted norm inequality

$$
\left\|G^{\sigma} h\right\|_{L^{p}(d \omega)} \leq C\|h\|_{L^{p}(d \sigma)}, \quad h \in L^{p}(d \sigma),
$$

for $G$ is equivalent to a similar inequality

$$
\left\|K^{\sigma_{1}} g\right\|_{L^{p}\left(d \omega_{1}\right)} \leq C\|g\|_{L^{p}\left(d \sigma_{1}\right)}, \quad g \in L^{p}\left(d \sigma_{1}\right),
$$

for $K$, where $d \sigma_{1}=s_{2}^{1-q} d \sigma$ and $d \omega_{1}=s_{1}^{-p} d \omega$.

Using these results with $s_{1}=s_{2}=\delta^{-1}$, together with the testing characterizations of weighted norm inequalities [45], we obtain the following characterization of two weight inequalities for Green's potentials.

Theorem 7.3. Let $\Omega$ and $L$ satisfy the assumptions of Lemma 7.1. For $x \in \Omega$ and $a>0$, denote by $B=B_{a}(x)$ a "ball" associated with the Naïm kernel:

$$
B_{a}(x)=\left\{y \in \Omega: G(x, y) \geq a^{-1} \delta(x) \delta(y)\right\} .
$$

Then the two weight inequality (7.15) holds for the Green potential $G^{\sigma}$ if and only if, for all balls $B=B_{a}(x)$, both

$$
\int_{\Omega}\left(G^{\sigma} \delta^{q-1} \chi_{B}\right)^{p} d \omega \leq C \int_{B} \delta^{q} d \sigma
$$

and

$$
\int_{\Omega}\left(G^{\omega} \delta^{p-1} \chi_{B}\right)^{q} d \sigma \leq C \int_{B} \delta^{p} d \omega
$$

hold.

The same argument as in Proposition 7.2, together with Theorem 4.9, yields the following characterization of the solvability problem for the nonlinear integral equation (7.10). (Note that Theorem 7.3 is not used in this proof.) 
Theorem 7.4. Let $\Omega$ be a bounded $C^{1,1}$ domain in $\mathbf{R}^{n}, n \geq 3,1<q<\infty$, and let $L$ be a uniformly elliptic second order differential operator as in Lemma 7.1. Let $f \in L_{\text {loc }}^{q}(d \sigma, \Omega)$ be a nonnegative function on $\Omega$. Then the following statements are equivalent.

(1) Equation (7.10) has a solution for some $\epsilon>0$.

(2) There exists a constant $C>0$ such that

$$
G^{\sigma}\left(G^{\sigma} f^{q}\right)^{q} \leq C G^{\sigma} f^{q}<\infty \quad \text { do-a.e. }
$$

(3) Both the weighted inequality

$$
\int_{\Omega}\left(G^{\sigma} h\right)^{p} f^{q} \delta^{1-p} d \sigma \leq C \int_{\Omega} h^{p} \delta^{1-p} d \sigma
$$

and the infinitesimal inequality

$$
\left\{\int_{0}^{a} \frac{\int_{B_{t}(x)} \delta^{1+q} d \sigma}{t^{2}} d t\right\}^{1 / q}\left\{\int_{a}^{\infty} \frac{\int_{B_{t}(x)} f^{q} \delta d \sigma}{t^{2}} d t\right\}^{1 / p} \leq C
$$

hold.

(4) Both (7.21) and the testing inequality

$$
\int_{B}\left(G^{\sigma} f^{q} \chi_{B}\right)^{q} \delta d \sigma \leq C \int_{B} f^{q} \delta d \sigma
$$

hold.

Now we are in a position to characterize the solvability of the nonlinear Dirichlet problem $\left(7.2^{\prime}\right)$. The latter is related to the integral equation (7.10) with $f=f_{1}+f_{2}$, where $f_{1}=G^{\omega} \mathbf{1}$ and $f_{2}=P \phi$. We first consider the homogeneous problem where $\phi=0$ :

$$
\left\{\begin{array}{c}
-L u=\sigma u^{q}+\epsilon \omega, \quad u \geq 0 \quad \text { on } \Omega \\
u=0 \text { on } \partial \Omega
\end{array}\right.
$$

which is equivalent to the integral equation

$$
u=G^{\sigma} u^{q}+G^{\omega} \mathbf{1} .
$$

Since in this case the inhomogeneous term of (7.24) is a Green potential, the characterizations of Theorem 7.4 may be simplified by using Theorem 4.8 in place of Theorem 4.9. This yields

Theorem 7.5. Under the assumptions of Theorem 7.4 the following statements are equivalent.

(1) The Dirichlet problem (7.23) has a solution for some $\epsilon>0$.

(2) $G \omega<\infty$ and there exists a constant $C>0$ such that

$$
G^{\sigma}\left(G^{\omega} \mathbf{1}\right)^{q} \leq C G^{\omega} \mathbf{1} .
$$

(3) Both the weighted inequality

$$
\int_{\Omega}\left(G^{\sigma} h\right)^{p} \delta^{1-p} d \omega \leq C \int_{\Omega} h^{p} \delta^{1-p} d \sigma
$$

and the infinitesimal inequality

$$
\left\{\int_{0}^{a} \frac{\int_{B_{t}(x)} \delta^{1+q} d \sigma}{t^{2}} d t\right\}^{1 / q}\left\{\int_{a}^{\infty} \frac{\int_{B_{t}(x)} \delta d \omega}{t^{2}} d t\right\}^{1 / p} \leq C
$$


hold.

(4) Both the infinitesimal and the testing inequality

$$
\int_{B}\left(G^{\omega} \chi_{B}\right)^{q} \delta d \sigma \leq C \int_{B} \delta d \omega
$$

hold.

Remark. In the one-dimensional case, we consider the problem

$$
\left\{\begin{array}{c}
-u^{\prime \prime}(x)=\sigma u(x)^{q}+\omega, \quad 0<x<1, \\
u(0)=u(1)=0 .
\end{array}\right.
$$

Here $\Omega=(0,1)$ and $G(x, y)=\min [x(1-y), y(1-x)]$.

The corresponding Naïm kernel $N(x, y)=[x y(1-x)(1-y)]^{-1} G(x, y)$ satisfies the quasi-triangle inequality with $\kappa=1$, so that $\rho(x, y)=1 / N(x, y)=$ $[1-\min (x, y)] \max (x, y)$, and thus Theorem 4.8 is applicable. Note that in the easy case where $\omega \in L^{1}(0,1)$ (or, more generally, if $\omega$ is a finite measure), we have $G \omega(x) \asymp x(1-x)$. Then (7.19) boils down to the requirement that the integral $\int_{0}^{1}[x(1-x)]^{q} d \sigma$ is finite and small enough. This is clearly necessary for the existence of solutions in case $\omega$ is a finite measure.

However, $\omega$ need not be finite on $(0,1)$ : the only natural restriction on $\omega$, which is equivalent to $G \omega<\infty$, is $\int_{0}^{1} x(1-x) d \omega<\infty$. For $\omega$ such that $\int_{0}^{1} d \omega=\infty$, the existence of positive solutions depends on the interplay between $\sigma$ and $\omega$ at the endpoints, and is determined by (7.19), or the equivalent inequalities (7.21) and (7.22). Moreover, it is not difficult to see that in this case $(7.21) \Rightarrow(7.22)$, and so the infinitesimal inequality alone characterizes the solvability problem in this case.

Now we consider the inhomogeneous Dirichlet problem with boundary data $\phi \neq$ 0 . We assume that $0 \leq P \phi<\infty$. (The estimates of the Poisson kernel for elliptic operators of second order can be found in [5], [36], [48], [56].)

It follows from Theorem 7.4 that (7.10) is solvable for some (small enough) $\epsilon>0$ if and only if both $G^{\omega} \mathbf{1}$ and $P \phi \in \mathcal{Z}_{q, G}$. Thus, applying Theorems 7.4 and 7.5 established above, we obtain the following statement.

Theorem 7.6. Under the assumptions of Theorem 7.4, the Dirichlet problem

$$
\left\{\begin{array}{c}
-L u=\sigma u^{q}+\epsilon \omega, \quad u \geq 0 \quad \text { on } \quad \Omega, \\
u=\epsilon \phi \text { on } \partial \Omega
\end{array}\right.
$$

has a solution for some $\epsilon>0$ if and only if both

$$
G^{\sigma}\left(G^{\omega} \mathbf{1}\right)^{q} \leq C G^{\omega} \mathbf{1} \quad \text { do-a.e. }
$$

and

$$
G^{\sigma}\left[G^{\sigma}(P \phi)^{q}\right]^{q} \leq C G^{\sigma}(P \phi)^{q} \quad \text { do-a.e. }
$$

hold.

Equivalent characterizations of solvability in terms of the infinitesimal inequalities and testing inequalities (or capacitary inequalities) follow as in Theorems 7.4 and 7.5 .

In conclusion we consider the special case of the nonlinear Dirichlet problem

$$
\left\{\begin{array}{l}
-L u=u^{q}+\epsilon \omega, \quad u \geq 0 \quad \text { on } \Omega, \\
u=0 \text { on } \partial \Omega,
\end{array}\right.
$$


previously characterized by D. Adams and M. Pierre [3] in the case supp $\omega \Subset \Omega$. We observe that our methods are totally different, and give additional pointwise estimates of solutions with sharp constants up to the boundary.

We introduce a weighted capacity associated with (7.25). To any $E \subset \Omega$ we associate

$$
\operatorname{Cap}_{p, G}(E)=\inf \left\{\int_{\Omega} g^{p} \delta(x)^{1-p} d x: G g(x) \geq \delta(x) \chi_{E}(x), g \geq 0\right\},
$$

where

$$
G g(x)=\int_{\Omega} G(x, y) g(y) d y
$$

is the Green potential of $g$.

Let

$$
\operatorname{Cap}_{p, I_{2}}(E)=\inf \left\{\int_{\Omega} g^{p} d x: I_{2} g(x) \geq \chi_{E}(x), g \geq 0\right\}
$$

be the nonlinear Newtonian capacity associated with the Sobolev space $W^{2, p}\left(\mathbf{R}^{n}\right)$ used in [3]. It is easily seen that for any compact set $E \Subset \Omega$ one has $\operatorname{Cap}_{p, G}(E) \asymp$ $\operatorname{Cap}_{p, I_{2}}(E)$ with constants of equivalence which depend on dist $(E, \partial \Omega)$.

Theorem 7.7. Let $\Omega$ and $L$ be as in Theorem 7.4. Let $1<q<\infty$ and let $\omega \in$ $M_{+}(\Omega)$. Then the following statements are equivalent.

(1) The Dirichlet problem (7.25) has a solution for some $\epsilon>0$.

(2) $G \omega<\infty$ and there exists a constant $C>0$ such that

$$
G\left(G^{\omega} \mathbf{1}\right)^{q} \leq C G^{\omega} \mathbf{1}
$$

(3) The weighted inequality

$$
\int_{\Omega}(G h)^{p} \delta^{1-p} d \omega \leq C \int_{\Omega} h^{p} \delta^{1-p} d \sigma
$$

holds.

(4) The testing inequality

$$
\int_{B \cap \Omega}\left(G^{\omega} \chi_{B}\right)^{q} \delta d x \leq C \int_{B} \delta d \omega
$$

holds for all Euclidean balls $B$.

(5) There exists a constant $C$ such that

$$
\int_{E} \delta(x) d \omega(x) \leq C \operatorname{Cap}_{p, G}(E)
$$

for all compact sets $E \subset \Omega$.

Remarks. (a) The equivalence of (1) and (5) for compactly supported $\omega$ was proven in [3]. As in our preceding results, (7.28) with $C=p^{1-q} q^{-1}$ implies that equation (7.25) with $\epsilon=1$ has a solution $u$ such that $G \omega \leq u \leq p G \omega$.

(b) Theorem 7.7 together with the estimate of solution given above yields the following criterion for the existence of solutions in $L^{r}, 0<r \leq \infty$ (or any other ideal function space): (7.25) has a solution for some $\epsilon>0$ which belongs to $L^{r}$ if and only if (7.28) holds and $G^{\omega} \mathbf{1} \in L^{r}$. 
Proof. The theorem follows from Theorem 7.6 if one can show that the corresponding infinitesimal inequality is a consequence of the testing inequality. To prove this, we apply Theorem 5.9 to the integral operator with the Naïm kernel and then pass to the Green potential as above.

Recall that the Naïm kernel $N(x, y)$ is quasi-metric and

$$
\rho(x, y)=N(x, y)^{-1} \asymp|x-y|^{n-2} \max \{|x-y|, \delta(x), \delta(y)\}^{2}
$$

(see the proof of Lemma 7.1). It only remains to show that the assumptions of Theorem 5.9 hold (see also the remark after Theorem 5.9), namely that

$$
\left|B_{2 a}(x)\right|_{\sigma} \leq C a M(x, a)
$$

and

$$
M(y, a) \leq C M(x, a)
$$

whenever $x, y \in X$ and $\rho(x, y) \leq a$. Here

$$
B_{a}(x)=\left\{y \in \Omega: \rho(x, y)<a, d \sigma=\delta(x)^{1+q} d x\right\}
$$

and

$$
M(x, a)=\int_{0}^{a} \frac{\left|B_{t}(x)\right|_{\sigma}}{t^{2}} d t .
$$

Denote Euclidean balls by $\mathcal{B}=\mathcal{B}_{r}(x)$. Note that if we set

$$
\alpha=a^{1 / n}, \quad \beta=a^{1 /(n-2)} \delta(x)^{-2 /(n-2)},
$$

then $B_{a}(x) \subset \mathcal{B}_{\min (\alpha, \beta)}(x)$. On the other hand if $|x-y| \leq r$, then $\rho(x, y) \leq$ $4 \max \left\{r^{n}, r^{n-2} \delta(x)^{2}\right\}$. Hence $\mathcal{B}_{4^{1 / n} \min (\alpha, \beta)}(x) \subset B_{a}(x)$.

It is easy to see that if $a<C \delta(x)^{n}$, this leads to an estimate

$$
\left|B_{a}(x)\right|_{\sigma} \sim \delta(x)^{1+q} \beta^{n}=a^{n /(n-2)} \delta(x)^{1+q-2 n /(n-2)} .
$$

Let $\delta_{0}$ be such that if $\delta(x)+r<\delta_{0}$, then there exists $y \in \mathcal{B}_{r}(x)$ with $\delta(x)=\delta(y)+r$. If $a<2^{-n} \delta_{0}^{n}$ and $2 \delta<a^{1 / n}$, then pick $y \in \mathcal{B}_{\alpha / 2}(x)$ with $\delta(y) \geq \alpha / 2$. Then $\mathcal{B}_{\alpha / 2}(y) \subset B_{a}(x) \subset \mathcal{B}_{3 \alpha / 2}(y)$. From this we get an estimate:

$$
\left|B_{a}(x)\right|_{\sigma} \sim \alpha^{1+q} \alpha^{n}=a^{1+(1+q) / n} .
$$

Combining these estimates we have

$$
\left|B_{a}(x)\right|_{\sigma} \sim \max \left\{a^{n /(n-2)} \delta(x)^{1+q-2 n /(n-2)}, a^{1+(1+q) / n}\right\}
$$

for small enough $a<c \delta_{0}^{n}$. Since $\left|B_{a}(x)\right|_{\sigma}$ is bounded for $a \geq \delta_{0}$, now it is easy to verify that (7.30) and (7.31) hold.

Addendum. After this paper was accepted for publication we learned that $\mathrm{H}$. Brezis and X. Cabre have been able to modify their approach in [9] (see the Introduction) to find another proof of the necessity of our condition $G\left(\sigma(G \omega)^{q}\right) \leq C G \omega$ for the solvability of the Dirichlet problem for $-\Delta u=\sigma u^{q}+\omega$. Moreover, they showed that the constant $C$ in the necessity part can be chosen as $C=p-1$, which complements our sufficiency result with the sharp constant $C=q^{-1} p^{1-q}$. Note that the latter constant is applicable to very general superlinear operator equations, while the former is established only for the Laplacian. For more general superlinear differential and integral equations the constant $C=C(q, \kappa)$ in the necessity statements could be easily estimated from our proof; generally it depends on the quasi-metric constant of the kernel. 
Added in proof. We have recently learned that Lemma 7.1 (in a different but equivalent form) was found earlier by M. Selmi, Comparaison des noyaux de Green sur les domaines $C^{1,1}$, Rev. Roumaine Math. Pures Appl 36 (1991), 91-100. MR 92k:35048

\section{REFERENCES}

1. D. R. Adams, Weighted nonlinear potential theory, Trans. Amer. Math. Soc. 297 (1986), 73-94. MR 88m:31011

2. D. R. Adams and L. I. Hedberg, Function Spaces and Potential Theory, Springer-Verlag, Berlin-Heidelberg-New York, 1996. MR 97j:46024

3. D. R. Adams and M. Pierre, Capacitary strong type estimates in semilinear problems, Ann. Inst. Fourier, Grenoble 41 (1991), 117-135. MR 92m:35074

4. H. Aikawa and M. Essén, Topics in Potential Theory, Lecture Notes, Uppsala Univ., no. 1, 1994.

5. A. Ancona, Comparison of Green's functions for elliptic operators on manifolds or domains, Preprint 96-11, Université de Paris-Sud, 1996, pp. 1-42.

6. P. Baras and M. Pierre, Critère d'existence de solutions positives pour des équations semilinéaires non monotones, Ann. Inst. H. Poincaré, Analyse Non Linéaire 2 (1985), 185-212. MR 87j: 45032

7. R. F. Bass, Probabilistic Techniques in Analysis, Springer-Verlag, Berlin-Heidelberg-New York, 1995. MR 96e:60001

8. H. Brezis and L. Nirenberg, Positive solutions of nonlinear equations involving critical Sobolev exponents, Comm. Pure. Appl. Math. 36 (1983), 437-477. MR 84h:35059

9. H. Brezis and X. Cabre, Some simple nonlinear PDE's without solutions, preprint (1997), 1-37; Boll. Unione Mat. Ital. Sez. B Artic. Ric. Mat. (8) 1 (1998), 223-262.

10. L. Carleson, Selected Problems on Exceptional Sets, Van Nostrand Math. Studies, no. 13, Van Nostrand, Princeton, 1967. MR 37:1576

11. R. Coifman and G. Weiss, Extensions of Hardy spaces and their use in analysis, Bull. Amer. Math. Soc. 83 (1977), 569-645. MR 56:6264

12. M. Cranston, E. Fabes and Z. Zhao, Conditional gauge and potential theory for the Schrödinger operator, Trans. Amer. Math. Soc. 307 (1988), 171-194. MR 90a:60135

13. K. L. Chung and Z. Zhao, From Brownian motion to Schrödinger's equation, Springer-Verlag, Berlin-Heidelberg-New York, 1995. MR 96f:60140

14. E. B. Dynkin, Superprocesses and partial differential equations, Ann. Probab. 21 (1993), 1185-1262. MR 94j:60156

15. E. B. Dynkin and S. E. Kuznetsov, Superdiffusions and removable singularities for quasilinear partial differential equations, Comm. Pure Appl. Math. 49 (1996), 125-176. MR 97m:60114

16. C. Fefferman, The uncertainty principle, Bull. Amer. Math. Soc. 9 (1983), 129-206.

17. M. Frazier, B. Jawerth and G. Weiss, Littlewood-Paley Theory and the Study of Function Spaces, CBMS-AMS Regional Conf. Series, vol. 79, 1991. MR 92m:42021

18. J. Garcia-Cuerva and J.-L. Rubio de Francia, Weighted Norm Inequalities and Related Topics, North-Holland Math. Studies, vol. 116, North-Holland, Amsterdam, 1985. MR 87d:42023

19. J. Garnett, Bounded Analytic Functions, Academic Press, New York-London-Toronto, 1981.

20. A. E. Gatto, C. Segovia and S. Vági, On fractional differentiation on spaces of homogeneous type, Revista Mat. Iberoamer. 12 (1996), 1-35. MR 97k:42028

21. K. Hansson, Imbedding theorems of Sobolev type in potential theory, Math. Scand. 45 (1979), 77-102. MR 81j:31007

22. K. Hansson, V. G. Maz'ya and I. E. Verbitsky, Criteria of solvability for multidimensional Riccati's equations, to appear in Arkiv för Matem.

23. L. I. Hedberg and T. Wolff, Thin sets in nonlinear potential theory, Ann. Inst. Fourier (Grenoble) 33 (1983), 161-187. MR 85f:31015

24. J. Heinonen, T. Kilpeläinen and O. Martio, Nonlinear Potential Theory of Degenerate Elliptic Equations, Clarendon Press, Oxford-New York-Tokyo, 1993. MR 94e:31003

25. H. Hueber and M. Sieveking, Uniform bounds for quotients of Green functions on $C^{1,1}$ domains, Ann. Inst. Fourier, Grenoble 32 (1982), 105-117. MR 84a:35063

26. N. J. Kalton and L. Tzafriri, Legendre and Jacobi polynomials in $L_{p}$ spaces and the $\Lambda_{p}$ problem, in preparation. 
27. R. Kerman and E. Sawyer, The trace inequality and eigenvalue estimates for Schrödinger operators, Ann. Inst. Fourier (Grenoble) 36 (1986), 207-228. MR 88b:35150

28. J. Komlos, A generalization of a problem of Steinhaus, Acta Math. Sci. Hungar. 18 (1967), 217-229. MR 35:1071

29. M. A. Krasnosel'skii and P. P. Zabreiko, Geometrical Methods of Nonlinear Analysis, Springer-Verlag, Berlin-Heidelberg-New York, 1984. MR 85b:47057

30. J.-F. Le Gall, A class of path-valued Markov processes and its applications to superprocesses, Probab. Theory Related Fields 95 (1993), 25-46. MR 94f:60093

31. J. Lindenstrauss and L. Tzafriri, Classical Banach Spaces, II, Springer-Verlag, Berlin-Heidelberg-New York, 1979. MR 81c:46001

32. P. L. Lions, On the existence of positive solutions of semilinear elliptic equations, SIAM Review 24 (1982), 441-467. MR 84a:35093

33. B. Maurey, Théorèmes de factorisation pour les opérateurs linéaires à valeurs dans un espaces $L^{p}$, Astérisque, vol. 11, Soc. Math. de France, Paris, 1974. MR 49:9670

34. V. G. Maz'ya, On the theory of the n-dimensional Schrödinger operator, Izv. Akad. Nauk SSSR, Ser. Matem. 28 (1964), 1145-1172.

35. V. G. Maz'ya, Sobolev Spaces, Springer-Verlag, Berlin-Heidelberg-New York, $1985 . \quad$ MR 87g:46056

36. V. G. Maz'ya, Beurling's theorem on a minimum principle for positive harmonic functions, Zapiski Nauchn. Sem. LOMI 30 (1972), 76-90; English transl. in. J. Soviet Math. 4 (1972), 367-379.

37. V. G. Maz'ya and I. E. Verbitsky, Capacitary estimates for fractional integrals, with applications to partial differential equations and Sobolev multipliers, Arkiv för Matem. 33 (1995), 81-115. MR 96i: 26021

38. L. Naïm, Sur le rôle de la frontière de R. S. Martin dans la théorie du potentiel, Ann. Inst. Fourier, Grenoble 7 (1957), 183-281. MR 20:6608

39. E. M. Nikishin, Resonance theorems and superlinear operators, Russian Math. Surveys 25 (1970), 124-187.

40. N. K. Nikolskii, Treatise on the Shift Operator, Springer-Verlag, Berlin-Heidelberg-New York, 1986. MR 87i:47042

41. C. Pérez, Two weighted norm inequalities for Riesz potentials and uniform $L_{p}$-weighted Sobolev inequalities, Indiana Univ. Math. J. 39 (1990), 31-44. MR 92a:42024

42. J. L. Rubio de Francia, Weighted norm inequalities and vector valued inequalities, Harmonic Analysis, Proc., Minneapolis, 1981; , Lecture Notes in Math. 908 (1982), 86-101. MR 83h: 42025

43. E. T. Sawyer, Two weight norm inequalities for fractional and Poisson integrals, Harmonic Analysis, Proc., Minneapolis, 1981; Lecture Notes in Math. 908 (1982), 102-127. MR 83k:42020b

44. E. T. Sawyer, Two weight norm inequalities for certain maximal and integral operators, Trans. Amer. Math. Soc. 308 (1988), 533-545. MR 89d:26009

45. E. T. Sawyer and R. L. Wheeden, Weighted inequalities for fractional integrals on Euclidean and homogeneous spaces, Amer. J. Math. 114 (1992), 813-874. MR 94i:42024

46. E. T. Sawyer, R. L. Wheeden and S. Zhao, Weighted norm inequalities for operators of potential type and fractional maximal functions, Potential Analysis 5 (1996), 523-580. MR 98g:42026

47. M. Schechter, Superlinear elliptic boundary value problems, Manuscripta Math. 86 (1995), 253-267. MR 96e:35052

48. J. Serrin, On the Harnack inequality for linear elliptic equations, J. d'Analyse Math. 4 (1956), 292-308. MR 18:398f

49. G. Sweers, Positivity for a strongly coupled elliptic system, J. Geom. Anal. 4 (1994), 121-142. MR 95g:35052

50. S. Treil and A. Volberg, Weighted imbedding and weighted norm inequalities for the Hilbert transform and maximal operators, St. Petersburg Math. J. 7 (1996), 1017-1032. MR 97c: 42017

51. B. O. Turesson, Nonlinear Potential Theory and Weighted Sobolev Spaces, Linköping Studies in Sci. and Techn., Dissert. 387, Linköping University, 1995.

52. I. E. Verbitsky and R. L. Wheeden, Weighted inequalities for fractional integrals and applications to semilinear equations, J. Funct. Anal. 129 (1995), 221-241. MR 95m:42025 
53. I. E. Verbitsky and R. L. Wheeden, Weighted norm inequalities for integral operators, Trans. Amer. Math. Soc. 350 (1998), 3371-3391. MR 98k:42020

54. R. L. Wheeden and S. Zhao, Weak type estimates for operators of potential type, Studia Math. 119 (1996), 149-160. MR 97d:42013

55. K.-O. Widman, Inequalities for the Green function and boundary continuity of the gradients of solutions of elliptic differential equations, Math. Scand. 21 (1967), 13-67. MR 39:621

56. Z. Zhao, Green function for Schrödinger operator and conditioned Feynman-Kac gauge, J. Math. Anal. Appl. 116 (1986), 309-334. MR 88f:60142

57. W. P. Ziemer, Weakly Differentiable Functions, Springer-Verlag, New York, 1989. MR 91e:46046

Department of Mathematics, University of Missouri, Columbia, Missouri 65211

E-mail address: nigel@math.missouri.edu

Department of Mathematics, University of Missouri, Columbia, Missouri 65211

E-mail address: igor@math.missouri.edu 